ACCepted For publication in the Astrophysical Journal.

Preprint typeset using $\mathrm{LATEX}_{\mathrm{E}}$ style emulateapj v. 08/22/09

\title{
ABUNDANCE PROFILING OF EXTREMELY METAL-POOR STARS AND SUPERNOVA PROPERTIES IN THE EARLY UNIVERSE
}

\author{
Nozomu Tominaga ${ }^{1,2}$, Nobuyuki Iwamoto ${ }^{3}$, Ken' $^{\prime}$ ichi Nomoto ${ }^{2,4}$ \\ Accepted for publication in the Astrophysical Journal.
}

\begin{abstract}
After the Big Bang nucleosynthesis, the first heavy element enrichment in the Universe was made by a supernova (SN) explosion of a population (Pop) III star (Pop III SN). The abundance ratios of elements produced from Pop III SNe are recorded in abundance patterns of extremely metal-poor (EMP) stars. The observations of the increasing number of EMP stars have made it possible to statistically constrain the explosion properties of Pop III SNe. We present Pop III SN models whose nucleosynthesis yields well-reproduce individually the abundance patterns of 48 such metal-poor stars as $[\mathrm{Fe} / \mathrm{H}] \lesssim-3.5$. We then derive relations between the abundance ratios of EMP stars and certain explosion properties of Pop III SNe: the higher $[(\mathrm{C}+\mathrm{N}) / \mathrm{Fe}]$ and $[(\mathrm{C}+\mathrm{N}) / \mathrm{Mg}]$ ratios correspond to the smaller ejected $\mathrm{Fe}$ mass and the larger compact remnant mass, respectively. Using these relations, the distributions of the abundance ratios of EMP stars are converted to those of the explosion properties of Pop III SNe. Such distributions are compared with those of the explosion properties of present day SNe: The distribution of the ejected Fe mass of Pop III SNe has the same peak as that of the present day SNe but shows an extended tail down to $\sim 10^{-2}-10^{-5} \mathrm{M}_{\odot}$, and the distribution of the mass of the compact remnant of Pop III SNe is as wide as that of the present day stellar-mass black holes. Our results demonstrate the importance of large samples of EMP stars obtained by ongoing and future EMP star surveys and subsequent high-dispersion spectroscopic observations in clarifying the nature of Pop III SNe in the early Universe.

Subject headings: Galaxy: halo - nuclear reactions, nucleosynthesis, abundances — stars: abundances — stars: Population III — supernovae: general
\end{abstract}

\section{INTRODUCTION}

Just after the Big Bang, the Universe was composed of only $\mathrm{H}, \mathrm{He}$, and a small amount of light elements and its further chemical enrichment was provided by stars and supernovae (SNe). The early history of the chemical enrichment is recorded in chemical abundance of longlived low-mass stars that were formed in the early Universe. Such old stars show low heavy element (metal) abundances, thus being called metal-poor (MP) stars. A large number of candidates of MP stars have been discovered by several large survey programs (e.g., HK survey: Beers et al. 1992, 1999; Hamburg/ESO survey: Christlieb 2003; SEGUE survey: Yanny et al. 2009). High-dispersion spectroscopic observations of these candidates have been made to accurately determine their metallicities and detailed elemental abundances (e.g., Rvan et al. 1996, see also Yong et al. 2013 and the series for recent reviews).

The first enrichment of heavy elements in the Universe was provided by supernova (SN) explosions of population (Pop) III stars (Pop III SNe), which ejected synthesized metals. The next-generation stars formed from gases of

\footnotetext{
${ }^{1}$ Department of Physics, Faculty of Science and Engineering, Konan University, 8-9-1 Okamoto, Kobe, Hyogo 658-8501, Japan; tominaga@konan-u.ac.jp

2 Kavli Institute for the Physics and Mathematics of the Universe (WPI), The University of Tokyo, 5-1-5 Kashiwanoha, Kashiwa, Chiba 277-8583, Japan

${ }^{3}$ Nuclear Data Center, Japan Atomic Energy Agency, Tokai, Ibaraki 319-1195, Japan; iwamoto.nobuyuki@jaea.go.jp

${ }^{4}$ Department of Astronomy, School of Science, The University of Tokyo, Bunkyo-ku, Tokyo 113-0033, Japan; nomoto@astron.s.utokyo.ac.jp
}

mixture of the SN ejecta and pristine interstellar matter. Metal abundance ratios in those second-generation stars directly reflect nucleosynthesis in the Pop III SNe. The ejecta of subsequent $\mathrm{SNe}$ overlapped with each other, from which later-generation stars formed. After many cycles of such star formation and SN explosions, the heavy elements from numerous SN ejecta were wellmixed in the interstellar gas and, eventually, the interstellar medium (ISM) became chemically homogeneous. In the Galactic halo, such a transition from the chemically inhomogeneous to homogeneous ISM took place at $[\mathrm{Fe} / \mathrm{H}]^{5} \sim-3$ to -2.5 as suggested from the changes in the abundance patterns of the MP stars, especially $r$ process elements, and the hierarchical chemical evolution models (e.g., Argast et al. 2000; Tumlinson 2006).

In the inhomogeneous environment, chemical enrichment by a single SN dominates the preexisting metal contents (e.g., Audouze \& Silk 1995) and $[\mathrm{Fe} / \mathrm{H}]$ of an MP star is not a time indicator but merely reflects $[\mathrm{Fe} / \mathrm{H}]$ of a cloud in which the MP star formed. A single SN enriches the ISM roughly up to $[\mathrm{Fe} / \mathrm{H}] \sim-3$ (Tominaga et al. 2007b; Nomoto et al. 2013). Thus, MP stars with $[\mathrm{Fe} / \mathrm{H}]<-3$, called extremely metal-poor (EMP) stars (Beers \& Christlieb 2005), are regarded to possess nucleosynthetic products of a few Pop III SNe. Inversely, comparisons between nucleosynthetic yields of Pop III SNe and the abundance patterns of the EMP stars constrain explosion properties of Pop III SNe ("abundance profiling").

\footnotetext{
${ }^{5}$ Here $[\mathrm{A} / \mathrm{B}]=\log _{10}\left(N_{\mathrm{A}} / N_{\mathrm{B}}\right)-\log _{10}\left(N_{\mathrm{A}} / N_{\mathrm{B}}\right) \odot$, where the subscript $\odot$ refers to the solar value and $N_{\mathrm{A}}$ and $N_{\mathrm{B}}$ are abundances of elements $\mathrm{A}$ and $\mathrm{B}$, respectively.
} 
Asphericity and the explosion energy of Pop III SNe are particularly important indicators obtained from such comparisons. To consistently reproduce $[\alpha / \mathrm{Fe}]$ and $[(\mathrm{Co}, \mathrm{Zn}) / \mathrm{Fe}]$ of EMP stars, materials synthesized in the deep complete Si-burning region (including Co and $\mathrm{Zn}$ ) should be ejected but the amount of Fe should not be too large relative to $\alpha$ elements. This requirement is satisfied with taking into account aspherical effects such as the jet-like explosion (Tominaga et al. 2007a; Tominaga 2009) or the Rayleigh-Taylor instability (Joggerst et al. 2009, 2010). The aspherical explosion is mimicked by the mixing-and-fallback model that wellreproduces the abundance patterns of the EMP stars (Umeda \& Nomoto 2002, 2003, 2005; Iwamoto et al. 2005; Tominaga et al. 2007b; Heger \& Wooslev 2010). Furthermore, the trends of increasing $[(\mathrm{Co}, \mathrm{Zn}) / \mathrm{Fe}]$ toward lower $[\mathrm{Fe} / \mathrm{H}]$ is shown to be explained if less-massive stars (with main-sequence mass $M_{\mathrm{MS}} \lesssim 20 \mathrm{M}_{\odot}$ ) produce normal SNe and more-massive stars (with $M_{\mathrm{MS}} \gtrsim$ $\left.20 \mathrm{M}_{\odot}\right)$ produce energetic explosions, i.e., hypernovae (Umeda \& Nomoto 2005; Tominaga et al. 2007b).

The asphericity and the dependence of explosion energy $(E)$ on $M_{\mathrm{MS}}$ are also seen in the nearby present day SNe. Their nebular spectra and polarization indicate aspherical distributions of elements (e.g., Maeda et al. 2008; Tanaka et al. 2012). And $M_{\mathrm{MS}}$ and $E$ of the present day SNe are constrained from their light curves and spectra (e.g., Nomoto et al. 2006). The above variations of the present day $\mathrm{SNe}$ are consistent with the requirements for the variations of Pop III SNe to reproduce the abundance patterns and the trends of EMP stars. Such a consistency suggests that the SN explosion mechanism may not be so sensitive to the metallicity.

However, Pop III SN models for individual EMP stars have been so far constructed only for a few EMP stars which have interesting characteristics (e.g., Umeda \& Nomoto 2003; Iwamoto et al. 2005). Also, because of the lack of large samples of EMP stars, it has not been possible to investigate the statistical properties of Pop III SNe. Recently the number of the EMP stars increased dramatically as a result of the large MP star surveys, intensive follow-up high-dispersion spectroscopic observations, and the development of stellar abundance analysis methods. Therefore, it has become feasible and necessary to statistically constrain the explosion properties of Pop III SNe using the EMP stars.

In this paper, we focus on the 48 most metal-poor stars $^{6}$ with $[\mathrm{Fe} / \mathrm{H}] \lesssim-3.5$ including 41 halo stars and 7 stars in dwarf galaxies, and construct Pop III SN models to reproduce their abundance patterns. With reasonably successful models, the abundance ratios of these EMP stars can be directly related to the explosion properties of Pop III SNe: the masses of ejected Fe $M_{\mathrm{ej}}(\mathrm{Fe})$ and the compact remnant $M_{\text {rem }}$. According to these relations, we derive the distributions of $M_{\mathrm{ej}}(\mathrm{Fe})$ and $M_{\mathrm{rem}}$ of Pop III SNe. These relations make it possible to extract infor-

6 The stars are found in ADS (http://adsww.harvard.edu/), SAGA database (http://saga.sci.hokudai.ac.jp/ Suda et al. 2008, 2011), and a comprehensive study on the EMP stars (Yong et al.|2013). We except the Si-deficient star, HE 1424-0241 (Cohen et al. 2007), because its abundance pattern can be reproduced only with an aspherical abundance distribution of the jet-like explosion (Tominaga 2009). mation of Pop III SNe without constructing SN models at the era that large samples of EMP stars are available.

We describe the model and procedure in $\S 2$ and present SN models in $\S 3$. After discussing the limitations of our approach in $\S$ 4, the distributions of properties of Pop III SNe are presented in $\S 5$. The paper is concluded with several discussion in $\S[$ and remarks in $\S[$.

\section{MODEL \& METHOD}

We adopt the $25 \mathrm{M}_{\odot}$ progenitor models to construct Pop III SN models for all EMP stars in a unified manner, and the $40 \mathrm{M}_{\odot}$ progenitor model to investigate dependence on $M_{\mathrm{MS}}$. The $25 \mathrm{M}_{\odot}$ progenitor models with a different mixing efficiency are taken from Iwamoto et al. $(2005)$ and the $40 \mathrm{M}_{\odot}$ progenitor model is taken from Tominaga et al. (2007a) as follows:

(1) Model $\mathrm{A}$ is the $25 \mathrm{M}_{\odot}$ progenitor without enhanced mixing,

(2) Model B is the $25 \mathrm{M}_{\odot}$ progenitor with enhanced mixing, e.g., due to rapid rotation,

and

(3) Model $\mathrm{C}$ is the $40 \mathrm{M}_{\odot}$ progenitor without enhanced mixing.

The difference due to the enhanced mixing prominently appears in the abundance of $\mathrm{N}$ that is synthesized via the $\mathrm{CNO}$ cycle facilitated by the mixing of $\mathrm{C}$ in the $\mathrm{He}$ convective shell into the H-rich envelope. Thus Models $\mathrm{A}$ and $\mathrm{C}$ have a low $\mathrm{N}$ abundance, while Model B has a high $\mathrm{N}$ abundance in the $\mathrm{H}$ layer (Figs. 1 $1 \mathrm{a}-1 \mathrm{k}$ ). The efficient mixing also considerably enhances the He abundance in the $\mathrm{H}$ layer. The progenitor models are summarized in Table 1. We adopt explosion energies $E_{51}=E /\left(10^{51} \mathrm{erg}\right)=0.72,1,3,5,10$, and 20 for Models $\mathrm{A}$ and $\mathrm{B}$, and $E_{51}=10,15,20$, and 30 for Model C.

Hydrodynamical and nucleosynthesis calculations are performed with the same method as in Tominaga et al. $(2007 \mathrm{~b})$. The explosion is initiated by a thermal bomb and followed with the Lagrangian code assuming spherical symmetry. The hydrodynamical calculation includes nuclear energy releases from the $\alpha$-network and a realistic equation of state (Nomoto 1982; Nomoto \& Hashimoto 1988). The detailed nucleosynthesis calculation is performed as a post-processing (Hix \& Thielemann 1996, 1999) with a reaction network including 280 isotopes up to ${ }^{79} \mathrm{Br}$ (Table 1 in Umeda \& Nomoto 2005).

In order to take into account the aspherical effect, we apply the mixing-and-fallback model to the spherical calculations. The mixing-and-fallback model has three parameters characterizing the asphericity: the initial mass cut $M_{\text {cut }}$, the outer boundary of the mixing region $M_{\text {mix }}$, and the ejection factor $f_{\mathrm{ej}}$ (Appendix in Tominaga et al. $2007 \mathrm{~b})$. The mass of the compact remnant $M_{\text {rem }}$ is derived as

$$
M_{\text {rem }}=M_{\text {cut }}+\left(1-f_{\mathrm{ej}}\right)\left(M_{\mathrm{mix}}-M_{\mathrm{cut}}\right) .
$$

In the jet-induced explosion model, physical meanings of $M_{\text {cut }}, f_{\text {ej }}$, and $M_{\text {rem }}$ are the inner boundary, the fraction of the solid angle of a region from which materials produced by incomplete Si burning are ejected, and the remnant mass, respectively (Tominaga|2009). An explosion with weak jets, i.e., with low energy deposition rate, 


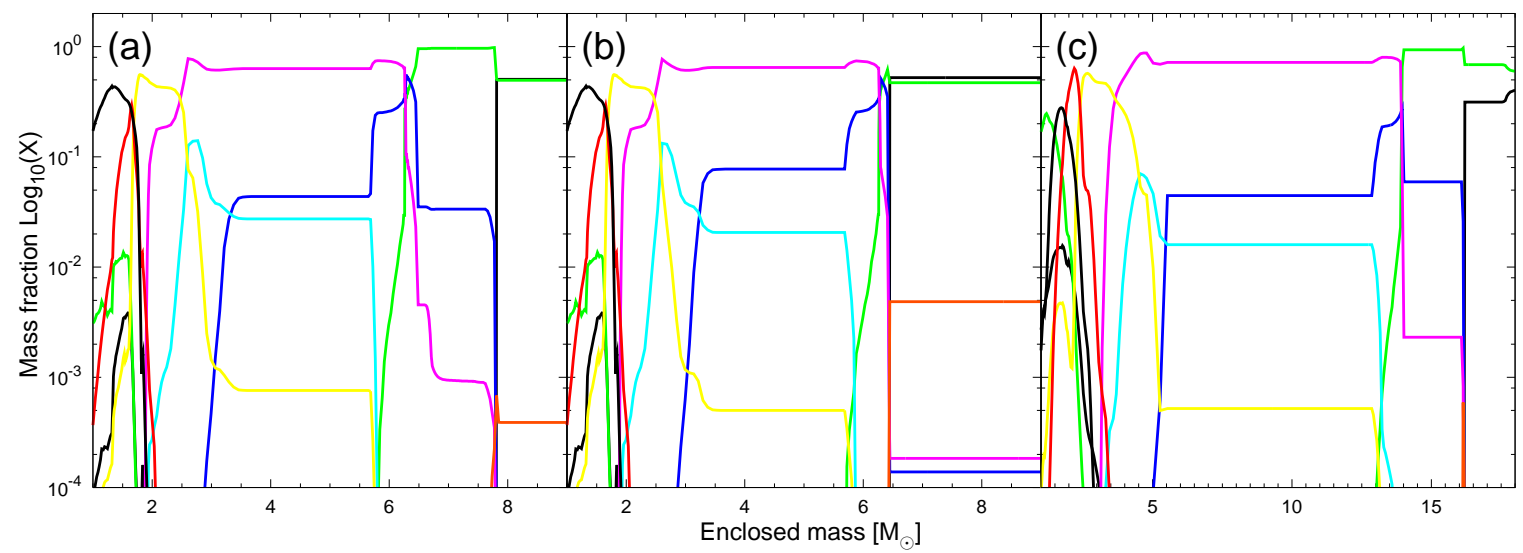

FIG. 1.- Abundance distribution against the enclosed mass $M_{r}$ before the explosion of (a) 25M $\odot$ star without mixing enhancement (Model A), (b) $25 \mathrm{M}_{\odot}$ star with mixing enhancement (Model B), and (c) $40 \mathrm{M}_{\odot}$ star without mixing enhancement (Model C). The colors represent ${ }^{1} \mathrm{H}$ (black), ${ }^{4} \mathrm{He}$ (green), ${ }^{12} \mathrm{C}$ (blue), ${ }^{14} \mathrm{~N}$ (orange), ${ }^{16} \mathrm{O}$ (magenta), ${ }^{24} \mathrm{Mg}$ (cyan), ${ }^{28} \mathrm{Si}$ (yellow), ${ }^{54} \mathrm{Fe}\left(\right.$ gray), and ${ }^{56} \mathrm{Ni}($ red).

corresponds to an $\mathrm{SN}$ model with small $f_{\mathrm{ej}}$ and large $M_{\text {rem }}$ (Tominaga et al. 2007a).

Tominaga (2009) showed that the mixing-and-fallback model do not fully reproduce the enhanced abundance ratios $[(\mathrm{Sc}, \mathrm{Ti}, \mathrm{Co}, \mathrm{Zn}) / \mathrm{Fe}]$ obtained in the jet-induced explosion model. The enhancement stems from nucleosynthesis in a high-entropy environment which is realized by the concentration of energy in the narrow jet. Thus, we adopt "low-density" modification as in Tominaga et al. (2007b) to mimic the high-entropy environment, in which the density of the progenitor is artificially reduced by a factor of "low-density" factor $f_{\rho}$ without changing the mass of each Lagrangian mass shell. Furthermore, since the neutrino absorption during the explosion may largely change the $Y_{\mathrm{e}}$ distribution in the Si burning layers (e.g., Janka et al. 2012; Kotake et al. 2012; Burrows 2013; Bruenn et al. 2013, for recent review), which is not included in our explosion calculations, we modify $Y_{\mathrm{e}}$ profile to $Y_{\mathrm{e}}=0.5001$ in the complete Si burning region if $[\mathrm{Co} / \mathrm{Fe}]$ of an $\mathrm{SN}$ model is underabundant and/or $Y_{\mathrm{e}}=0.4997$ in the incomplete Si burning region if $[\mathrm{Mn} / \mathrm{Fe}]$ of an SN model is underabundant (Umeda \& Nomoto 2005). Because the explosion models with realistic geometry and the neutrino processes have not been established, these modifications to compare with the observed abundance ratios of many EMP stars may provide useful constraints on the entropy and $Y_{\mathrm{e}}$ distribution in the realistic explosion models. We note that these two modifications do not affect on the distribution of $M_{\mathrm{ej}}(\mathrm{Fe})$ and $M_{\text {rem }}$ of Pop III SNe ( $\S 5$.

\section{COMPARISON WITH THE OBSERVATIONS}

Hydrodynamical and thermodynamical features of SNe appear in abundance ratios between elements synthesized in different layers and between elements synthesized in the same layer, respectively. It is important to determine abundances of elements as many as possible because different abundance ratios provide different information. We focus on the hydrodynamical features in this paper and select the 48 EMP stars (including a binary) which have at least 3 determined abundance ratios to Fe of the following elements: (1) C, N, or O, (2) Mg, (3) Ca, and (4) Co or Ni. They consist of 19 carbonnormal and nitrogen-normal EMP stars including a binary system, 12 carbon-enhanced metal-poor (CEMP) stars, 6 nitrogen-enhanced metal-poor (NEMP) stars, 2 ultra metal-poor (UMP) star, and 2 hyper metal-poor (HMP) stars in Galactic halo and 7 EMP stars in dwarf galaxies. Definitions of classification are described in respective subsections. The stars and references are summarized in Table 2.

Since the theoretical model provides mass fractions of elements and isotopes, the theoretical SN yields should be compared with genuine abundance ratios taking into account non-LTE (NLTE) and 3D effects. However, the NLTE or 3D effect has been estimated only for several MP stars (e.g., Collet et al. 2006; Andrievsky et al. 2007, 2008, 2010) and 3D-NLTE abundance ratios are currently not available for any stars. Results could be systematically biased if we adopt 1D-LTE abundance ratios. On the other hand, in order to investigate statistical nature of Pop III SNe, it is requested to avoid including artificial scatter by adopting different stellar atmosphere models, i.e., to derive abundance ratios with a uniform method. Yong et al. (2013) recently present homologous abundance analyses and discuss distribution of abundance ratios but this study is restricted within a framework of a 1D-LTE assumption.

In this paper, we adopt abundance ratios in Yong et al. (2013) and in other literatures which may take into account NLTE or 3D effect (Table 2). The abundance patterns of the EMP stars and the SN yields are normalized with the solar abundance ratios in Asplund et al. (2009). If there are plural references, we adopt their average $[\mathrm{Fe} / \mathrm{H}]$. We construct an SN model to reproduce NLTE or 3D corrected abundance ratios if the corrections specified for the individual EMP star are available. If not, we construct an SN model to reproduce 1D-LTE abundance ratios but also refer abundance ratios for which the following NLTE correction is expediently applied:

- $\log (g)$ and $[\mathrm{Fe} / \mathrm{H}]$-dependent correction for $[\mathrm{Na} / \mathrm{Fe}]$ (Andrievsky et al. 2007, see also Lind et al. 2011),

- +0.2 for $[\mathrm{Mg} / \mathrm{Fe}]$ (Andrievsky et al. 2010),

- $T_{\text {eff }}, \log (g)$, and $[\mathrm{Fe} / \mathrm{H}]$-dependent correction for [Al/Fe] (Andrievsky et al. 2008),

- -0.2 for $[\mathrm{K} / \mathrm{Fe}]$ (Takeda et al. 2009; Andrievsky et al. 2010), 
- +0.4 for $[\mathrm{Cr} / \mathrm{Fe}]$ derived from $\mathrm{Cr}$ I line(s) (Bergemann \& Cescutti 2010), and

- +0.6 for $[\mathrm{Mn} / \mathrm{Fe}]$ derived from $\mathrm{Mn}$ I resonance line(s) (Bergemann \& Gehren 2008).

The corrections for $[\mathrm{Na} / \mathrm{Fe}]$ and $[\mathrm{Al} / \mathrm{Fe}]$ are estimated from the interpolation and extrapolation of the corrections given by in Andrievsky et al. (2007, 2008), and the average corrections are uniformly adopted for other elements. The true correction depends on the stellar parameters and should be estimated for the individual star. Thus, the range between 1D-LTE and expedientlycorrected ratios is taken as a range of uncertainty.

The properties of Pop III SN models are summarized in Table 3. The comparisons between the abundance patterns of the EMP stars and the SN yields are shown in Figures $2 \mathrm{a}-2 \mathrm{a}$ and $3 \mathrm{a}-3 \mathrm{~h}$ for the carbon-normal and nitrogen-normal EMP stars, Figures $4 \mathrm{a}-4$ for the CEMP stars, Figures $5 \mathrm{a}-5 \mathrm{f}$ for the NEMP stars, Figures $6 \mathrm{a}-6 \mathrm{~b}$ for the UMP stars, Figures $7 \mathrm{a}-7 \mathrm{~b}$ for the HMP stars, and Figures $8 \mathrm{a}-8 \mathrm{~b}$, for the EMP stars in dwarf galaxies.

\subsection{Extremely Metal-Poor stars}

In this subsection, we examine 19 carbon-normal and nitrogen-normal EMP stars with $-4 \lesssim[\mathrm{Fe} / \mathrm{H}] \lesssim-3.5$.

HE 0146-1548: The metallicity is $[\mathrm{Fe} / \mathrm{H}]=-3.46$ and the abundance ratios are shown in Figure $2 \mathrm{a}$ (Yong et al. 2013; Norris et al. 2013). The abundance pattern is well reproduced by an SN model with $E_{51}=5$, $M_{\text {rem }}=3.45 \mathrm{M}_{\odot}$, and $M_{\text {ej }}(\mathrm{Fe})=2.38 \times 10^{-2} \mathrm{M}_{\odot}$.

$\star[\mathrm{Na} / \mathrm{Mg}]:$ The high $[\mathrm{Na} / \mathrm{Mg}](>0)$ is realized by the mixing region extending to the middle of the $\mathrm{O}+\mathrm{Mg}$ layer above which Na synthesized by static burning is not destroyed by explosive burning.

$\star[(\mathbf{T i}, \mathbf{C o}) / \mathbf{F e}]$ : The high $[(\mathrm{Ti}, \mathrm{Co}) / \mathrm{Fe}]$ indicates that explosive nucleosynthesis takes place in the high-entropy environment.

CS 22189-009: The metallicity is $[\mathrm{Fe} / \mathrm{H}]=$ -3.48 and the abundance ratios are shown in Figure 2b (Cavrel et al. 2004; Spite et al. 2005, 2006; Andrievskv et al. 2007, 2008, 2010; Spite et al. 2012; Yong et al. 2013). Since the star shows $[\mathrm{C} / \mathrm{N}] \sim 0$ and the mixing in CS 22189-009 is not strong (Spite et al. 2005, 2006), $\mathrm{N}$ is likely to be synthesized by the CNO cycle in the SN progenitor due to the enhanced mixing. The abundance pattern except for $[\mathrm{K} / \mathrm{Fe}]$ is well reproduced by an SN model with $E_{51}=20, M_{\text {rem }}=4.20 \mathrm{M}_{\odot}$, and $M_{\mathrm{ej}}(\mathrm{Fe})=7.76 \times 10^{-2} \mathrm{M}_{\odot}$.

$\star[\mathbf{K} / \mathbf{F e}]:$ The underproduction of $\mathrm{K}$ could be solved with nucleosynthesis in $p$-rich ejecta which may have been produced by neutrino processes (e.g., Iwamoto et al. 2006). Heger \& Woosley (2010) also presented that $[\mathrm{K} / \mathrm{Fe}]$ could be enhanced in some lessmassive stars.

$\star[\mathbf{F} / \mathbf{F e}]$ : The abundance of $\mathrm{F}$ is enhanced by the hot CNO cycle at the bottom of the $\mathrm{H}$ layer during shock propagation. If an N-rich layer experiences $T \gtrsim 7 \times 10^{8} \mathrm{~K},{ }^{19} \mathrm{~F}$ is synthesized as ${ }^{14} \mathrm{~N}(p, \gamma){ }^{15} \mathrm{O}(\alpha, \gamma){ }^{19} \mathrm{Ne}\left(\beta^{+}\right){ }^{19} \mathrm{~F}$. Such an enhancement of $\mathrm{F}$ results if a progenitor star has the N-rich layer close to the core to experience high enough temperature (see also Chieffi \& Limongi 2012). Thus [F/Fe] could give a clue to constrain $\mathrm{N}$ enhancement in the progenitor ${ }^{7}$ and the explosion energy $(\S 4.2)$.

$\star{ }^{7} \mathbf{L i}$ and ${ }^{11} \mathbf{B}:{ }^{11} \mathrm{~B}$ is synthesized via ${ }^{3} \mathrm{He}(\alpha, \gamma){ }^{7} \mathrm{Be}(\alpha, \gamma){ }^{11} \mathrm{C}\left(\beta^{+}\right){ }^{11} \mathrm{~B}$ in the He-rich layer of the $\mathrm{H}$ envelope which experiences $T \gtrsim 5 \times 10^{8} \mathrm{~K}$. The yield of ${ }^{7} \mathrm{Be}$ decaying to ${ }^{7} \mathrm{Li}$ is $2 \times 10^{-7} \mathrm{M}_{\odot}$, which is smaller than the swept-up mass of primordial ${ }^{7} \mathrm{Li}$ synthesized by big bang nucleosynthesis. ${ }^{8}$

SDSS J090733+024608: The metallicity is $[\mathrm{Fe} / \mathrm{H}]=$ -3.50 . The abundance ratios are presented in Caffau et al. (2011b) but [(C, N, O)/Fe] are not available (Fig. 22). The abundance pattern except for [Ti/Fe] is well reproduced by an $\mathrm{SN}$ model with $E_{51}=5$, $M_{\mathrm{rem}}=1.74 \mathrm{M}_{\odot}$, and $M_{\mathrm{ej}}(\mathrm{Fe})=1.85 \times 10^{-1} \mathrm{M}_{\odot}$. It would be possible to reproduce the abundance pattern without mixing-and-fallback because of no constraint on $[\mathrm{C} / \mathrm{Fe}]$ and low $[\mathrm{Mg} / \mathrm{Fe}]$. On the other hand, the high $[(\mathrm{Sc}, \mathrm{Ti}) / \mathrm{Fe}]$ ratios indicate nucleosynthesis in a highentropy environment that is likely to be realized in an aspherical explosion.

$\star[\mathbf{T i} / \mathbf{F e}]:$ The disagreement in $[\mathrm{Ti} / \mathrm{Fe}]$ could be solved by taking into account the NLTE effect. The NLTE abundance determination by $\mathrm{Ti} \mathrm{I}$ and $\mathrm{Ti}$ II lines still needs to be improved. However, if we adopt the lines of equal ionization stages as recommended by Bergemann (2011), [Ti II/Fe II] $(=+0.4)$ is consistent with the SN model.

CS 22897-008: The metallicity is $[\mathrm{Fe} / \mathrm{H}]=$ -3.50 and the abundance ratios are shown in Figure 2d (Cavrel et al. 2004; Spite et al. 2005, 2006; Andrievskv et al. 2007, 2008, 2010; Spite et al. 2012; Yong et al. 2013). The abundance pattern except for $[\mathrm{K} / \mathrm{Fe}]$ is well reproduced by an SN model with $E_{51}=20$, $M_{\text {rem }}=4.22 \mathrm{M}_{\odot}$, and $M_{\mathrm{ej}}(\mathrm{Fe})=5.65 \times 10^{-2} \mathrm{M}_{\odot}($ solid line). The abundance pattern and thus the SN model are similar to those of CS 22189-009. A model with lower explosion energy of $E_{51}=5$ (dashed line) also reproduces the abundance pattern, except for the worse agreement of Na. The distinct difference between the models with $E_{51}=20$ and 5 is seen in the abundance of $\mathrm{F}$ because the maximum temperature of the N-rich layer is higher for the higher explosion energy $(\S 4.2)$.

BS 16545-089: The metallicity is $[\mathrm{Fe} / \mathrm{H}]=-3.50$ and the abundance ratios are shown in Figure $2 \mathrm{e}$ (Cohen et al. 2004; Lai et al. 2008; Aoki et al. 2009; Yong et al. 2013). For CNO elements, only the loose upper limits are obtained for $[(\mathrm{C}, \mathrm{N}) / \mathrm{Fe}]$. The $[\mathrm{Ti} / \mathrm{Fe}]$ ratio given in Cohen et al. (2004) and Lai et al. (2008) is different, so its priority is low for our model fitting. The abundance pattern except for $[\mathrm{Co} / \mathrm{Fe}]$ is well reproduced by an $\mathrm{SN}$ model with $E_{51}=20, M_{\mathrm{rem}}=4.02 \mathrm{M}_{\odot}$, and $M_{\text {ej }}(\mathrm{Fe})=1.07 \times 10^{-1} \mathrm{M}_{\odot}$.

$\star[\mathbf{C o} / \mathbf{F e}]:[\mathrm{Co} / \mathrm{Fe}]$ is enhanced by the low-density and $Y_{\mathrm{e}}$ modification but still underabundant. It is known that $[\mathrm{Co} / \mathrm{Fe}]$ has an NLTE effect (Bergemann et al. 2010) but the correction is opposite to the improvement. Although the $[\mathrm{Co} / \mathrm{Fe}]$ can be enhanced as high as $\sim+0.9$ by nu-

\footnotetext{
${ }^{7}$ We note that $\mathrm{F}$ could not be enhanced in a red supergiant with the far-off $\mathrm{N}$-rich layer even if $\mathrm{N}$ is enhanced in the $\mathrm{SN}$ progenitor.

8 According to the swept-up H mass (Thornton et al. 1998, see also Tominaga et al. 2007b) and standard big bang nucleosynthesis (e.g., Coc et al. 2004), the swept-up mass of primordial ${ }^{7} \mathrm{Li}$ is $\sim 5.8 \times 10^{-6} \mathrm{M}_{\odot}$ for an SN explosion with $E_{51}=1$.
} 


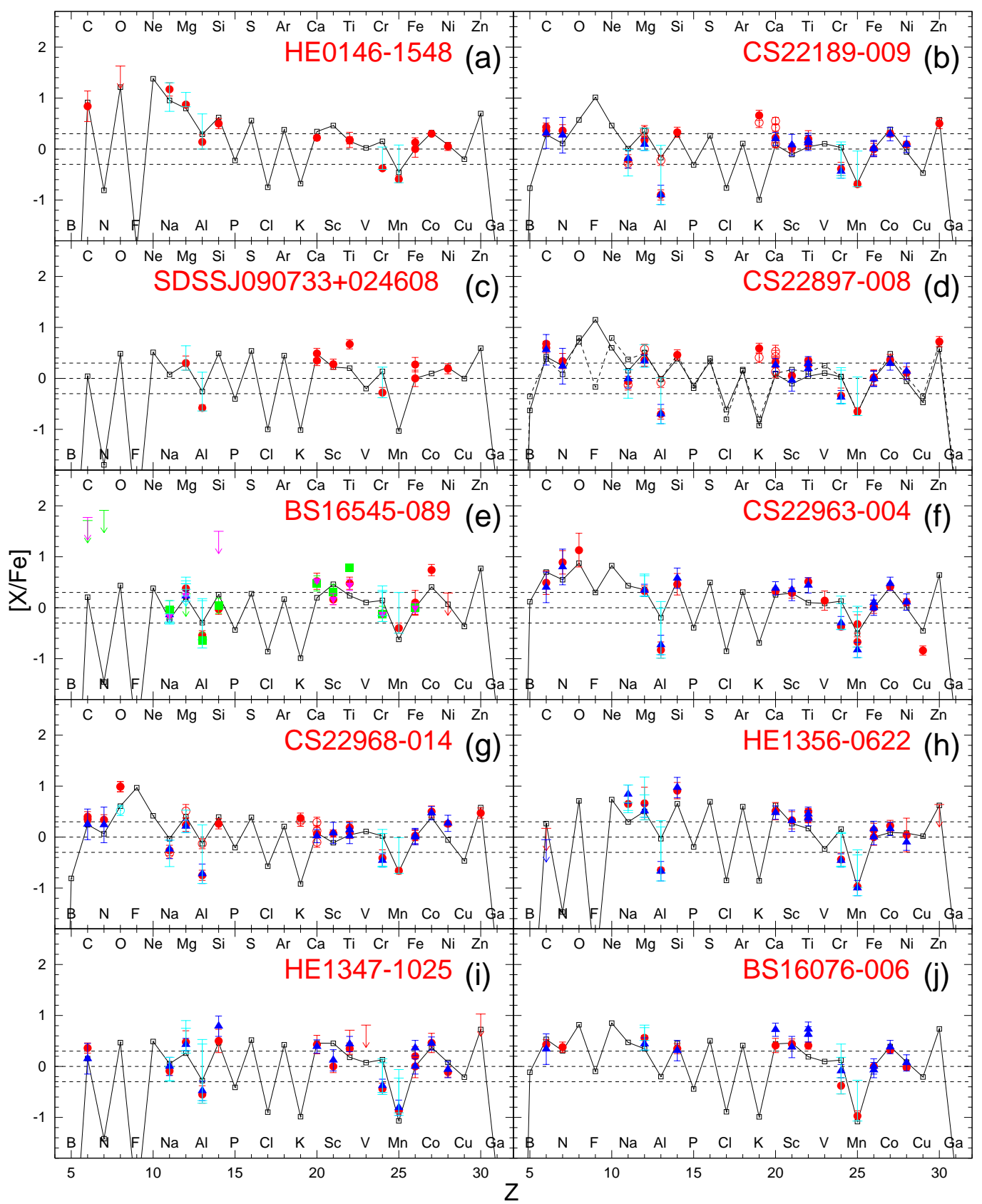

FIG. 2.- Comparison between the abundance patterns of the individual EMP stars (1D-LTE: red/blue/green/magenta/yellow filled points with bars, 3D or NLTE: red/blue/green/magenta/yellow open points with bars, and expediently-corrected [see the text for the detail]: cyan bars or open points with bars) and the Pop III SN models (lines with open squares). The colors of points represent reference papers (see Table 2 in detail). The properties and legends of Pop III SN models are summarized in Table 3

cleosynthesis in a high-entropy and slightly $p$-rich hot bubble or accretion disk (e.g., Pruet et al. 2004, 2005), $[\mathrm{Co} / \mathrm{Fe}]$ in the integrated SN ejecta is enhanced only up to +0.4 (Fröhlich et al. 2006b) a; Izutani \& Umeda 2010). Such high $[\mathrm{Co} / \mathrm{Fe}](\sim+0.9)$ is reproduced if the mixing of the SN ejecta is inhomogeneous and a next-generation star forms from gases enriched only by a portion of the $\mathrm{SN}$ ejecta with high $[\mathrm{Co} / \mathrm{Fe}]$.

CS 22963-004: The metallicity is $[\mathrm{Fe} / \mathrm{H}]=-3.54$ and the abundance ratios are shown in Figure 2] (Lai et al. 2008; Yong et al. 2013). The abundance pattern except for $[(\mathrm{Ti}, \mathrm{Cu}) / \mathrm{Fe}]$ is well reproduced by an $\mathrm{SN}$ model with $E_{51}=5, M_{\mathrm{rem}}=4.75 \mathrm{M}_{\odot}$, and $M_{\mathrm{ej}}(\mathrm{Fe})=$
$2.85 \times 10^{-2} \mathrm{M}_{\odot}$.

$\star[\mathbf{C u} / \mathbf{F e}]:$ Lai et al. (2008) indicated the overproduction of $\mathrm{Cu}$ in the $\mathrm{SN}$ model and proposed that $[\mathrm{Cu} / \mathrm{Fe}]$ in theoretical models can be reduced by appropriate choice of $M_{\text {cut }}$. However, $M_{\text {cut }}$ is a fundamental parameter affecting other elements as well. For example, the choice of $M_{\text {cut }}$ yielding lower $[\mathrm{Cu} / \mathrm{Fe}]$ leads lower $[\mathrm{Zn} / \mathrm{Fe}]$. On the other hand, the 3D effect of the stellar atmosphere model reduces the observed $[\mathrm{Cu} / \mathrm{Fe}]$ for a dwarf star (Bonifacio et al. 2009a). This worsens the agreement. However, the effect for a subgiant star as CS 22963-004 is not clarified and the effect is weaker for lower metallicity. Furthermore, there has been no examination of 


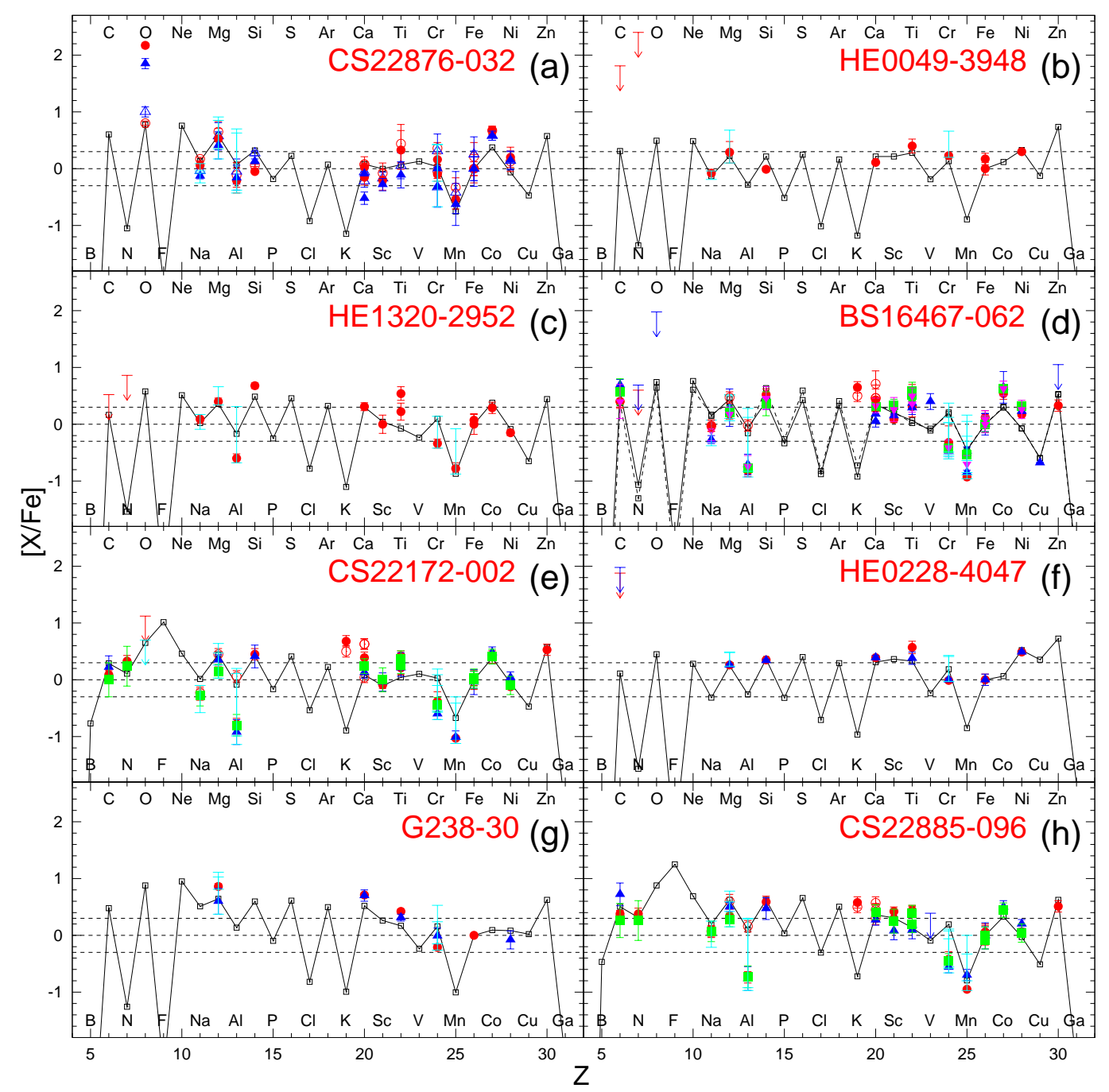

FIG. 3.- Same as Fig. 2

the NLTE effect on $\mathrm{Cu}$. A 3D-NLTE study is needed to minutely investigate on $\mathrm{Cu}$.

$\star \quad[\mathbf{O} / \mathbf{F e}]:[\mathrm{O} / \mathrm{Fe}]$ of $\mathrm{CS} 22963-004$ is estimated with UV OH lines (Lai et al. 2008). The O abundance derived from these lines could be reduced by the 3D effect as much as 0.7 (e.g., Asplund 2005; González Hernández et al. 2010). The SN model is consistent with the observation, even if $[\mathrm{O} / \mathrm{Fe}]$ is reduced by 0.7 dex.

CS 22968-014: The metallicity is $[\mathrm{Fe} / \mathrm{H}]=$ -3.58 and the abundance ratios are shown in Figure 2p (Cavrel et al. 2004; Spite et al. 2005, 2006; Andrievskv et al. 2007, 2008, 2010; Spite et al. 2012; Yong et al. 2013). The abundance pattern except for $[\mathrm{K} / \mathrm{Fe}]$ is well reproduced by an SN model with $E_{51}=20$, $M_{\mathrm{rem}}=3.84 \mathrm{M}_{\odot}$, and $M_{\mathrm{ej}}(\mathrm{Fe})=8.61 \times 10^{-2} \mathrm{M}_{\odot}$. The improvement of $[\mathrm{K} / \mathrm{Fe}]$ could be obtained with including the $p$-rich ejecta produced by neutrino processes (see also a discussion on CS 22189-009). The abundance pattern and thus the SN model are similar to CS 22189-009.

$\star[\mathbf{O} / \mathbf{F e}]$ : Cayrel et al. (2004) presented $[\mathrm{O} / \mathrm{Fe}]$ tentatively corrected by the $3 \mathrm{D}$ effect which is computed by following Nissen et al. (2002). The corrected $[\mathrm{O} / \mathrm{Fe}]$ is in good agreement with the SN model.

HE 1356-0622: The metallicity is $[\mathrm{Fe} / \mathrm{H}]=-3.58$ and the abundance ratios are shown in Figure $2 \mathrm{~h}$ (Cohen et al. 2008; Yong et al. 2013). For CNO elements, only an upper limit to $[\mathrm{C} / \mathrm{Fe}]$ is obtained as +0.2 , which is rather low compared with $[\mathrm{Na} / \mathrm{Fe}] \sim 0.6$. The abundance pattern except for $[\mathrm{Na} / \mathrm{Fe}]$ is well reproduced by an $\mathrm{SN}$ model with $E_{51}=5, M_{\mathrm{rem}}=1.96 \mathrm{M}_{\odot}$, and $M_{\mathrm{ej}}(\mathrm{Fe})=1.11 \times 10^{-1} \mathrm{M}_{\odot}$. The high $[(\mathrm{Sc}, \mathrm{Ti}) / \mathrm{Fe}]$ and the upper limit to $[\mathrm{Zn} / \mathrm{Fe}]$ are reproduced with large $M_{\text {cut }}$.

$\star[\mathrm{Na} / \mathrm{C}]$ : The upper limit of $[\mathrm{C} / \mathrm{Fe}]$ makes it difficult to reproduce high $[\mathrm{Na} / \mathrm{Fe}]$. Such high $[\mathrm{Na} / \mathrm{C}](>+0.4)$ could be reproduced if $\mathrm{C}$ is converted to $\mathrm{N}$ by mixing in HE $1356-0622$ as in the NEMP stars (§3.3). Also, the underproduction of $\mathrm{Na}$ could be improved with a lower explosion energy because weak explosive burning suppresses the destruction of Na. However, such an explosion with $E_{51} \leq 1$ is difficult to reproduce $[(\mathrm{Sc}, \mathrm{Ti}) / \mathrm{Fe}$.

HE 1347-1025: The metallicity is $[\mathrm{Fe} / \mathrm{H}]=-3.62$ and the abundance ratios are shown in Figure 2. (Cohen et al. 2008; Yong et al. 2013). The abundance pattern except for $[\mathrm{Sc} / \mathrm{Fe}]$ is well reproduced by an $\mathrm{SN}$ model with $E_{51}=5, M_{\mathrm{rem}}=4.00 \mathrm{M}_{\odot}$, and $M_{\mathrm{ej}}(\mathrm{Fe})=$ 
$9.85 \times 10^{-2} \mathrm{M}_{\odot}$.

$\star[\mathrm{Sc} / \mathbf{F e}]$ and $[\mathrm{Ti} / \mathbf{F e}]$ : The overproduction of $\mathrm{Sc}$ could be suppressed by a model with slightly lower entropy but such a model would lead to the underproduction of $\mathrm{Ti}$.

BS 16076-006: The metallicity is $[\mathrm{Fe} / \mathrm{H}]=-3.62$ and the abundance ratios are shown in Figure 2] (Bonifacio et al. 2009b; Yong et al. 2013). The star shows $[\mathrm{C} / \mathrm{N}] \sim 0$. Since the star is a cool subgiant (Bonifacio et al. 2007), the observed $\mathrm{N}$ is likely to be synthesized in the SN progenitor due to the enhanced mixing. The abundance pattern is well reproduced by an $\mathrm{SN}$ model with $E_{51}=5, M_{\mathrm{rem}}=3.87 \mathrm{M}_{\odot}$, and $M_{\mathrm{ej}}(\mathrm{Fe})=5.10 \times 10^{-2} \mathrm{M}_{\odot}$.

CS 22876-032AB: The abundance ratios are presented in González Hernández et al. (2008). These two stars with similar masses compose a binary system. The abundance patterns of these stars are consistent within the errors, except for $\mathrm{Li}$, and their metallicities are $[\mathrm{Fe} / \mathrm{H}]=-3.66$ (the red and blue circles in Fig. 3a). The abundance patterns are well reproduced by an SN model with $E_{51}=20, M_{\text {rem }}=4.49 \mathrm{M}_{\odot}$, and $M_{\text {ej }}(\mathrm{Fe})=4.24 \times 10^{-2} \mathrm{M}_{\odot}$.

$\star[\mathbf{O} / \mathbf{F e}]:$ The $3 \mathrm{D}$ effect largely reduces $[\mathrm{O} / \mathrm{Fe}]$ derived from the UV $\mathrm{OH}$ lines. If the $3 \mathrm{D}$ correction would be negligible, the high $1 \mathrm{D}[\mathrm{O} / \mathrm{Fe}]$ indicates an $\mathrm{SN}$ with small $M_{\text {ej }}(\mathrm{Fe})$ that leads to high $[\mathrm{C} / \mathrm{Fe}]$ (see also $\S 3.2$ ).

HE 0049-3948: The metallicity is $[\mathrm{Fe} / \mathrm{H}]=-3.62$ and the abundance ratios are shown in Figure [3b (Yong et al. 2013). For CNO elements, only loose upper limits to $[(\mathrm{C}, \mathrm{N}) / \mathrm{Fe}]$ are obtained. The abundance pattern is well reproduced by an SN model with $E_{51}=20$, $M_{\mathrm{rem}}=4.28 \mathrm{M}_{\odot}$, and $M_{\mathrm{ej}}(\mathrm{Fe})=8.34 \times 10^{-2} \mathrm{M}_{\odot}$. The slightly high $[\mathrm{Ni} / \mathrm{Fe}]$ is reproduced by ejection of material with low $Y_{\mathrm{e}}(<0.5)$.

HE 1320-2952: The metallicity is $[\mathrm{Fe} / \mathrm{H}]=-3.69$ and the abundance ratios are shown in Figure 3 ; (Yong et al. 2013). For CNO elements, marginal upper limits to $[(\mathrm{C}, \mathrm{N}) / \mathrm{Fe}]$ are obtained. The abundance pattern is well reproduced by an SN model with $E_{51}=10$, $M_{\text {rem }}=2.73 \mathrm{M}_{\odot}$, and $M_{\mathrm{ej}}(\mathrm{Fe})=1.30 \times 10^{-1} \mathrm{M}_{\odot}$.

BS 16467-062: The metallicity is $[\mathrm{Fe} / \mathrm{H}]=-3.74$ and the abundance ratios are shown in Figure 31 (Cavrel et al. 2004; Spite et al. 2005, 2006; Andrievskv et al. 2007, 2008; Lai et al. 2008; Cohen et al. 2008; Andrievsky et al. 2010; Spite et al. 2012; Yong et al. 2013). The abundance pattern except for $[(\mathrm{K}, \mathrm{V}, \mathrm{Ni}) / \mathrm{Fe}]$ is well reproduced by an $\mathrm{SN}$ model with $E_{51}=20, M_{\mathrm{rem}}=4.51 \mathrm{M}_{\odot}$, and $M_{\mathrm{ej}}(\mathrm{Fe})=4.36 \times 10^{-2} \mathrm{M}_{\odot}$ (solid line). A model with the lower explosion energy of $E_{51}=5$ also well reproduces the abundance pattern (dashed line). It is difficult to distinguish the SN models with different explosion energies for this star $(\S 4.2)$.

$\star[(\mathbf{V}, \mathbf{N i}) / \mathbf{F e}]$ : The abundance ratios $[(\mathrm{V}, \mathrm{Ni}) / \mathrm{Fe}]$ depend on $Y_{\mathrm{e}}$ and the mass of the complete Si-burning layer. In complete Si burning, since nuclei are photodisintegrated and re-synthesized, the isotopic ratios depend mainly on $Y_{\mathrm{e}}$. The synthesis of $\mathrm{V}$ and $\mathrm{Ni}$ is efficient in the regions where $Y_{\mathrm{e}}$ is close to $Y_{\mathrm{e}}$ of the parent radioisotopes, e.g., ${ }^{51} \mathrm{Mn}$ for $\mathrm{V}$ and ${ }^{58} \mathrm{Cu}$ for $\mathrm{Ni}$, or $Y_{\mathrm{e}}$ of the stable isotopes, e.g., ${ }^{58} \mathrm{Ni}$ for $\mathrm{Ni}$. Since $Y_{\mathrm{e}}$ also affects the abundances of other Fe-peak elements, the abundance ratios among the Fe-peak elements could constrain $Y_{\mathrm{e}}$ distribution in the SN ejecta. A multi-dimensional effect also influences the synthesis of these elements.

CS 22172-002: The metallicity is $[\mathrm{Fe} / \mathrm{H}]=$ -3.74 and the abundance ratios are shown in Figure 3e Cavrel et al. 2004; Spite et al. 2005, 2006; Andrievskv et al. 2007, 2008, 2010; Spite et al. 2012; Yong et al. 2013). The abundance pattern except for $[\mathrm{K} / \mathrm{Fe}]$ is well reproduced by an SN model with $E_{51}=20$, $M_{\text {rem }}=3.87 \mathrm{M}_{\odot}$, and $M_{\mathrm{ej}}(\mathrm{Fe})=7.76 \times 10^{-2} \mathrm{M}_{\odot}$. The abundance pattern and thus the SN model are similar to CS 22189-009.

HE 0228-4047: The metallicity is $[\mathrm{Fe} / \mathrm{H}]=-3.75$ and the abundance ratios are shown in Figure 3: (Yong et al. 2013). The abundance patterns derived with the surface gravity of subgiant and dwarf stars are similar. For CNO elements, only a loose upper limit to $[\mathrm{C} / \mathrm{Fe}]$ is obtained. The abundance patterns are well reproduced by an $\mathrm{SN}$ model with $E_{51}=20, M_{\text {rem }}=3.27 \mathrm{M}_{\odot}$, and $M_{\mathrm{ej}}(\mathrm{Fe})=1.36 \times 10^{-1} \mathrm{M}_{\odot}$. The high $[\mathrm{Ni} / \mathrm{Fe}]$ is reproduced by ejection of low- $Y_{\mathrm{e}}$ material and the high $[\mathrm{Ti} / \mathrm{Fe}]$ indicates nucleosynthesis in a high-entropy environment.

G 238-30: The metallicity is $[\mathrm{Fe} / \mathrm{H}]=-3.77$ and the abundance ratios are shown in Figure $3 \mathrm{~g}$ (Stephenson \& Green 2002; Ishigaki et al. 2010). The abundance ratios $[(\mathrm{C}, \mathrm{N}, \mathrm{O}) / \mathrm{Fe}]$ are not available. The abundance pattern is well reproduced by an SN model with $E_{51}=5, M_{\text {rem }}=2.55 \mathrm{M}_{\odot}$, and $M_{\mathrm{ej}}(\mathrm{Fe})=6.80 \times$ $10^{-2} \mathrm{M}_{\odot}$.

CS 22885-096: The metallicity is $[\mathrm{Fe} / \mathrm{H}]=-3.77$ and the abundance ratios are shown in Figure 3h (Cavrel et al. 2004; Spite et al. 2005, 2006; Andrievskv et al. 2007, 2008; Cohen et al. 2008; Andrievsky et al. 2010; Spite et al. 2012; Yong et al. $2013)$. Since the star shows $[\mathrm{C} / \mathrm{N}] \sim 0$ and the mixing in CS 22885-096 is not strong (Spite et al. 2005, 2006), $\mathrm{N}$ is likely to be synthesized in the SN progenitor due to the enhanced mixing. The abundance pattern except for $[\mathrm{K} / \mathrm{Fe}]$ is well reproduced by an SN model with $E_{51}=20$, $M_{\mathrm{rem}}=3.76 \mathrm{M}_{\odot}$, and $M_{\mathrm{ej}}(\mathrm{Fe})=4.95 \times 10^{-2} \mathrm{M}_{\odot}$. The abundance pattern and thus the SN model are similar to those of CS 22189-009.

\subsection{Carbon-Enhanced Metal-Poor stars}

The CEMP star is one of the largest subclasses of the MP stars. They are further classified by the abundance of $n$-capture elements: CEMP-s stars with an enhancement of $s$-process elements, CEMP-no stars without any enhancement of $n$-capture elements, and so on (see Beers \& Christlieb 2005 for detail).

The CEMP-s star is most common among CEMP stars and is considered to be originated from an MP star constituting a binary system. A mass transfer from an asymptotic giant branch (AGB) binary companion enhances abundances of carbon and $s$-process elements on the surface of the MP star and transforms the MP star to a CEMP-s star (e.g., Busso et al.|1999; Iwamoto et al. 2004; Suda et al. 2004; Lugaro et al. 2012). A signature of binarity is seen for possibly all CEMP-s stars (Lucatello et al. 2005). The lowest $[\mathrm{Fe} / \mathrm{H}]$ of CEMP-s stars is -3.1 (Johnson \& Bolte 2002).

We focus on 12 CEMP-no stars with $-4.1 \lesssim[\mathrm{Fe} / \mathrm{H}] \lesssim$ $-3.5,[\mathrm{C} / \mathrm{Fe}]>+1.0$, and $[\mathrm{C} / \mathrm{N}] \geq-0.5$. In contrast to the origin of CEMP-s stars, the origin of CEMP-no stars 


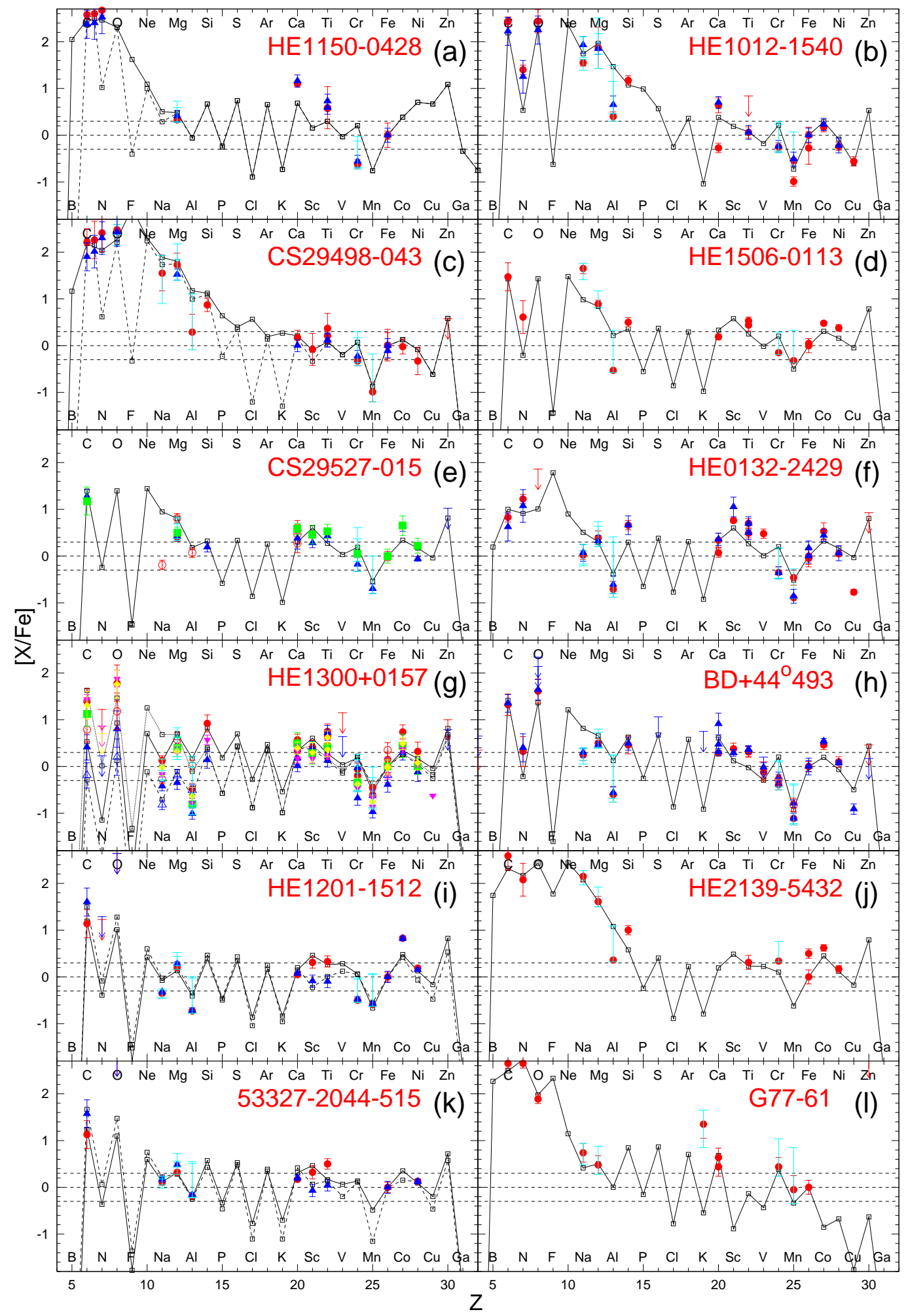

Fig. 4.- Same as Fig. 2 but for the CEMP stars. 
is under debate (Nomoto et al. 2013). The mechanism of carbon enhancement has been proposed as follows:

(1) a faint SN with small ejection of $\mathrm{Fe}$ (Umeda \& Nomoto 2002, 2005; Tominaga et al. 2007b) ${ }^{9}$

(2) a dark SN without Fe ejection (Limongi \& Chieffi 2003),

(3) mass loss from a rotating massive star (Mevnet et al. 2006),

(4) a mass transfer from an AGB binary companion (e.g., Suda et al. 2004), and

(5) self-enrichment in an MP red giant star (Fujimoto et al. 2000; Campbell et al. 2010).

Among the above 5 models, only the faint SN model attributes the origin of the CEMP-no stars to a single Pop III SN. Other models explain the carbon enhancement but require another $\mathrm{SN}(\mathrm{e})$ to produce elements heavier than $\mathrm{CNO}$ and/or Fe-peak elements. In the latter models, Fe-peak elements are suggested to originate from concurrent enrichment by a normal SN or hypernova (Limongi \& Chieffi 2003) and/or the mass accretion from the interstellar medium (Yoshii 1981). The mass accretion from the interstellar medium could also produce the CEMP star only by itself if the interstellar medium is C-rich.

We adopt a faint SN model as the mechanism of carbon enhancement for the following reasons:

(a) A CEMP-no star, which can be explained only by the faint SN mechanism, has been discovered (Ito et al. 2009, 2013).

(b) Faint $\mathrm{SNe}$ are observed in the present day $\left[M\left({ }^{56} \mathrm{Ni}\right) \sim 10^{-3} \mathrm{M}_{\odot}, \quad\right.$ e.g., $\quad$ SN 1999br: Zampieri et al. 2003, SN 2008ha: Moriva et al. 2010.

(c) Concurrent and equivalent enrichment by two $\mathrm{SNe}$ or massive stars may be rare.

(d) The amount of mass loss from a rapidly rotating $Z=0$ star is small (Ekström et al. 2008).

(e) The CEMP-no stars show low $[\mathrm{Ba} / \mathrm{Fe}] .{ }^{10}$

(f) The binary fraction of the CEMP-no stars is absolutely different from that of the CEMP-s stars (Norris et al. 2013).

(g) There exist CEMP-no subgiants and dwarf stars and thus the self-enrichment mechanism is not applicable for all CEMP-no stars.

\footnotetext{
9 The SN with small ejection of Fe is faint because Fe is synthesized as ${ }^{56} \mathrm{Ni}$ that powers a light curve of the SN.

${ }^{10}$ We note that the abundance of Ba could be suppressed if the binary companion is the low-mass AGB star with $M_{\mathrm{MS}}<1.2 \mathrm{M}_{\odot}$ or massive AGB star with $M_{\mathrm{MS}}>3.5 \mathrm{M}_{\odot}$ (Komiva et al. 2007; Suda \& Fuiimoto 2010).
}

HE 1150-0428: The metallicity is $[\mathrm{Fe} / \mathrm{H}]=-3.47$ and the abundance ratios are shown in Figure 4 a (Cohen et al. 2006; Yong et al. 2013).

$\star[\mathbf{C} / \mathbf{N}]$ and $[(\mathbf{B}, \mathbf{F}) / \mathbf{F e}]$ : Since $\mathrm{N}$ is as enhanced as $[\mathrm{C} / \mathrm{N}] \sim 0$, origin of $\mathrm{N}$ needs to be discussed. If $\mathrm{N}$ is assumed to be synthesized in the SN progenitor by the enhanced mixing of $\mathrm{H}$ into the He layer and the subsequent $\mathrm{CNO}$ cycle, the abundance ratios $[(\mathrm{C}, \mathrm{N}, \mathrm{Mg}, \mathrm{Ti}) / \mathrm{Fe}]$ are well reproduced by an $\mathrm{SN}$ model with $E_{51}=5$, $M_{\mathrm{rem}}=5.83 \mathrm{M}_{\odot}$, and $M_{\mathrm{ej}}(\mathrm{Fe})=3.43 \times 10^{-4} \mathrm{M}_{\odot}($ solid line). However, HE $1150-0428$ shows low ${ }^{12} \mathrm{C} /{ }^{13} \mathrm{C}(\sim 4$, Cohen et al. 2006) in spite of the relatively high surface gravity $[\log (g)=2.54]$. This indicates that a material in the envelope experiences the $\mathrm{CNO}$ cycle, and thus suggests that $\mathrm{N}$ is produced in HE $1150-0428$ as in the NEMP stars $(\S[3.3)$. If $\mathrm{N}$ is assumed to be converted from $\mathrm{C}$ by the CNO cycle in HE 1150-0428, the abundance ratios are well reproduced by an SN model of Model A with $E_{51}=5, M_{\mathrm{rem}}=5.83 \mathrm{M}_{\odot}$, and $M_{\mathrm{ej}}(\mathrm{Fe})=3.51 \times 10^{-4} \mathrm{M}_{\odot}$ (dashed line). The origin of $\mathrm{N}$ might be constrained by $[(\mathrm{B}, \mathrm{F}) / \mathrm{Fe}]$ because $\mathrm{B}$ and $\mathrm{F}$ are synthesized by the hot $\mathrm{CNO}$ cycle and ${ }^{3} \mathrm{He}$ burning if the He/N-rich layer experiences high temperature during the shock propagation.

$\star[\mathbf{C} / \mathbf{M g}]$ : The high $[\mathrm{C} / \mathrm{Mg}]$ is reproduced by mixingand-fallback during the explosion: mixing up to the top of the $\mathrm{O}+\mathrm{Mg}$ layer, i.e., large $M_{\mathrm{mix}}$, and the small ejection from the mixing region, i.e., small $f_{\text {ej. }}$. These result in large $M_{\text {rem }}$.

$\star[\mathbf{C a} / \mathbf{F e}]$ : There remains a discrepancy on $[\mathrm{Ca} / \mathrm{Fe}]$. The large $M_{\text {cut }}$ could enhance $[\mathrm{Ca} / \mathrm{Fe}]$ but lead to too high $[\mathrm{Mg} / \mathrm{Fe}]$ and too low $[\mathrm{Ti} / \mathrm{Fe}]$.

HE 1012-1540: The metallicity is $[\mathrm{Fe} / \mathrm{H}]=-3.48$ and the abundance ratios are shown in Figure $4 \mathrm{~b}$ (Cohen et al. 2008; Yong et al. 2013). The abundance pattern except for $[\mathrm{N} / \mathrm{Fe}]$ is well reproduced by an $\mathrm{SN}$ model with $E_{51}=20, M_{\mathrm{rem}}=4.81 \mathrm{M}_{\odot}$, and $M_{\mathrm{ej}}(\mathrm{Fe})=$ $1.11 \times 10^{-3} \mathrm{M}_{\odot}$.

$\star[(\mathbf{C}, \mathbf{M g}) / \mathbf{F e}]:$ The high $[(\mathrm{C}, \mathrm{Mg}) / \mathrm{Fe}]$ is reproduced by the mixing-and-fallback model that assumes mixing up to the middle of the $\mathrm{O}+\mathrm{Mg}$ layer and small ejection from the mixing region.

$\star[\mathbf{C} / \mathbf{N}]$ : The $\mathrm{N}$ abundance is as low as $[\mathrm{N} /(\mathrm{C}, \mathrm{O})] \lesssim$ -1.0 and thus the faint $\mathrm{SN}$ mechanism is most preferable among the $\mathrm{C}$ enhancement mechanisms. The underproduction of $\mathrm{N}$ is improved if $15 \%$ of $\mathrm{C}$ in the He layer is converted to $\mathrm{N}$ due to slightly efficient mixing at the boundary of $\mathrm{H}$ and He layers in the SN progenitor.

CS 29498-043: The metallicity is $[\mathrm{Fe} / \mathrm{H}]=-3.52$ and the abundance ratios are shown in Figure $4 \mathrm{c}$ (Aoki et al.|2004; Y Yong et al. 2013). The abundance pattern is well reproduced by the SN model A with $E_{51}=20$, $M_{\text {rem }}=5.11 \mathrm{M}_{\odot}$, and $M_{\mathrm{ej}}(\mathrm{Fe})=9.08 \times 10^{-4} \mathrm{M}_{\odot}($ solid line).

$\star[\mathbf{C} / \mathbf{N}]:$ The $\mathrm{N}$ abundance is as high as $[\mathrm{N} / \mathrm{C}] \sim 0$, but the origin of $\mathrm{N}$ cannot be constrained, since there is no observation of $\mathrm{Li}$ or ${ }^{13} \mathrm{C}$. The abundance pattern is also well reproduced with the SN model B if that the mixing in CS 29498-043 is assumed to enhance [N/Fe] as in HE 1150-0428 (dashed line); however, it needs to be confirmed whether $[\mathrm{C} / \mathrm{N}] \sim 0$ is realized. The differences between Models $\mathrm{A}$ and $\mathrm{B}$ appear in $[(\mathrm{B}, \mathrm{F}) / \mathrm{Fe}]$.

HE 1506-0113: The metallicity is $[\mathrm{Fe} / \mathrm{H}]=-3.54$ and the abundance ratios are shown in Figure $4 \mathrm{~d}$ 
(Yong et al. 2013). The abundance pattern except for $[(\mathrm{N}, \mathrm{Na}, \mathrm{Ni}) / \mathrm{Fe}]$ is well reproduced by an $\mathrm{SN}$ model with $E_{51}=20, M_{\mathrm{rem}}=5.15 \mathrm{M}_{\odot}$, and $M_{\mathrm{ej}}(\mathrm{Fe})=6.80 \times$ $10^{-3} \mathrm{M}_{\odot}$. Although the NLTE abundance determination reduces $[\mathrm{Na} / \mathrm{Fe}]$, the underproduction of $\mathrm{Na}$ is not solved. The agreement of $[\mathrm{Ni} / \mathrm{Fe}]$ is improved if low $-Y_{\mathrm{e}}$ material is ejected.

$\star[\mathbf{C} / \mathbf{N}]:$ The $\mathrm{N}$ abundance is as low as $[\mathrm{N} / \mathrm{C}] \lesssim-0.9$ and thus the faint $\mathrm{SN}$ mechanism is favored among the $\mathrm{C}$ enhancement mechanisms. [N/Fe] could be reproduced if $15 \%$ of $\mathrm{C}$ in the He layer is converted to $\mathrm{N}$ due to slightly efficient mixing at the boundary of $\mathrm{H}$ and $\mathrm{He}$ layers in the SN progenitor.

CS 29527-015: The metallicity is $[\mathrm{Fe} / \mathrm{H}]=-3.55$ and the abundance ratios are shown in Figure 4 (Andrievskv et al. 2007, 2008; Bonifacio et al. 2009b; Andrievsky et al. 2010; Spite et al. 2012; Yong et al. 2013). The abundance pattern except for $[\mathrm{Na} / \mathrm{Fe}]$ is well reproduced by an $\mathrm{SN}$ model with $E_{51}=20, M_{\text {rem }}=$ $5.14 \mathrm{M}_{\odot}$, and $M_{\mathrm{ej}}(\mathrm{Fe})=6.52 \times 10^{-3} \mathrm{M}_{\odot}$.

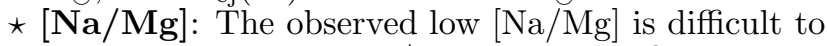
be reproduced unless the $\mathrm{Na} / \mathrm{Mg}$ ratio in the $\mathrm{O}+\mathrm{Mg}$ layer of the progenitor star is reduced by, e.g., quasi-static $\mathrm{C}$ burning at higher temperature. Thus, the agreement is slightly improved with the $40 \mathrm{M}_{\odot}$ model $(\S 4.1)$.

HE 0132-2429: The metallicity is $[\mathrm{Fe} / \mathrm{H}]=-3.70$ and the abundance ratios are shown in Figure 4f (Cohen et al. 2008; Yong et al. 2013). The abundance pattern except for $[(\mathrm{Na}, \mathrm{V}, \mathrm{Cu}) / \mathrm{Fe}]$ is well reproduced by an $\mathrm{SN}$ model with $E_{51}=20, M_{\mathrm{rem}}=5.39 \mathrm{M}_{\odot}$, and $M_{\text {ej }}(\mathrm{Fe})=1.24 \times 10^{-2} \mathrm{M}_{\odot}$.

$\star[\mathbf{C} / \mathbf{N}]$ : The observed $[\mathrm{N} / \mathrm{C}]$ is as high as $\sim 0.2$. In the above model, we assume that $\mathrm{N}$ is synthesized by the enhanced mixing in the supernova progenitor, since there is no constraint on the origin of enhanced $\mathrm{N}$ and it is non-trivial whether $[\mathrm{C} / \mathrm{N}] \sim 0$ is realized by the mixing in HE 0132-2429.

* [Cu/Fe]: As mentioned for CS 22963-004, small $M_{\text {cut }}$ can yield low $[\mathrm{Cu} / \mathrm{Fe}]$ but would lead to the reduction of $[(\mathrm{Sc}, \mathrm{Ti}, \mathrm{V}) / \mathrm{Fe}]$.

$\star[\mathrm{Na} / \mathrm{Fe}]:$ The disagreement of $[\mathrm{Na} / \mathrm{Fe}]$ could be improved if the convective shell has higher temperature during quasi-static $\mathrm{C}$ burning.

HE 1300+0157: The metallicity is $[\mathrm{Fe} / \mathrm{H}]=-3.76$ and the abundance ratios are shown in Figure 4r (Barklem et al. 2005; Frebel et al. 2007; Beers et al. 2007; Cohen et al. 2008; Yong et al. 2013). Among the many papers working on $\mathrm{HE} 1300+0157$, we construct an SN model to reproduce the abundance ratios in Frebel et al. (2007) because they intensively studied HE $1300+0157$.

The abundance pattern for surface gravity of a subgiant star is well reproduced by the SN model A (solid line) with $E_{51}=20, M_{\text {rem }}=3.25 \mathrm{M}_{\odot}$, and $M_{\mathrm{ej}}(\mathrm{Fe})=$ $5.21 \times 10^{-2} \mathrm{M}_{\odot}$, except for $[(\mathrm{Na}, \mathrm{Cu}) / \mathrm{Fe}]$. On the other hand, the abundance pattern for surface gravity of a dwarf star is reproduced by an SN model without mixingand-fallback. The properties of the SN model B (dashed line) are $E_{51}=20, M_{\mathrm{rem}}=2.16 \mathrm{M}_{\odot}$, and $M_{\mathrm{ej}}(\mathrm{Fe})=$ $3.42 \times 10^{-1} \mathrm{M}_{\odot}$. We also present an $\mathrm{SN}$ model $\mathrm{C}$ for the abundance patterns without $3 \mathrm{D}$ effects on $[(\mathrm{C}, \mathrm{O}) / \mathrm{Fe}]$. The properties are $E_{51}=20, M_{\text {rem }}=5.57 \mathrm{M}_{\odot}$, and $M_{\mathrm{ej}}(\mathrm{Fe})=3.58 \times 10^{-3} \mathrm{M}_{\odot}($ dotted line $)$.
$\star[\mathbf{C} / \mathbf{F e}]$ : Since $[\mathrm{C} / \mathrm{Fe}]$ for the dwarf surface gravity (model B) is lower than that for the subgiant surface gravity (model A), the resultant $M_{\mathrm{ej}}(\mathrm{Fe})$ for model $\mathrm{B}$ is $\sim 7$ times larger than for model $\mathrm{A}$. The discrimination between models $\mathrm{A}$ and $\mathrm{B}$ is important to constrain $M_{\mathrm{ej}}(\mathrm{Fe})$.

$\mathrm{BD}+\mathbf{4 4}^{\circ}$ 493: The metallicity is $[\mathrm{Fe} / \mathrm{H}]=-3.78$ and the abundance ratios are shown in Figure $4 \mathrm{~h}$ (Ito et al. 2009, 2013). ${ }^{11}$ The abundance pattern except for $[(\mathrm{N}, \mathrm{Na}, \mathrm{Ti}, \mathrm{Cu}) / \mathrm{Fe}]$ is well reproduced by an SN model with $E_{51}=5, M_{\mathrm{rem}}=5.33 \mathrm{M}_{\odot}$, and $M_{\mathrm{ej}}(\mathrm{Fe})=$ $6.17 \times 10^{-3} \mathrm{M}_{\odot}$. The discrepancies on $[(\mathrm{Na}, \mathrm{Ti}, \mathrm{Cu}) / \mathrm{Fe}]$ are improved with the $40 \mathrm{M}_{\odot}$ model $(\S 4.1)$.

$\star[(\mathbf{C}, \mathbf{N}) / \mathbf{F e}]:$ The origin of the $\mathrm{C}$ enhancement in $\mathrm{BD}+44^{\circ} 493$ is clearly constrained to be a faint $\mathrm{SN}$ by virtue of high $[\mathrm{C} / \mathrm{N}](>0)$, low $[\mathrm{C} / \mathrm{O}](<0)$, and low $\mathrm{Ba}$ and $\mathrm{Pb}$ abundance $([\mathrm{Ba} / \mathrm{Fe}]=-0.6$ and $[\mathrm{Pb} / \mathrm{Fe}]<+1.98$, Ito et al. 2013). [N/Fe] could be reproduced if $10 \%$ of $\mathrm{C}$ in the He layer is converted to $\mathrm{N}$ due to slightly enhanced mixing in the SN progenitor.

HE 1201-1512: The metallicity is $[\mathrm{Fe} / \mathrm{H}]=-3.89$ and the abundance ratios are shown in Figure 4. (Yong et al. 2013). Yong et al. (2013) provides two kinds of abundance patterns with surface gravity of dwarf and subgiant stars. The abundance pattern for the dwarf surface gravity is well reproduced by an SN model with $E_{51}=5, M_{\mathrm{rem}}=5.65 \mathrm{M}_{\odot}$, and $M_{\mathrm{ej}}(\mathrm{Fe})=9.17 \times$ $10^{-3} \mathrm{M}_{\odot}$, except for $[\mathrm{Co} / \mathrm{Fe}]$ (solid line). The abundance pattern for the subgiant surface gravity is reproduced by an SN model with $E_{51}=5, M_{\text {rem }}=5.72 \mathrm{M}_{\odot}$, and $M_{\mathrm{ej}}(\mathrm{Fe})=4.53 \times 10^{-3} \mathrm{M}_{\odot}$ (dashed line). The high $[\mathrm{Co} / \mathrm{Fe}]$ is difficult to be reproduced without an inhomogeneous mixing in the SN ejecta (see also a discussion on BS 16545-089 in $\oint 3.1$.

HE 2139-5432: The metallicity is $[\mathrm{Fe} / \mathrm{H}]=-4.02$ and the abundance ratios are shown in Figure 4] (Yong et al. 2013). The abundance pattern except for $[\mathrm{Si} / \mathrm{Fe}]$ is well reproduced by an $\mathrm{SN}$ model with $E_{51}=5$, $M_{\text {rem }}=5.03 \mathrm{M}_{\odot}$, and $M_{\mathrm{ej}}(\mathrm{Fe})=6.78 \times 10^{-4} \mathrm{M}_{\odot}$.

$\star[\mathbf{C} / \mathbf{N}]$ : Although there is no constraint on the origin of $\mathrm{N}$ enhancement, we assume that $\mathrm{N}$ is synthesized by the enhanced mixing in the progenitor because of $[\mathrm{C} / \mathrm{N}] \sim 0$ as in HE 0132-2429.

* [Si/Fe]: $[\mathrm{Si} / \mathrm{Fe}]$ could be enhanced by a large $M_{\text {cut }}$ but such a large $M_{\text {cut }}$ would reduce [Ti/Fe].

53327-2044-515: The metallicity is $[\mathrm{Fe} / \mathrm{H}]=-4.04$ and the abundance ratios are shown in Figure $4 \mathrm{k}$ (Yong et al. 2013; Norris et al. 2013). The abundance pattern for the dwarf surface gravity is well reproduced by an $\mathrm{SN}$ model with $E_{51}=5, M_{\text {rem }}=5.56 \mathrm{M}_{\odot}$, and $M_{\mathrm{ej}}(\mathrm{Fe})=8.51 \times 10^{-3} \mathrm{M}_{\odot}$, except for $[\mathrm{Ti} / \mathrm{Fe}]$ (solid line). Assuming 53327-2044-515 is a subgiant star, the abundance pattern including $[\mathrm{Ti} / \mathrm{Fe}]$ is well reproduced by an $\mathrm{SN}$ model with $E_{51}=5, M_{\text {rem }}=5.66 \mathrm{M}_{\odot}$, and $M_{\text {ej }}(\mathrm{Fe})=3.26 \times 10^{-3} \mathrm{M}_{\odot}($ dashed line $)$.

G 77-61: The metallicity is $[\mathrm{Fe} / \mathrm{H}]=-4.08$ and the abundance ratios are shown in Figure 4. Plez \& Cohen 2005; Beers et al. 2007). Since its surface gravity is $\log (g)=5.05, \mathrm{~N}$ is likely to be synthesized by the enhanced mixing in the progenitor. The abundance pat-

\footnotetext{
11 The progenitor models adopted in the present paper are different from Ito et al. (2013) in order to investigate the effect of the enhanced mixing.
} 


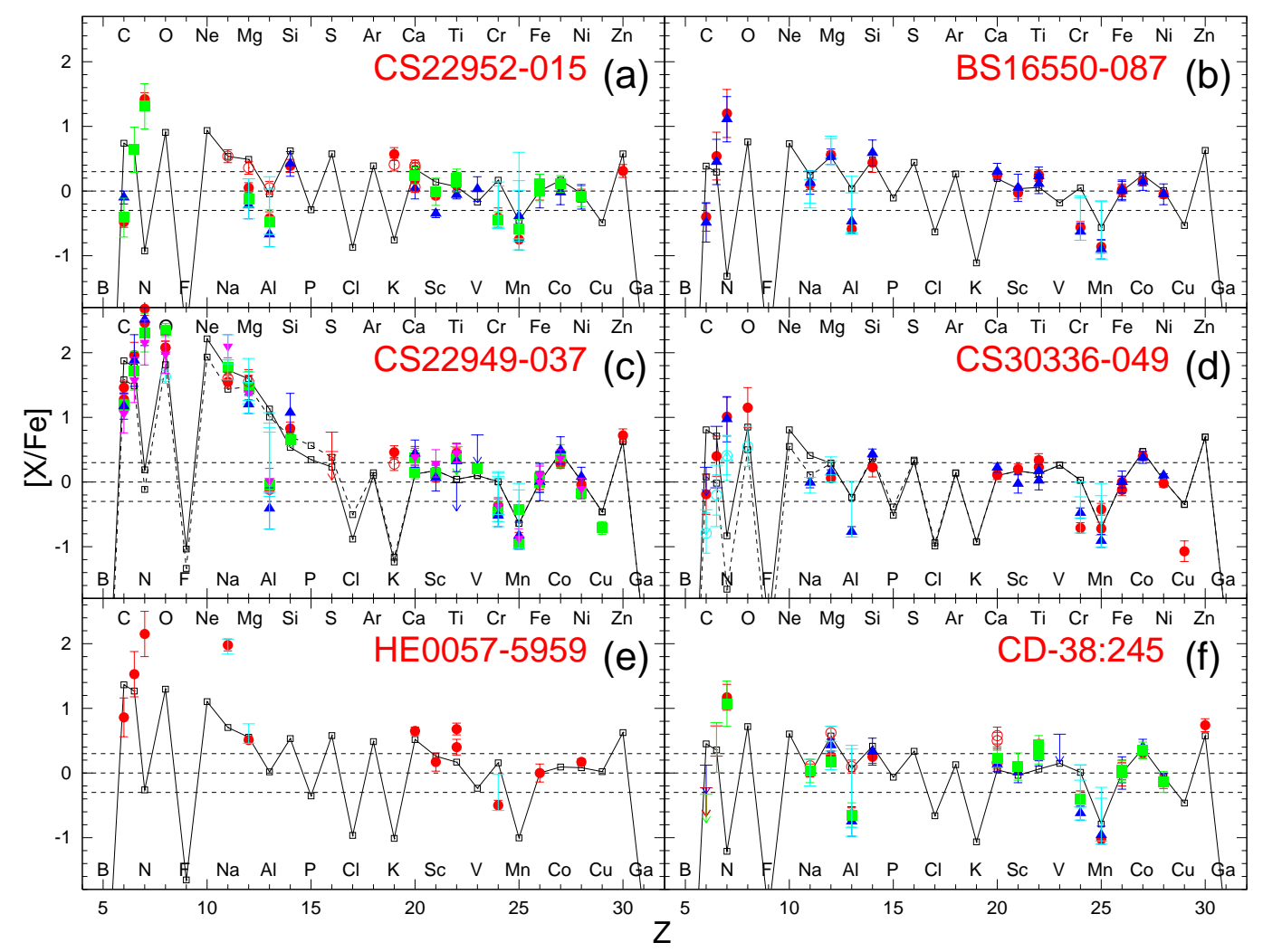

FIG. 5. - Same as Fig. 2 but for the NEMP stars.

tern except for $[\mathrm{K} / \mathrm{Fe}]$ is well reproduced by an SN model with $E_{51}=1, M_{\mathrm{rem}}=6.17 \mathrm{M}_{\odot}$, and $M_{\mathrm{ej}}(\mathrm{Fe})=1.85 \times$ $10^{-4} \mathrm{M}_{\odot}$. The high $[(\mathrm{C}, \mathrm{N}, \mathrm{O}) / \mathrm{Mg}]$ and $[(\mathrm{Ca}, \mathrm{Cr}) / \mathrm{Fe}]$ are reproduced by a large $M_{\text {mix }}$ and small $f_{\mathrm{ej}}$, and a large $M_{\text {cut }}$, respectively.

$\star[\mathbf{K} / \mathbf{F e}]:[\mathrm{K} / \mathrm{Fe}]$ of $\mathrm{G} 77-61$ is remarkably high. Explosive nucleosynthesis in the $p$-rich ejecta might explain such high $K$ abundance, but could also yield a large amount of odd elements (Iwamoto et al. 2006). According to Iwamoto et al. (2006), overabundance of $\mathrm{P}, \mathrm{Cl}, \mathrm{Sc}, \mathrm{V}([\mathrm{X} / \mathrm{Fe}]>+0.5)$ is predicted.

* [(Sc, Ti, Co, Zn)/Fe]: The lack of observed $[(\mathrm{Sc}, \mathrm{Ti}, \mathrm{Co}, \mathrm{Zn}) / \mathrm{Fe}]$ makes it difficult to constrain the explosion energy and entropy during explosive burning.

\subsection{Nitrogen-Enhanced Metal-Poor stars}

Among the EMP stars, there are stars that show enhancement of $\mathrm{N}$, called NEMP stars, which are defined by $[\mathrm{N} / \mathrm{Fe}]>0.5$ and $[\mathrm{C} / \mathrm{N}]<-0.5$ (e.g., Johnson et al. 2007). Half of the NEMP stars at $[\mathrm{Fe} / \mathrm{H}] \lesssim-3.0$ display a signature of mixing in the $\mathrm{Li}$ abundance and the ${ }^{13} \mathrm{C} /{ }^{12} \mathrm{C}$ ratio (Spite et al. 2005, 2006), and the others do not have these measurement due to observational difficulty.

The stars that undergo extra mixing on the red giant branch (RGB) in addition to the first dredge-up are called "mixed" stars. The mixing in the MP stars enhances the conversion from $\mathrm{C}$ to $\mathrm{N}$ via the $\mathrm{CNO}$ cycle. This could be a dominant process to create the NEMP stars at low metallicity. Thus, although the Li abundance or ${ }^{13} \mathrm{C} /{ }^{12} \mathrm{C}$ ratio are not available for some of the NEMP stars, we assume the NEMP stars at $[\mathrm{Fe} / \mathrm{H}] \lesssim-3.5$ are "mixed" stars and construct an SN model that reproduces $[(\mathrm{C}+\mathrm{N}) / \mathrm{Fe}]$ instead of individual $[\mathrm{C} / \mathrm{Fe}]$ and $[\mathrm{N} / \mathrm{Fe}]$.

If the mixing on the RGB is inefficient in the NEMP stars, $\mathrm{N}$ should be enhanced by other mechanisms. The mixing in the AGB stars could enhance [N/C] up to $\sim+2$ (Nishimura et al. 2009; Karakas 2010) and the mass accretion from the AGB binary companion could transform an MP star to an NEMP star (e.g., Pols et al. 2012). However, binarity has been found only for BS 16550-087 (Lai et al. 2008).

On the other hand, mixing in a massive $\mathrm{SN}$ progenitor can enhance $\mathrm{N}$ only up to $[\mathrm{N} / \mathrm{C}] \sim+0.5$ (Iwamoto et al. 2005; Mevnet et al. 2006). Thus the process is not appropriate, by definition, for the origin of the NEMP stars.

CS 22952-015: The metallicity is $[\mathrm{Fe} / \mathrm{H}]=-3.44$ and the abundance ratios are shown in Figure [5] (Cayrel et al.|2004; Honda et al. |2004; |Spite et al.|2005, 2006; Andrievsky et al. 2007, 2008, 2010; Spite et al. 2012; Yong et al. 2013). CS 22952-015 is a mixed star (Spite et al. 2005, 2006) and thus N is likely to be converted from $\mathrm{C}$ in $\mathrm{CS} 22952-015$. The abundance pattern except for $[\mathrm{K} / \mathrm{Fe}]$ is well reproduced by an $\mathrm{SN}$ model with $E_{51}=5, M_{\text {rem }}=4.46 \mathrm{M}_{\odot}$, and $M_{\mathrm{ej}}(\mathrm{Fe})=$ $3.09 \times 10^{-2} \mathrm{M}_{\odot}$. The model demonstrates that $[\mathrm{C} / \mathrm{Fe}]$ of CS 22952-015 before the conversion from $\mathrm{C}$ to $\mathrm{N}$ is less than +1.0 and that CS 22952-015 was the EMP star at birth.

BS 16550-087: The metallicity is $[\mathrm{Fe} / \mathrm{H}]=-3.54$. The abundance ratios are shown in Figure $5 \mathrm{~b}$ (Lai et al. 2008; Yong et al. 2013) but there is no observation of $\mathrm{Li}$ or ${ }^{13} \mathrm{C}$. The abundance pattern is well reproduced 


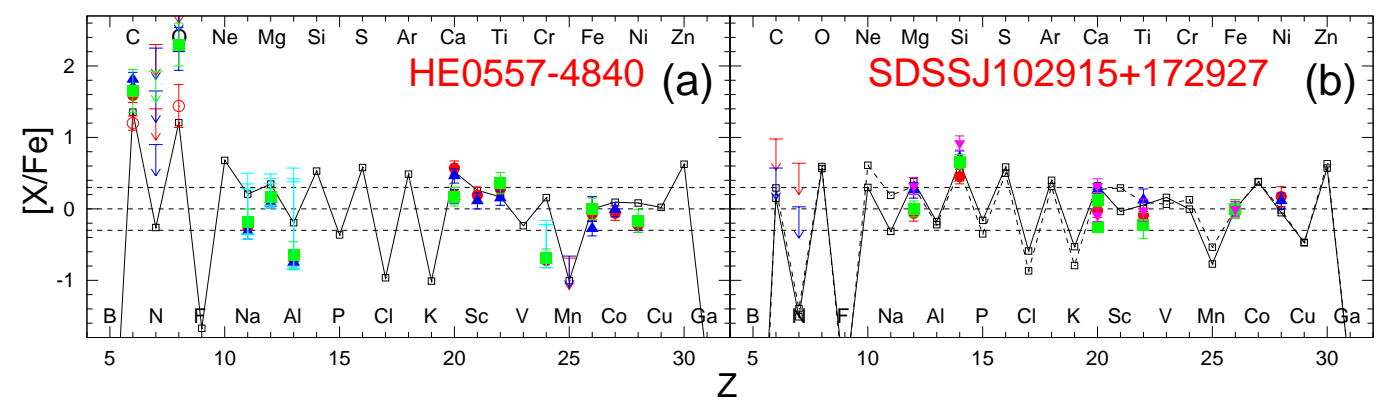

FIG. 6. - Same as Fig. 2 but for the UMP stars.

by an SN model with $E_{51}=10, M_{\text {rem }}=3.14 \mathrm{M}_{\odot}$, and $M_{\mathrm{ej}}(\mathrm{Fe})=7.68 \times 10^{-2} \mathrm{M}_{\odot}$. According to the SN model, BS 16550-087 was the EMP star at birth.

CS 22949-037: The metallicity is $[\mathrm{Fe} / \mathrm{H}]=$ -3.89 and the abundance ratios are shown in Figure 5. (Norris et al. 2001, 2002; Cavrel et al. 2004; Spite et al. 2005, 2006; Andrievskv et al. 2007; Cohen et al. 2008; Andrievsky et al. 2010; Spite et al. 2011, 2012; Yong et al. 2013). CS 22949-037 is a mixed star (Spite et al. 2005, 2006). The abundance pattern except for $[\mathrm{K} / \mathrm{Fe}]$ is well reproduced by an $\mathrm{SN}$ model with $E_{51}=5, M_{\mathrm{rem}}=4.34 \mathrm{M}_{\odot}$, and $M_{\mathrm{ej}}(\mathrm{Fe})=2.42 \times 10^{-3} \mathrm{M}_{\odot}$ (solid line). As mentioned for CS 22189-009 in $\S$ 3.1, the underproduction of K could be solved by including $p$-rich ejecta due to neutrino processes (e.g., Iwamoto et al. 2006; Heger \& Wooslev 2010). The model demonstrates that CS 22949-037 was the CEMP star at birth.

$\star[\mathbf{O} / \mathbf{F e}]$ : Cavrel et al. (2004) also showed $[\mathrm{O} / \mathrm{Fe}]$ tentatively corrected by the $3 \mathrm{D}$ effect. The corrected $[\mathrm{O} / \mathrm{Fe}]$ is reproduced by an SN model with $E_{51}=10$, $M_{\mathrm{rem}}=4.11 \mathrm{M}_{\odot}$, and $M_{\mathrm{ej}}(\mathrm{Fe})=4.85 \times 10^{-3} \mathrm{M}_{\odot}($ dashed line). The SN model has two times larger $M_{\mathrm{ej}}(\mathrm{Fe})$ than the SN model for the uncorrected $[\mathrm{O} / \mathrm{Fe}]$.

Pols et al. (2012) suggests that CS 22949-037 is originated by a mass transfer from an N-rich AGB companion because of insufficient time to alter the surface abundance by extra mixing (Stancliffe 2009). However, there is no indication of the binarity of CS 22949-037 (Depagne et al. 2002). Furthermore, it is not clearly shown whether the abundance pattern of CS 22949-037, e.g., high $[(\mathrm{Na}, \mathrm{Al}) / \mathrm{Mg}]$, is reproduced by this mechanism.

CS 30336-049: The metallicity is $[\mathrm{Fe} / \mathrm{H}]=-4.07$. The abundance ratios are shown in Figure 5 $\mathrm{d}$ (Lai et al. 2008; Yong et al. 2013). The abundance pattern except for $[(\mathrm{Na}, \mathrm{Cu}) / \mathrm{Fe}]$ is well reproduced by an SN model with $E_{51}=5, M_{\mathrm{rem}}=5.01 \mathrm{M}_{\odot}$, and $M_{\mathrm{ej}}(\mathrm{Fe})=2.54 \times 10^{-2} \mathrm{M}_{\odot}$ (solid line).

$\star[\mathbf{C} / \mathbf{N}]$ : The low $[\mathrm{C} / \mathrm{N}](\sim-1.0)$ cannot be realized in the mixing in the massive $\mathrm{SN}$ progenitor, while there is no observation of $\mathrm{Li}$ or ${ }^{13} \mathrm{C}$.

$\star[(\mathbf{C}, \mathbf{N}, \mathbf{O}) / \mathbf{F e}]$ and $[\mathbf{N a} / \mathbf{F e}]:$ The CNO abundance of CS 30336-049 is estimated with $\mathrm{CH}, \mathrm{NH}, \mathrm{OH}$ lines suffered from the large 3D effects (e.g., Asplund 2005). The overproduction of $\mathrm{Na}$ could be resolved if $[(\mathrm{C}, \mathrm{N}, \mathrm{O}) / \mathrm{Fe}]$ is reduced by 0.6 dex due to the $3 \mathrm{D}$ effects (dashed line).

HE 0057-5959: The metallicity is $[\mathrm{Fe} / \mathrm{H}]=$ -4.08 . The abundance ratios are shown in Figure 5 e (Norris et al. 2013; Yong et al. 2013) but there is no observation of ${ }^{13} \mathrm{C}$. The abundance pattern except for $[\mathrm{Na} / \mathrm{Fe}]$ is well reproduced by an SN model with $E_{51}=5$, $M_{\text {rem }}=5.38 \mathrm{M}_{\odot}$, and $M_{\text {ej }}(\mathrm{Fe})=6.80 \times 10^{-3} \mathrm{M}_{\odot}$.

$\star[\mathrm{Na} / \mathrm{Mg}]$ : The high $[\mathrm{Na} / \mathrm{Mg}]$ requires similar number fractions of $\mathrm{Na}$ and $\mathrm{Mg}$ at the $\mathrm{O}+\mathrm{Mg}$ layer in the progenitor. The disagreement of $[\mathrm{Na} / \mathrm{Mg}]$, the surface gravity of $\log (g)=2.65$, and the high Li abundance $(\log \epsilon(\mathrm{Li})=2.12$, Norris et al. 2013) might imply that HE 0057-5959 is originated from the mass transfer from an N-rich AGB companion. However, the binarity is not well constrained and the star shows low $[\mathrm{Ba} / \mathrm{Fe}]$ $(=-0.46$, Yong et al. 2013).

CD-38:245: The metallicity is $[\mathrm{Fe} / \mathrm{H}]=-4.11$ and the abundance ratios are shown in Figure 5: (Norris et al. 2001; Cavrel et al. |2004; Spite et al. 2006; Andrievskv et al. 2007, 2008, 2010; Spite et al. 2012; Yong et al. 2013). CD-38:245 is a mixed star (Spite et al. 2005, 2006). Since CD-38:245 has only an upper limit to $[\mathrm{C} / \mathrm{Fe}]$, a range of $[(\mathrm{C}+\mathrm{N}) / \mathrm{Fe}]$ is estimated by assuming the range of $[\mathrm{C} / \mathrm{Fe}]$ from $-\infty$ to -0.28 . The abundance pattern is well reproduced by an SN model with $E_{51}=20, M_{\mathrm{rem}}=4.07 \mathrm{M}_{\odot}$, and $M_{\text {ej }}(\mathrm{Fe})=6.10 \times 10^{-2} \mathrm{M}_{\odot}$.

\subsection{Ultra Metal-Poor stars}

The UMP stars are defined by the metallicity of $[\mathrm{Fe} / \mathrm{H}]<-4.0$ (Beers \& Christlieb 2005). In this subsection, we focus on two UMP stars with $[\mathrm{Fe} / \mathrm{H}]<-4.5$ : HE 0557-4840 (Norris et al. 2007) and SDSS J102915+172927 (Caffau et al. 2011a). The other UMP stars with $[\mathrm{Fe} / \mathrm{H}] \gtrsim-4.1$ are shown in $\S 3.2$ and $\S 3.3$

HE 0557-4840: The metallicity is $[\mathrm{Fe} / \mathrm{H}]=-4.77$ and the abundance ratios are shown in Figure 6a (Norris et al. 2007, 2012; Yong et al. 2013). The abundance pattern except for $[\mathrm{Cr} / \mathrm{Fe}]$ is well reproduced by an $\mathrm{SN}$ model with $E_{51}=5, M_{\mathrm{rem}}=5.57 \mathrm{M}_{\odot}$, and $M_{\mathrm{ej}}(\mathrm{Fe})=6.80 \times 10^{-3} \mathrm{M}_{\odot}$.

$\star[\mathbf{C r} / \mathbf{F e}]:[\mathrm{Cr} / \mathrm{Fe}]$ is overproduced even with taking into account the NLTE correction of +0.4 dex adopted in this paper. However, the NLTE correction for $[\mathrm{Cr} / \mathrm{Fe}]$ could be larger at lower $[\mathrm{Fe} / \mathrm{H}]$ (Bergemann \& Cescutti 2010) and improve the agreement.

SDSS J102915+172927: The metallicity is $[\mathrm{Fe} / \mathrm{H}]=$ -4.92 and the abundance ratios are shown in Figure 6b (Caffau et al. 2011a, 2012). This star is the most "metal"-deficient stars discovered so far. The abundance pattern is well reproduced by an SN model with $E_{51}=$ $20, M_{\mathrm{rem}}=3.03 \mathrm{M}_{\odot}$, and $M_{\mathrm{ej}}(\mathrm{Fe})=1.21 \times 10^{-1} \mathrm{M}_{\odot}$ (solid line). However, the number of measured abun- 


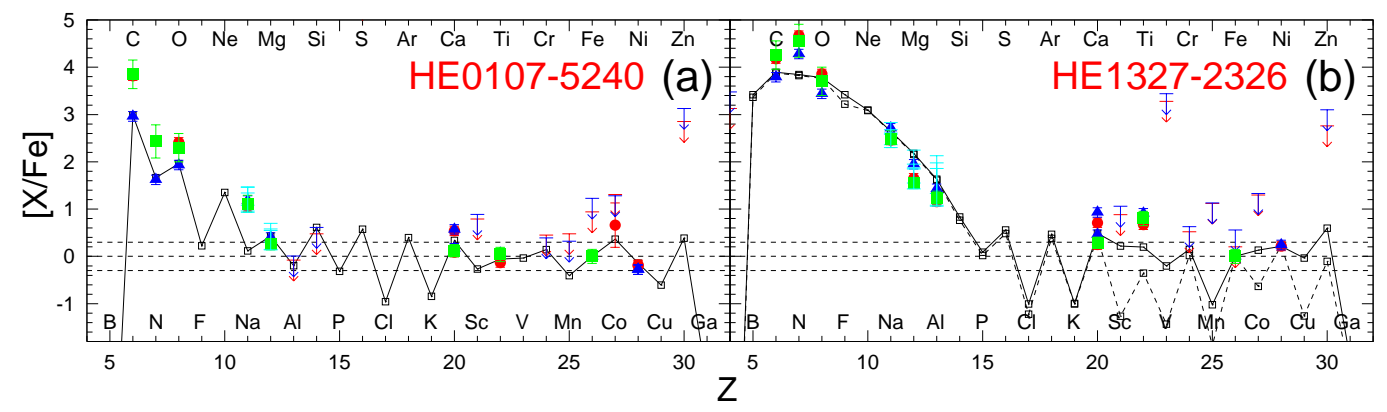

Fig. 7.- Same as Fig. 2 but for the HMP stars.

dance ratios are rather few. Thus the abundance pattern can be reproduced by a lower $E$ model with $E_{51}=5$, $M_{\mathrm{rem}}=3.49 \mathrm{M}_{\odot}$, and $M_{\mathrm{ej}}(\mathrm{Fe})=9.25 \times 10^{-2} \mathrm{M}_{\odot}$ as well (dashed line).

Although $[\mathrm{Fe} / \mathrm{H}]$ of the UMP stars are in between the EMP and HMP stars, metal abundance ratios of the UMP stars are similar to those of the EMP or CEMP stars. This indicates that the parent $\mathrm{SNe}$ are similar to those of the EMP or CEMP stars.

$[\mathrm{Fe} / \mathrm{H}]$ of EMP stars depends not only on the Fe mass ejected by an SN but also on the $\mathrm{H}$ mass which mixes with the SN ejecta. Although the $\mathrm{H}$ mass is determined mainly by the explosion energy in the 1D SNinduced star formation (Shigevama \& Tsuijmoto 1998; Thornton et al. (1998), sophisticated 3D simulations show that $[\mathrm{Fe} / \mathrm{H}]$ of a mixture gas are widely distributed from -5 to -1 with an average similar to the 1D calculations (Nakasato \& Shigevama 2000; Ritter et al. 2012). Therefore, we suggest that the UMP stars form from a cloud with lower $[\mathrm{Fe} / \mathrm{H}]$ which was enriched by the SN whose property was similar to SNe to originate the EMP or CEMP stars; this suggests that the EMP or CEMP stars also form around the same SN.

\subsection{Hyper Metal-Poor stars}

The most Fe-deficient stars are two HMP stars, HE 0107-5240 and HE 1327-2326. The HMP stars show similarly high $[\mathrm{C} / \mathrm{Fe}]$ but large differences in $[\mathrm{C} /(\mathrm{N}, \mathrm{O})]$ and $[(\mathrm{Na}, \mathrm{Mg}, \mathrm{Al}) / \mathrm{Fe}]$. The 5 mechanisms to enhance $[\mathrm{C} / \mathrm{Fe}]$ have been suggested and still under debate $(\S 3.2)$. Here, we adopt the faint SN mechanism which successfully explains the similarities and differences in the HMP stars (Umeda \& Nomoto 2003; Iwamoto et al. 2005; Tominaga et al. 2007a).

HE 0107-5240: The metallicity is $[\mathrm{Fe} / \mathrm{H}]=-5.61$ and the abundance ratios are shown in Figure $7 \mathrm{a}$ (Christlieb et al. 2002, 2004; Bessell \& Christlieb 2005; Collet et al. 2006; Yong et al. 2013). The abundance pattern except for $[\mathrm{Na} / \mathrm{Fe}]$ is well reproduced by an $\mathrm{SN}$ model with $E_{51}=5, M_{\mathrm{rem}}=6.24 \mathrm{M}_{\odot}$, and $M_{\mathrm{ej}}(\mathrm{Fe})=$ $8.02 \times 10^{-5} \mathrm{M}_{\odot}$.

$\star[\mathbf{C} / \mathbf{F e}]$ : Since the $3 \mathrm{D}$ effect considerably reduces $[\mathrm{C} / \mathrm{Fe}]$, the ejected $\mathrm{Fe}$ mass is increased by a factor of $\sim 5$ compared to the SN model in Iwamoto et al. (2005).

$\star$ [Co/Fe]: Although Umeda \& Nomoto (2003) and Iwamoto et al. (2005) attributed the abundance pattern of HE 0107-5240 to an SN with $E \leq 10^{51}$ ergs, such a low- $E$ explosion cannot reproduce high $[\mathrm{Co} / \mathrm{Fe}]$ of HE 0107-5240 (Bessell \& Christlieb 2005). Thus, assuming that large fallback takes place in an aspherical ex- plosion (Tominaga et al. 2007a), we adopt an energetic explosion model in which $[\mathrm{Co} / \mathrm{Fe}]$ is enhanced due to explosive nucleosynthesis in the high-entropy environment (Tominaga 2009).

$\star[\mathrm{Na} / \mathrm{Fe}]:$ The agreement of $[\mathrm{Na} / \mathrm{Fe}]$ could be improved with taking into account the NLTE effect, although the naive extrapolation of Andrievsky et al. (2007) enhances $[\mathrm{Na} / \mathrm{Fe}]$.

HE 1327-2326: The metallicity is $[\mathrm{Fe} / \mathrm{H}]=$ -5.88 and the abundance ratios are shown in Figure 7b (Frebel et al. 2005, 2008; Collet et al. 2006; Bonifacio et al. 2012; Yong et al. 2013). Some mixing processes might take place in $\mathrm{HE} 1327-2326$ since $\mathrm{Li}$ is not observed. Nevertheless, we assume that $\mathrm{N}$ is synthesized by the enhanced mixing in the SN progenitor because HE 1327-2326 is a subgiant star. The abundance pattern is well reproduced by an SN model with $E_{51}=5, M_{\mathrm{rem}}=5.72 \mathrm{M}_{\odot}$, and $M_{\mathrm{ej}}(\mathrm{Fe})=1.45 \times 10^{-5} \mathrm{M}_{\odot}$ (solid line).

$\star[\mathbf{N i} / \mathbf{F e}]:$ Frebel et al. (2008) reported an abundance ratio of $\mathrm{Ni}([\mathrm{Ni} / \mathrm{Fe}]=0.2)$, that is higher than $\mathrm{HE} 0107$ 5240. This suggests that a larger amount of matter with low $Y_{\mathrm{e}}$ is ejected in the parent SN of HE 1327-2326 than that of HE 0107-5240. Thus, the SN model of HE 13272326 has deeper $M_{\text {cut }}$ than that of HE 0107-5240.

* [Ti/Fe]: Contrary to HE 0107-5240, Co in HE 13272326 is not detected and thus the explosion energy of the parent SN of HE 1327-2326 is not well constrained. Although the high $[\mathrm{Ti} / \mathrm{Fe}]$ ratio is suggestive of high entropy, the enhancement of $[\mathrm{Ti} / \mathrm{Fe}]$ is not enough even with $E_{51}=5$. We note that [Ti II/Fe I] ratio could have an ambiguity due to a rather large NLTE effect (Bergemann 2011). If [Ti/Fe] is ignored, the abundance pattern of HE 1327-2326 can be explained by a low$E$ SN model with $E_{51}=0.72, M_{\text {rem }}=5.72 \mathrm{M}_{\odot}$, and $M_{\mathrm{ej}}(\mathrm{Fe})=1.53 \times 10^{-5} \mathrm{M}_{\odot}($ dashed line $)$.

\subsection{EMP stars in dwarf galaxies}

EMP stars with $[\mathrm{Fe} / \mathrm{H}] \lesssim-3.5$ have recently been discovered in dwarf galaxies and their detailed abundance ratios have become available. The abundance patterns of EMP stars in dwarf galaxies are similar to those of halo stars at low metallicity (e.g., Frebel et al. 2010), in contrast to the majority of stars in dwarf galaxies at $[\mathrm{Fe} / \mathrm{H}] \gtrsim-3$. Thus it is suggested that the early chemical enrichment of all galaxies may be nearly identical. Therefore, although the ISM density in dwarf galaxies at the formation of the EMP stars could be different, it is likely that the abundance pattern of the EMP star reflects dominantly nucleosynthesis in a single SN even 


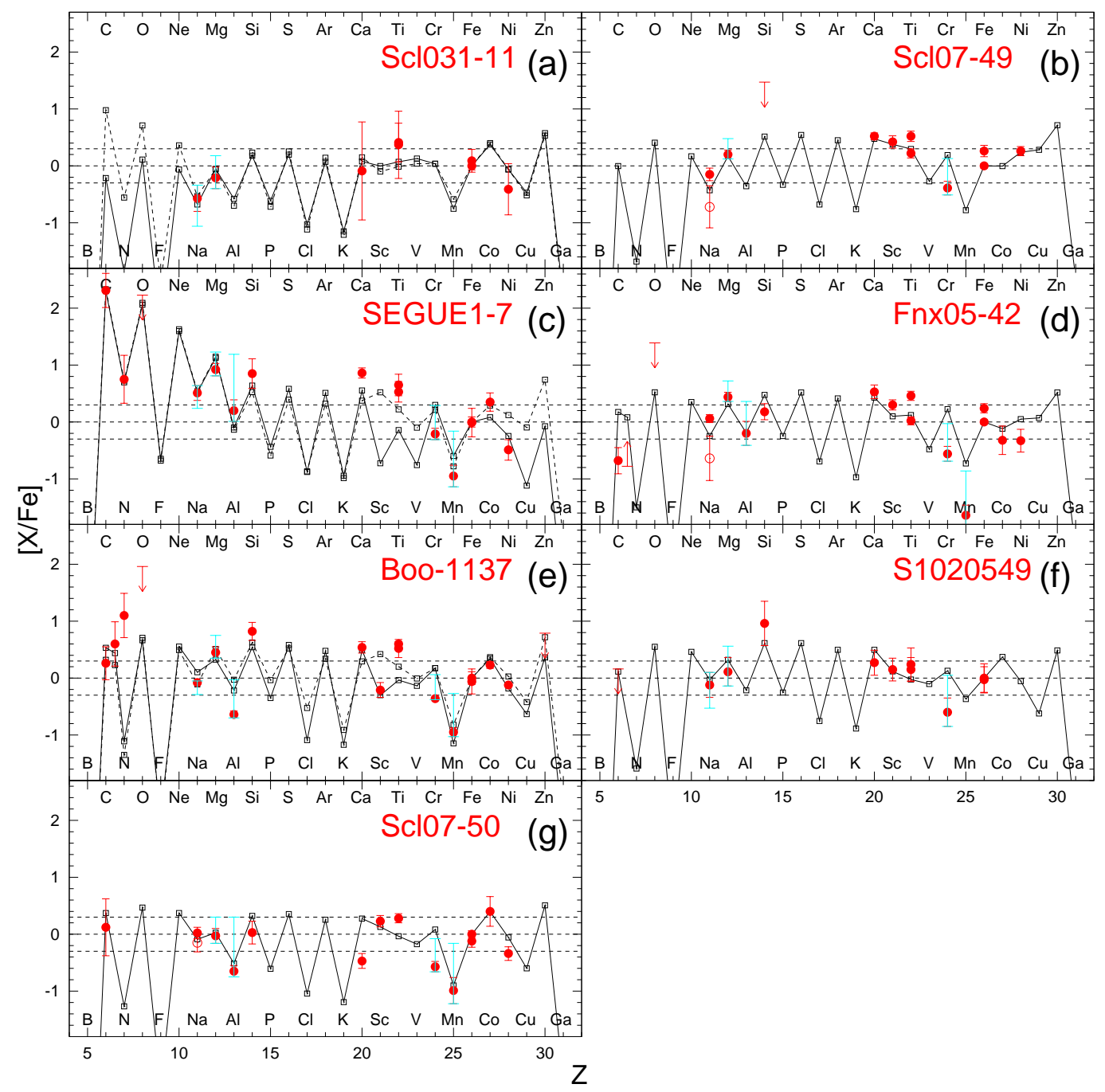

FIG. 8. - Same as Fig. 2 but for the EMP stars in dwarf galaxies.

in dwarf galaxies. Although, owing to the observational difficulty, the number of detectable lines is small and several abundance ratios of the EMP stars have large errors, we first construct Pop III SN models for the EMP stars in dwarf galaxies.

Scl 031-11: The metallicity is $[\mathrm{Fe} / \mathrm{H}]=-3.47$ and the abundance ratios are shown in Figure 8 a (Starkenburg et al. 2012). The abundance ratios $[(\mathrm{C}, \mathrm{N}, \mathrm{O}) / \mathrm{Fe}]$ are not available and error bars of $[(\mathrm{Ca}, \mathrm{Ti}) / \mathrm{Fe}]$ are large. The abundance pattern is well reproduced by an SN model with $E_{51}=20, M_{\text {rem }}=$ $3.26 \mathrm{M}_{\odot}$, and $M_{\mathrm{ej}}(\mathrm{Fe})=2.82 \times 10^{-1} \mathrm{M}_{\odot}$ (solid line).

$\star[\mathrm{Mg} / \mathbf{F e}]$ : The low $[\mathrm{Mg} / \mathrm{Fe}]$ can be explained without invoking the contribution of Type Ia supernovae but only with a hypernova-like core-collapse explosion. In the above model (solid line), the low $[\mathrm{Mg} / \mathrm{Fe}]$ is resulted from a large amount of $\mathrm{Fe}$ ejection which stems from large $E$ and relatively small $M_{\text {rem }}$. However, the constraints on $M_{\mathrm{ej}}(\mathrm{Fe})$ and $M_{\mathrm{rem}}$ is not strict owing to the small number of measured abundance ratios and the large errors. In fact, the abundance pattern could be explained by an SN model with $E_{51}=10, M_{\text {rem }}=5.82 \mathrm{M}_{\odot}$, and $M_{\mathrm{ej}}(\mathrm{Fe})=1.34 \times 10^{-2} \mathrm{M}_{\odot}$ as well (dashed line). In this model, much larger amount of fallback, i.e., large $M_{\text {mix }}$ and $f_{\mathrm{ej}}$, causes smaller amount of ejected $\mathrm{Mg}$, which leads to the lower $[\mathrm{Mg} / \mathrm{Fe}]$.

Scl 07-49: The metallicity is $[\mathrm{Fe} / \mathrm{H}]=-3.48$ and the abundance ratios are shown in Figure $8 \mathrm{~b}$ (Tafelmever et al. 2010). The abundance ratios $[(\mathrm{C}, \mathrm{N}, \mathrm{O}) / \mathrm{Fe}]$ are not available. The abundance pattern is well reproduced by an SN model with $E_{51}=20$, $M_{\mathrm{rem}}=2.63 \mathrm{M}_{\odot}$, and $M_{\mathrm{ej}}(\mathrm{Fe})=1.77 \times 10^{-1} \mathrm{M}_{\odot}$.

$\star[\mathrm{Mg} / \mathrm{Fe}]:$ The low $[\mathrm{Mg} / \mathrm{Fe}]$ is explained by large $M_{\mathrm{ej}}(\mathrm{Fe})$. However, as far as $E$ is high enough $\left(E_{51} \gtrsim 10\right)$ to realize high $[(\mathrm{Sc}, \mathrm{Ti}) / \mathrm{Fe}], M_{\mathrm{ej}}(\mathrm{Fe})$ is not well constrained as in the case of Scl 031-11.

SEGUE1-7: The metallicity is $[\mathrm{Fe} / \mathrm{H}]=-3.57$ and the abundance ratios are shown in Figure $8 \mathrm{c}$ (Norris et al. 2010a). We adopt the LTE Mn abundance without an increase of 0.4 dex. The abundance pattern except for $[\mathrm{Ti} / \mathrm{Fe}]$ is well reproduced by an $\mathrm{SN}$ model with $E_{51}=20, M_{\mathrm{rem}}=5.72 \mathrm{M}_{\odot}$, and $M_{\mathrm{ej}}(\mathrm{Fe})=$ $7.55 \times 10^{-4} \mathrm{M}_{\odot}$ (solid line). Since SEGUE1-7 is a CEMP star, the $\mathrm{SN}$ model with small $M_{\mathrm{ej}}(\mathrm{Fe})$ reproduces the abundance pattern as in $\S 3.2$.

$\star[(\mathbf{C a}, \mathbf{T i}, \mathbf{N i}) / \mathbf{F e}]$ : The high $[\mathrm{Ca} / \mathrm{Fe}]$ and low [Ni/Fe] implies a large $M_{\text {cut }}$ which leads to the underpro- 
duction of $[\mathrm{Ti} / \mathrm{Fe}]$. If we ignore $[\mathrm{Ni} / \mathrm{Fe}]$ determined by a single Ni I $\lambda 3858 \AA$ line, the agreements of $[\mathrm{Ti} / \mathrm{Fe}]$ and $[\mathrm{Co} / \mathrm{Fe}]$ are improved with an SN model with $E_{51}=20$, $M_{\mathrm{rem}}=5.72 \mathrm{M}_{\odot}$, and $M_{\mathrm{ej}}(\mathrm{Fe})=6.99 \times 10^{-4} \mathrm{M}_{\odot}($ dashed line).

Fnx 05-42: The metallicity is $[\mathrm{Fe} / \mathrm{H}]=-3.66$ and the abundance ratios are shown in Figure $8 \mathrm{~d}$ (Tafelmever et al. 2010). The abundance pattern is well reproduced by an SN model with $E_{51}=20, M_{\text {rem }}=$ $3.36 \mathrm{M}_{\odot}$, and $M_{\mathrm{ej}}(\mathrm{Fe})=1.18 \times 10^{-1} \mathrm{M}_{\odot}$. The low $[(\mathrm{Co}, \mathrm{Ni}) / \mathrm{Fe}]$ are reproduced by a large $M_{\text {cut }}$.

$\star[\mathbf{C} / \mathbf{F e}]$ : Fnx $05-42$ shows low $[\mathrm{C} / \mathrm{Fe}]$. The low $[\mathrm{C} / \mathrm{Mg}]$ is difficult to be reproduced by the SN model even with no fallback. However, since there are no constraint on the abundance of $\mathrm{N}$ and Fnx $05-42$ has low surface gravity, C could be converted to N in Fnx 05-42. We thus adopt a lower limit of $[(\mathrm{C}+\mathrm{N}) / \mathrm{Fe}]$ instead of $[\mathrm{C} / \mathrm{Fe}]$, which is consistent with the $\mathrm{SN}$ model.

Boo-1137: The metallicity is $[\mathrm{Fe} / \mathrm{H}]=-3.71$ and the abundance ratios are shown in Figure 8 (Norris et al. 2010b). Boo-1137 is an NEMP giant star and thus we adopt $[(\mathrm{C}+\mathrm{N}) / \mathrm{Fe}]$ instead of $[\mathrm{C} / \mathrm{Fe}]$ and $[\mathrm{N} / \mathrm{Fe}]$. We adopt the LTE Mn abundance without an increase of 0.4 dex. The abundance pattern except for $[\mathrm{Ti} / \mathrm{Fe}]$ is well reproduced by an SN model with $E_{51}=5, M_{\text {rem }}=$ $4.65 \mathrm{M}_{\odot}$, and $M_{\mathrm{ej}}(\mathrm{Fe})=4.49 \times 10^{-2} \mathrm{M}_{\odot}$ (solid line).

$\star[\mathbf{T i} / \mathbf{F e}]:$ The underproduction of $[\mathrm{Ti} / \mathrm{Fe}]$ stems from the low $[\mathrm{Sc} / \mathrm{Fe}]$. The disagreement of $[\mathrm{Ti} / \mathrm{Fe}]$ is improved with an $\mathrm{SN}$ model with higher $E$, i.e., $E_{51}=20$, $M_{\mathrm{rem}}=3.30 \mathrm{M}_{\odot}$, and $M_{\mathrm{ej}}(\mathrm{Fe})=8.29 \times 10^{-2} \mathrm{M}_{\odot}$ if we ignore $[\mathrm{Sc} / \mathrm{Fe}]$ determined by three lines (dashed line). The inability to simultaneously reproduce $[\mathrm{Sc} / \mathrm{Fe}]$ and $[\mathrm{Ti} / \mathrm{Fe}]$ might result from the NLTE effect of $[\mathrm{Ti} / \mathrm{Fe}]$ determined by Ti I lines because $[\mathrm{Sc} / \mathrm{Fe}]$ and $[\mathrm{Ti} / \mathrm{Fe}]$ in $\mathrm{Boo}-1137$ is incongruous with the correlation between $[\mathrm{Sc} / \mathrm{Fe}]$ and [Ti II/Fe] (Yong et al. 2013).

S 1020549: The metallicity is $[\mathrm{Fe} / \mathrm{H}]=-3.81$ and the abundance ratios are shown in Figure 8 ; (Frebel et al. 2010). For the CNO elements, only a low upper limit to $[\mathrm{C} / \mathrm{Fe}]$ is obtained. The abundance pattern is well reproduced by an SN model with $E_{51}=10, M_{\text {rem }}=$ $2.39 \mathrm{M}_{\odot}$, and $M_{\mathrm{ej}}(\mathrm{Fe})=1.43 \times 10^{-1} \mathrm{M}_{\odot}$. The low upper limit to $[\mathrm{C} / \mathrm{Fe}]$ and low $[\mathrm{Mg} / \mathrm{Fe}]$ are explained by large $M_{\mathrm{ej}}(\mathrm{Fe})$.

Scl 07-50: The metallicity is $[\mathrm{Fe} / \mathrm{H}]=-3.96$ and the abundance ratios are shown in Figure 8, (Tafelmeyer et al. 2010). The abundance pattern except for $[\mathrm{Ca} / \mathrm{Fe}]$ is well reproduced by an $\mathrm{SN}$ model with $E_{51}=10, M_{\mathrm{rem}}=4.75 \mathrm{M}_{\odot}$, and $M_{\mathrm{ej}}(\mathrm{Fe})=6.96 \times$ $10^{-2} \mathrm{M}_{\odot}$

$\star[\mathbf{C a} / \mathbf{F e}]$ : The observed abundance of $\mathrm{Ca}$ is suspiciously low due to the Ca I $\lambda 4227 \AA$ resonance line sensitive to an NLTE effect (Mashonkina et al. 2007; Tafelmever et al. 2010).

\section{LIMITATION OF ABUNDANCE PROFILING}

\subsection{Progenitor mass}

Although we expediently construct the SN models with the $25 \mathrm{M}_{\odot}$ progenitors, Pop III SNe with various progenitor masses should contribute to the chemical enrichment in the early Universe. In this subsection, we construct SN models for HE 1347-1025 (the EMP star), CS 29527015 and $\mathrm{BD}+44^{\circ} 493$ (the CEMP stars), HE 0557-4840 (the UMP stars), and HE 0107-5240 (the HMP stars) using the $40 \mathrm{M}_{\odot}$ progenitor without enhancement of mixing (Model C).

The $40 \mathrm{M}_{\odot} \mathrm{SN}$ models are also in reasonable agreement with the abundance patterns of the MP stars (Figs. 9a9e). The model parameters are summarized in Table 4 Improvements made in the $25 \mathrm{M}_{\odot} \mathrm{SN}$ models are also possible for the $40 \mathrm{M}_{\odot} \mathrm{SN}$ models as follows:

- The underproduction of $\mathrm{N}$ for $\mathrm{BD}+44^{\circ} 493$ and HE $0107-5240$ can be resolved if $10 \%$ and $2 \%$ of $\mathrm{C}$ in the He layer is converted to $\mathrm{N}$, respectively.

- The overproduction of $\mathrm{Cu}$ and $\mathrm{Zn}$ for $\mathrm{BD}+44^{\circ} 493$ can be improved by adopting larger $M_{\text {cut }}$, although it would make the agreement of $[(\mathrm{Ti}, \mathrm{Co}, \mathrm{Ni}) / \mathrm{Fe}]$ worse (dashed line).

The differences between the $25 \mathrm{M}_{\odot}$ and $40 \mathrm{M}_{\odot} \mathrm{SN}$ models, which can be used to distinguish the progenitor masses, appear in (1) $[\mathrm{C} / \mathrm{O}]$ for $\mathrm{HE}$ 1347-1025, (2) $[(\mathrm{Na}, \mathrm{Al}) / \mathrm{Mg}]$ for $\mathrm{HE} 1347-1025$, CS 29527-015, $\mathrm{BD}+44^{\circ} 493$, and $\mathrm{HE} 0557-4840$, and $(3)[\mathrm{Mg} / \mathrm{Fe}]$ for HE 0107-5240. Compared with the $25 \mathrm{M}_{\odot}$ models, the abundances of the $40 \mathrm{M}_{\odot} \mathrm{SN}$ model show the following features.

(1) Low $[\mathbf{C} / \mathrm{O}]$ : This stems from the fact that the $40 \mathrm{M}_{\odot}$ progenitor has a thicker $\mathrm{O}+\mathrm{Mg}$ layer compared to a $\mathrm{C}+\mathrm{O}$ layer than the $25 \mathrm{M}_{\odot}$ progenitor. The low $[\mathrm{C} / \mathrm{O}]$ is prominent in the $\mathrm{SN}$ model with small fallback, i.e., for the EMP stars. On the other hand, $[\mathrm{C} / \mathrm{O}]$ in the models for stars with high $[\mathrm{C} / \mathrm{Fe}]$ is similar to the $25 \mathrm{M}_{\odot} \mathrm{SN}$ model because the $\mathrm{O}$ mass is reduced by the large fallback of the $\mathrm{O}+\mathrm{Mg}$ layer. If the $\mathrm{O}$ abundance in HE 1347-1025 would be measured within the error of 0.2 dex, we could distinguish between the $25 \mathrm{M}_{\odot}$ and $40 \mathrm{M}_{\odot}$ progenitors.

(2) Low $[(\mathrm{Na}, \mathbf{A l}) / \mathrm{Mg}]$ : The $40 \mathrm{M}_{\odot}$ progenitor has lower $\mathrm{Na} / \mathrm{Mg}$ and $\mathrm{Al} / \mathrm{Mg}$ in the $\mathrm{O}+\mathrm{Mg}$ layer due to $\mathrm{C}$ burning at higher temperature than the $25 \mathrm{M}_{\odot}$ progenitor. Therefore, the $40 \mathrm{M}_{\odot} \mathrm{SN}$ models betterreproduce low $\left[\mathrm{Na} / \mathrm{Mg}\right.$ ] of $\mathrm{HE} 1347-1025, \mathrm{BD}+44^{\circ} 493$, and HE 0557-4840 (Figs. 9a, 9k, 9 $\mathrm{d}$ ) than the $25 \mathrm{M}_{\odot}$ SN models. The $40 \mathrm{M}_{\odot} \mathrm{SN}$ models also predict low $[\mathrm{Al} / \mathrm{Mg}$, which is consistent with the LTE abundance ratio but lower than the NLTE-corrected ratio. If the NLTE effect for $[\mathrm{Al} / \mathrm{Fe}]$ is small, the $40 \mathrm{M}_{\odot} \mathrm{SN}$ models are more favored for HE 1347-1025, BD+44 493 , and HE 0557-4840 than the $25 \mathrm{M}_{\odot} \mathrm{SN}$ models.

For CS $29527-015,[\mathrm{Na} / \mathrm{Mg}]$ of the $40 \mathrm{M}_{\odot} \mathrm{SN}$ model is not low enough to reproduce the observed ratio (Fig. 9b) and $[\mathrm{Na} / \mathrm{Mg}]$ of the $25 \mathrm{M}_{\odot} \mathrm{SN}$ model is clearly too high (Fig. 48). For $[\mathrm{Al} / \mathrm{Mg}]$ of CS 29527-015, on the other hand, both the $40 \mathrm{M}_{\odot}$ and $25 \mathrm{M}_{\odot} \mathrm{SN}$ models are consistent with the observation. Such consistency in $[\mathrm{Al} / \mathrm{Mg}]$ between both models and CS 29527-015 stems from the enhancement of ejection of explosively-synthesized $\mathrm{Al}$ in the $40 \mathrm{M}_{\odot} \mathrm{SN}$ model. This results from the high explosion energy and relatively small $M_{\text {mix }}$ that locates at the middle of $\mathrm{O}+\mathrm{Mg}$ layer.

(3) Low $[\mathrm{Mg} / \mathbf{F e}]$ : The $40 \mathrm{M}_{\odot} \mathrm{SN}$ model for HE $0107-$ 5240 shows lower $[\mathrm{Mg} / \mathrm{Fe}](\sim 0)$ than the $25 \mathrm{M}_{\odot} \mathrm{SN}$ model (Figs. 7t and 9p). Extended fallback reaching to the outer edge of the $\mathrm{O}+\mathrm{Mg}$ layer, i.e., large $M_{\text {mix }}$, is required to high $[\mathrm{C} / \mathrm{Mg}]$, while relatively small $M_{\text {cut }}$ 


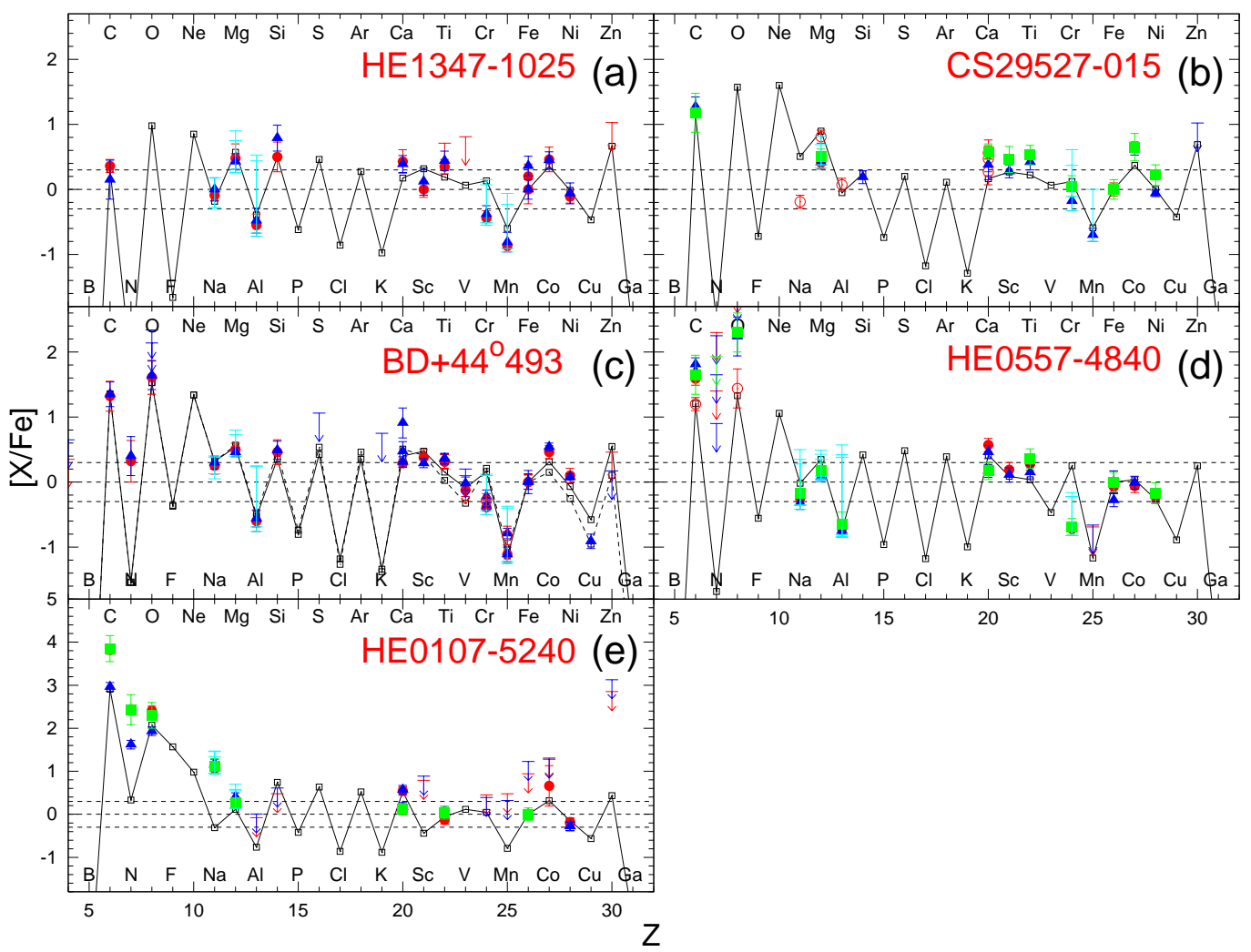

FIG. 9. - Same as Fig. 2 but with the $40 \mathrm{M}_{\odot}$ SN models. The properties and legends of Pop III SN models are summarized in Table 4

is required to reproduce low $[\mathrm{Si} / \mathrm{Fe}]$ and high $[\mathrm{Co} / \mathrm{Fe}]$. These requirements lead to lower $[\mathrm{Mg} / \mathrm{Fe}]$ of the $40 \mathrm{M}_{\odot}$ $\mathrm{SN}$ model than the observation. Thus, the $25 \mathrm{M}_{\odot} \mathrm{SN}$ model is favored for HE $0107-5240$.

The above examples demonstrate that it is possible to constrain the progenitor mass by using the abundance ratios $[\mathrm{C} / \mathrm{O}],[(\mathrm{Na}, \mathrm{Al}) / \mathrm{Mg}]$, and $[\mathrm{Mg} / \mathrm{Fe}]$. However, there are still theoretical and observational ambiguities. For example: (1) Mixing efficiency in the stellar evolution would affect the abundance ratios among the CNO elements. (2) The 3D and NLTE effects would affect the abundance determination. We emphasize that, in order to constrain the progenitor mass from the abundance pattern of each MP star, it is important to obtain the elemental abundances including 3D-NLTE effects as many as possible.

\subsection{Explosion energy}

In spherical explosion models, the temperature and entropy during explosive nucleosynthesis and the amount of fallback are determined predominantly by the explosion energy for a given progenitor model. This is one of the reason why the explosion energy is treated as an important property to rule explosive nucleosynthesis in previous studies. However, in multi-dimensional explosions, the temperature, entropy, and the amount of fallback can be different even for the same explosion energy or could be similar even for the different explosion energies. For example, the resultant SN yields can be different even with the same explosion energies (Tominaga et al. 2007a).

Figures $2 \mathrm{~d}$ and $3 \mathrm{~d}$ show that the SN models with $E_{51}=$ 5 and 20 explain the abundance patterns of CS 22897-
008 with the progenitor Model B and BS16467-062 with Model A (see also SDSS J102915+172927 in Figure 6b for the case with the small number of abundance determination). The abundance patterns of these SN models are similar, i.e., the $E$-dependence is small, except for $[\mathrm{F} / \mathrm{Fe}]$ in the progenitor Model B. As discussed for CS 29498-043 in $\S$ 3.2. $\mathrm{F}$ is enhanced via the hot CNO cycle ${ }^{14} \mathrm{~N}(p, \gamma){ }^{15} \mathrm{O}(\alpha, \gamma){ }^{19} \mathrm{Ne}\left(\beta^{+}\right){ }^{19} \mathrm{~F}$ during explosive nucleosynthesis if the N-rich layer experiences $T \gtrsim$ $7 \times 10^{8} \mathrm{~K}$ (e.g., Wiescher et al. 2010) ${ }^{12}$ Although NIR spectroscopy is required to determine the abundance of F (e.g., Smith et al. 2005), [F/Fe] could be an additional clue to constrain the explosion energy.

The weak constraint on the explosion energy stems from the fact that we allow arbitrary choices of the mixing-and-fallback parameters and the low-density modification. This is an inevitable shortcoming in the 1D model that approximates the aspherical explosion with the mixing-and-fallback model. In reality, the temperature, entropy, and fallback are determined by energy injection, geometry, e.g., a jet-opening angle, and fraction of kinetic energy in the jet-induced explosion (e.g., Tominaga 2009). Therefore, we leave the constraint on the explosion energy for future multi-dimensional studies.

12 The required temperature is higher than the value in Wiescher et al. (2010) due to the short cooling time scale in explosive nucleosynthesis. Explosive nucleosynthesis even at $T \lesssim 10^{9} \mathrm{~K}$ is important for synthesis of ${ }^{11} \mathrm{~B}$ and ${ }^{19} \mathrm{~F}$ in the He/N-rich layer of the H-envelope. 


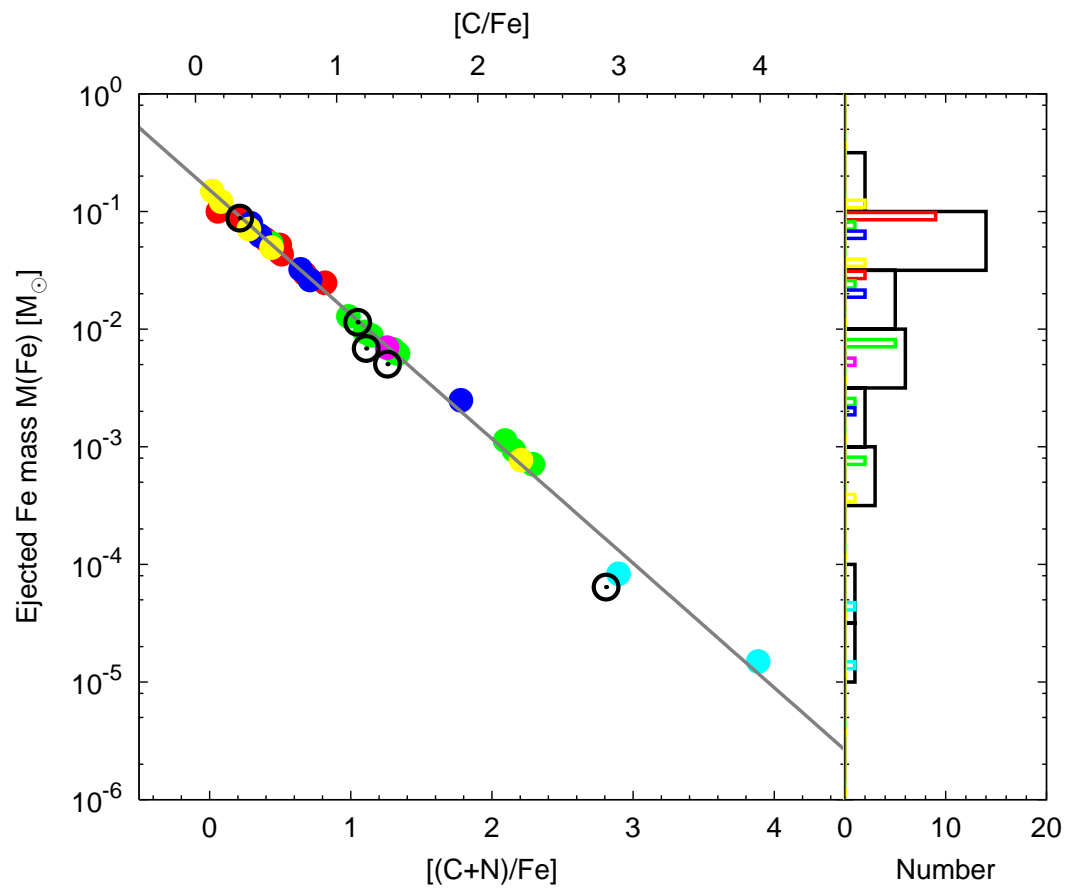

FIG. 10.- (Left) Ejected Fe masses of SN models for the individual MP stars as a function of $[(\mathrm{C}+\mathrm{N}) / \mathrm{Fe}]$. The color represents the EMP stars (red), the CEMP stars (green), the NEMP stars (blue), the UMP stars (magenta), the HMP stars (cyan), and the EMP stars in dwarf galaxies (yellow). The fitting curve [Eq. (2)] is also shown in gray line. The black open circles represent the $40 \mathrm{M}_{\odot} \mathrm{SN}_{\mathrm{N}}$ models shifted by a factor of $M_{\mathrm{pre}}(\mathrm{C}+\mathrm{N})_{25 \mathrm{M}_{\odot}} / M_{\mathrm{pre}}(\mathrm{C}+\mathrm{N})_{40 \mathrm{M}_{\odot}}$ according to Equation (2). (Right) Number distribution of ejected Fe masses of Pop III SNe. The colors represent the same as in the left panel but the black includes $25 \mathrm{M}_{\odot} \mathrm{SN}$ models for all kinds of MP stars.

\section{DISTRIBUTION OF SUPERNOVA PROPERTIES}

According to the Pop III SN models constructed in the previous sections, we investigate how properties of Pop III SNe link to the abundance ratios. In order to reduce the uncertainties due to the lack of observations, we focus on the 34 Pop III SN models reproducing the EMP stars with determined abundance ratios or strict upper limit of 4 elemental groups listed in $\S 3$, except for HE 0057$5959 .{ }^{13}$ The adopted models are indicated by "Yes" in Table 3. We employ theoretical abundance ratios instead of observed abundance ratios to reduce ambiguities due to 3D-NLTE effects.

The following subsections proceed as follows.

First, we derive the relation between the properties of observationally-selected theoretical models and the abundance ratios of their yields. The relation itself is free from the observational uncertainties such as 3D-NLTE effects, although the requirements to the SN models from the EMP stars could be changed by 3D-NLTE effects.

Second, we derive the distribution of properties of Pop III SNe. The observational uncertainties and poor reproduction by theoretical models influence the distribution of properties. In addition to these limitations, the distribution also involves the following assumptions; (1) the detection of EMP stars at $[\mathrm{Fe} / \mathrm{H}] \lesssim-3.5$ is observationally unbiased and (2) all Pop III SNe contribute to the EMP stars at $[\mathrm{Fe} / \mathrm{H}] \lesssim-3.5$ with the same probability. We discuss validity of these assumption in $\S 5.3$.

13 HE 0057-5959 could be originated from the mass transfer from the N-rich AGB companion.

\section{1. $[(C+N) / F e]$ vs. $M_{\mathrm{ej}}(\mathrm{Fe})$}

The ejected Fe mass $M_{\mathrm{ej}}(\mathrm{Fe})$ is an important property of the SN because energy release from the radioactive decay of parent ${ }^{56} \mathrm{Ni}$ powers the light curve of the $\mathrm{SN} .[(\mathrm{C}+\mathrm{N}) / \mathrm{Fe}]$ of MP stars constrain the ratio between $M_{\mathrm{ej}}(\mathrm{Fe})$ and the ejected mass of $\mathrm{C}+\mathrm{N}, M_{\mathrm{ej}}(\mathrm{C}+\mathrm{N})$, of Pop III SNe. Since some fractions of $\mathrm{C}$ and $\mathrm{N}$ are destroyed by explosive nucleosynthesis and fall back onto the central remnant, $M_{\mathrm{ej}}(\mathrm{C}+\mathrm{N})$ is reduced from the mass of $\mathrm{C}+\mathrm{N}$ in the $\mathrm{SN}$ progenitor $\left[M_{\mathrm{pre}}(\mathrm{C}+\mathrm{N})\right]$ and such a reduction occurs more efficiently for the SN models with larger amount of fallback.

The left panel of Figure [10 demonstrates that $[(\mathrm{C}+\mathrm{N}) / \mathrm{Fe}]$ relates to $M_{\mathrm{ej}}(\mathrm{Fe})$. The relation is well fitted by the following equation

$$
\begin{gathered}
M_{\mathrm{ej}}(\mathrm{Fe})=0.931\left(\frac{X_{\mathrm{Fe}}}{X_{\mathrm{C}}+X_{\mathrm{N}}}\right)_{\odot} \\
\times M_{\text {pre }}(\mathrm{C}+\mathrm{N}) \times 10^{-1.058[(\mathrm{C}+\mathrm{N}) / \mathrm{Fe}]},
\end{gathered}
$$

where $X_{\mathrm{Fe}}, \quad X_{\mathrm{C}}$, and $X_{\mathrm{N}}$ are the mass fractions of $\mathrm{Fe}, \mathrm{C}$, and $\mathrm{N},{ }^{14}$ respectively. The ratio $M_{\mathrm{ej}}(\mathrm{C}+\mathrm{N}) / M_{\mathrm{pre}}(\mathrm{C}+\mathrm{N})$ ranges over $0.545-$ 0.931 for $[(\mathrm{C}+\mathrm{N}) / \mathrm{Fe}]=-4$ to 0 . We note that $[\mathrm{C} / \mathrm{Fe}] \simeq[(\mathrm{C}+\mathrm{N}) / \mathrm{Fe}]+0.1$ for EMP stars without $\mathrm{N}$ enhancement (Asplund et al. 2009).

$M_{\text {pre }}(\mathrm{C}+\mathrm{N})$ depends on $M_{\mathrm{MS}}$ of the SN progenitor. For example, among the models in Umeda \& Nomoto (2005) (see also Tominaga et al. 2007b), $M_{\text {pre }}(\mathrm{C}+\mathrm{N})=$ $0.07 \mathrm{M}_{\odot}$ and $1.8 \mathrm{M}_{\odot}$ for the $13 \mathrm{M}_{\odot}$ and $50 \mathrm{M}_{\odot}$ progenitors, respectively. The $40 \mathrm{M}_{\odot}$ models constructed in $\S 4.1$ are

$$
14\left(\frac{X_{\mathrm{Fe}}}{X_{\mathrm{C}}+X_{\mathrm{N}}}\right)_{\odot}=0.423 \text { Asplund et al. 2009). }
$$




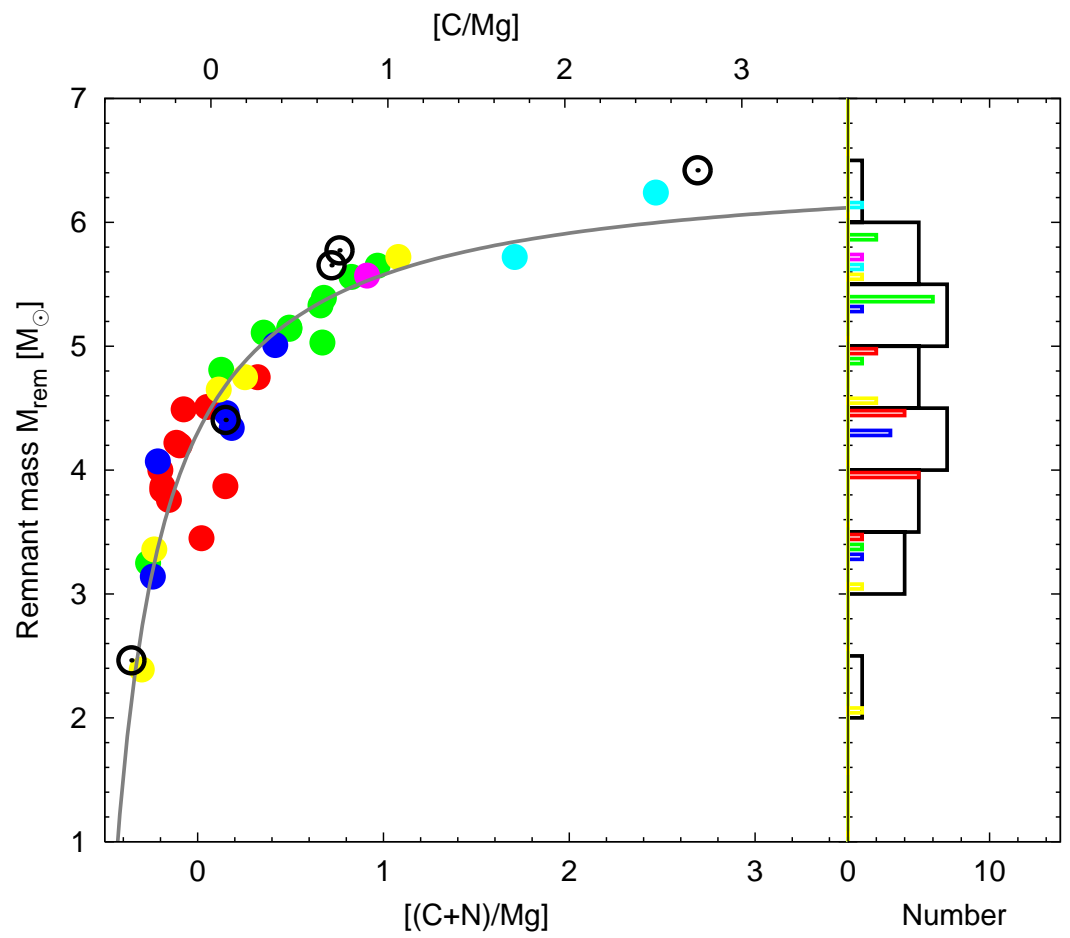

FIG. 11.- (Left) Remnant masses of SN models for the individual MP stars as a function of $[(\mathrm{C}+\mathrm{N}) / \mathrm{Mg}]$. The color represents the same as in the left panel of Figure 10, The fitting curve [Eq. (3)] is also shown in gray line. The black open circles represent the 40M $\odot$ models shifted by a factor of $M_{\mathrm{C}+\mathrm{O}, 25 \mathrm{M}_{\odot}} / M_{\mathrm{C}+\mathrm{O}, 40 \mathrm{M}_{\odot}}$ according to Equation (3). (Right) Number distribution of remnant masses of Pop III SNe. The colors represent the same as in the right panel of Figure 10

also shown in the left panel of Figure 10, shifting by a factor of $M_{\text {pre }}(\mathrm{C}+\mathrm{N})_{25 \mathrm{M}_{\odot}} / M_{\text {pre }}(\mathrm{C}+\mathrm{N})_{40 \mathrm{M}_{\odot}}(=0.592)$ according to Equation (2), where $M_{\text {pre }}(\mathrm{C}+\mathrm{N})_{25 \mathrm{M}_{\odot}}(=$ $\left.0.388 \mathrm{M}_{\odot}\right)$ and $M_{\text {pre }}(\mathrm{C}+\mathrm{N})_{40 \mathrm{M}_{\odot}}\left(=0.656 \mathrm{M}_{\odot}\right)$ are the mass of $\mathrm{C}+\mathrm{N}$ in the $25 \mathrm{M}_{\odot}$ and $40 \mathrm{M}_{\odot}$ progenitors, respectively. The $40 \mathrm{M}_{\odot} \mathrm{SN}$ models are consistent with the $25 \mathrm{M}_{\odot}$ SN models. The variation of $M_{\text {pre }}(\mathrm{C}+\mathrm{N})$ in the progenitor models with $M_{\mathrm{MS}}=13-50 \mathrm{M}_{\odot}(\sim 1.5 \mathrm{dex})$ is smaller than that of $[(\mathrm{C}+\mathrm{N}) / \mathrm{Fe}]$ in the EMP stars $(\sim 4 \mathrm{dex})$. Therefore, the real variation of $M_{\mathrm{ej}}(\mathrm{Fe})$ amounts at least to $\sim 2.5$ dex, which would reflect the differences in the explosion geometry, explosion energy, and fallback.

We find from Figure 10 (right) that the most common Pop III SN yields $M_{\mathrm{ej}}(\mathrm{Fe})=3 \times 10^{-2}-10^{-1} \mathrm{M}_{\odot}$, which corresponds to the peak bolometric magnitude of Type Ibc SNe $M_{\text {peak }}^{\text {bol }}$ of -16.6 to -17.4 mag. ${ }^{15}$ This is consistent with the brightness of the most common Type Ibc SN in the present day (e.g., $M_{\text {peak }}^{\text {bol }} \sim-17.5 \mathrm{mag}$, Richardson et al. 2002; see also Li et al. 2011 in which host galaxy extinction is not corrected).

On the other hand, the number distribution of $M_{\mathrm{ej}}(\mathrm{Fe})$ in the right panel of Figure 10 indicates an extended tail for Pop III SNe down to $M_{\mathrm{ej}}(\mathrm{Fe}) \sim 10^{-2}-10^{-5} \mathrm{M}_{\odot} \cdot{ }^{16}$ Such a tail corresponds to Type Ibc SNe with $M_{\text {peak }}^{\text {bol }}>$ $-14.9 \mathrm{mag}$, which is $2 \mathrm{mag}$ fainter than the most com-

15 The peak brightness of a Type Ibc SN is roughly proportional to $M_{\mathrm{ej}}(\mathrm{Fe})$. A Type Ibc SN with $M_{\mathrm{ej}}(\mathrm{Fe})=0.07 \mathrm{M}_{\odot}$ has $M_{\text {peak }}^{\text {bol }}$ -17 mag (e.g., SN 2008D, Tanaka et al. 2009) independent of the metallicity.

16 The tail would be more extended if the fallback reaches the He layer that contains $\mathrm{C}$ and $\mathrm{N}$. mon Type Ibc SNe in the present day. Such faint Type Ibc SNe (e.g., SN 2008ha, Valenti et al. 2009; Folev et al. 2010) and GRBs without associated SNe (e.g., GRB 060614, Fvnbo et al. 2006) have been discovered in the nearby Universe. However, the fraction of such faint Type Ibc SNe is very small in the present day, in contrast to a non-negligible fraction of Pop III SNe with $M_{\mathrm{ej}}(\mathrm{Fe}) \lesssim 10^{-2} \mathrm{M}_{\odot}$. Such a discrepancy in the fractions could stem from the following biases; (1) There exists an observational bias in the nearby Universe due to the dimness of faint Type Ibc SNe. The fraction of dim $\mathrm{SNe}$ in the nearby Universe is an interesting issue to be addressed (e.g., Horiuchi et al. 2011). (2) There may be a bias that the EMP stars are enriched from SNe of massive stars while nearby SNe are mainly the explosions of less-massive stars.

Future nearby SN surveys and observations will solve these biases with deep observations of nearby $\mathrm{SNe}$ and the increase of the number of nearby $\mathrm{SNe}$ of massive stars. If the discrepancy in fraction is real, it implies that the Pop III SNe tend to have smaller $M_{\mathrm{ej}}(\mathrm{Fe})$ than the present day SNe. This could be explained by the larger fallback in Pop III SNe which is led by an existence of thicker $\mathrm{H}$ envelope due to the weaker mass loss and a more compact structure due to the lower opacity in a Pop III star.

\section{2. $[(C+N) / M g]$ vs. $M_{\text {rem }}$}

The remnant mass $M_{\text {rem }}$ of the $\mathrm{SN}$ model is determined by Equation (1). $M_{\text {rem }}$ is larger for higher $[(\mathrm{C}+\mathrm{N}) / \mathrm{Mg}]$ because high $[(\mathrm{C}+\mathrm{N}) / \mathrm{Mg}]$ requires large $M_{\text {mix }}$ (being close to the outer boundary of the $\mathrm{C}+\mathrm{O}$ core) and small $f_{\text {ej }}$. The relation between $M_{\text {rem }}$ and $[(\mathrm{C}+\mathrm{N}) / \mathrm{Mg}]$ is 
shown in the left panel of Figure [11. The relation is well fitted by the following formula

$$
M_{\mathrm{rem}}=M_{\mathrm{C}+\mathrm{O}}\left(1-\frac{0.241}{[(\mathrm{C}+\mathrm{N}) / \mathrm{Mg}]+0.716}\right),
$$

where $M_{\mathrm{C}+\mathrm{O}}$ is the $\mathrm{C}+\mathrm{O}$ core mass. The $M_{\mathrm{C}+\mathrm{O}}$ (and thus resulting $M_{\mathrm{rem}}$ ) depends on the progenitor mass, e.g., $M_{\mathrm{C}+\mathrm{O}, 13 \mathrm{M}_{\odot}}=2.4 \mathrm{M}_{\odot}, M_{\mathrm{C}+\mathrm{O}, 25 \mathrm{M}_{\odot}}=6.49 \mathrm{M}_{\odot}$, $M_{\mathrm{C}+\mathrm{O}, 40 \mathrm{M}_{\odot}}=14.0 \mathrm{M}_{\odot}$, and $M_{\mathrm{C}+\mathrm{O}, 50 \mathrm{M}_{\odot}}=18.3 \mathrm{M}_{\odot}$, for the progenitors of $13 \mathrm{M}_{\odot}, 25 \mathrm{M}_{\odot}, 40 \mathrm{M}_{\odot}$, and $50 \mathrm{M}_{\odot}$, respectively. The results for the $40 \mathrm{M}_{\odot}$ SN models constructed in $\S 4.1$ are also shown in the left panel of Fig-

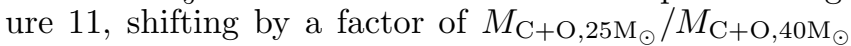
$(=0.462)$ according to Equation (3). The relation for the $40 \mathrm{M}_{\odot} \mathrm{SN}$ models is consistent with that for the $25 \mathrm{M}_{\odot}$ SN models. The parent Pop III SNe of the EMP stars with high $[\mathrm{C} / \mathrm{Mg}](\gtrsim+3)$ are black-hole-forming ${ }^{17} \mathrm{SNe}$ with $M_{\text {rem }} \sim 2.4 \mathrm{M}_{\odot}$ for the $13 \mathrm{M}_{\odot}$ star and $\sim 18.3 \mathrm{M}_{\odot}$ for the $50 \mathrm{M}_{\odot}$ star.

According to the $25 \mathrm{M}_{\odot} \mathrm{SN}$ models, $M_{\text {rem }}$ widely distributes from $3 \mathrm{M}_{\odot}$ to $6 \mathrm{M}_{\odot} \cdot{ }^{18}$ Although it is difficult to constrain $M_{\text {rem }}$ from the observations of nearby SNe because of the uncertainty in the fallback mass, the mass distribution of stellar-mass black hole is constrained from the observations of X-ray binary systems. The width of $M_{\text {rem }}$ distribution is similar to that of the present day mass distribution of stellar-mass black holes Ozel et al. 2010; Farr et al. 2011). We note that the distribution of $M_{\text {rem }}$ is extended depending on the initial mass function of Pop III stars. Further investigation including an integration over the initial mass function is required to quantitatively evaluate the consistency between the distribution of $M_{\text {rem }}$ of Pop III SNe and present day stellarmass black hole masses.

\subsection{Validity of assumptions}

The above methods stand on the assumptions: (1) the detection of EMP stars at $[\mathrm{Fe} / \mathrm{H}] \lesssim-3.5$ is unbiased toward any subclasses categorized in this paper and (2) all Pop III SNe contribute to any subclasses of EMP stars with $[\mathrm{Fe} / \mathrm{H}] \lesssim-3.5$ at the same possibility.

The assumption (1) is appropriate because the MP star surveys are performed with $\mathrm{Ca}$ lines not with e.g., $\mathrm{CH}$ or $\mathrm{NH}$ line, and follow-up spectroscopic observations are complete at $[\mathrm{Fe} / \mathrm{H}] \lesssim-3.5$. The assumption (2) could be of no matter if the mass of gas enriched by Pop III SNe and the initial mass function of the next-generation stars do not depend on the metallicity at $[\mathrm{Fe} / \mathrm{H}] \lesssim-3.5$. The $3 \mathrm{D}$ computations propose wide distribution of $[\mathrm{Fe} / \mathrm{H}]$ from -5 to -1 but the gas mass seems to slowly increase as a function of metallicity (Ritter et al. 2012). ${ }^{19}$ The dependence of initial mass function on metallicity is under investigation (e.g., Omukai et al. 2005; Dopcke et al. 2012). Furthermore the fragmentation mechanisms of clouds are still under

17 It depends on the maximum mass of a neutron star (e.g., Demorest et al. 2010)

18 The maximum $M_{\text {rem }}$ would be larger if the materials in the He layer fall back.

19 The 3D results depend on the details of simulations, and it is required to confirm the dependence of gas metallicity on properties, e.g., explosion energy, of Pop III SNe with systematic works. debate (fine-structure cooling, Bromm \& Loeb 2003 and dust-induced cooling, Schneider et al. 2006) and it is recently suggested that low-mass stars can form even at $Z=0$ by disk fragmentation (Clark et al. 2011; Susa 2013). Convolving the metallicity distribution of gas mass and the initial mass function with Equations (2) and (3) gives realistic distribution of properties of Pop III SNe through the comparison with the distribution of the abundance ratios of the EMP stars.

\section{DISCUSSION}

The SN models well reproduce the abundance patterns of most of EMP stars. However, there are several difficult elements.

(1) The high $[(\mathrm{Sc}, \mathrm{Ti}) / \mathrm{Fe}]$ of the EMP stars require the high-entropy environment in the explosion. The variation of $f_{\rho}$ implies that Pop III SNe are aspherical explosions with different energy deposition rate and/or jet-opening angle (Tominaga 2009).

(2) Although the $Y_{\mathrm{e}}$ profile is modified referring only $[(\mathrm{Mn}, \mathrm{Co}) / \mathrm{Fe}]$ in this paper, the abundance ratios of elements synthesized in the complete Si-burning layer could minutely constrain the $Y_{\mathrm{e}}$ distribution in the innermost layer of the SN ejecta.

(3) The ratio $[\mathrm{K} / \mathrm{Fe}]$ of the present $\mathrm{SN}$ models are not in agreement with $[\mathrm{K} / \mathrm{Fe}]$ of all the EMP stars. Although the NLTE effect on $[\mathrm{K} / \mathrm{Fe}\rceil$ is large at $[\mathrm{Fe} / \mathrm{H}] \sim-2$ to -1 (Takeda et al. 2002; Zhang et al. 2006), the effect at low metallicity is not large enough to reconcile the discrepancy. There are several suggestions to enhance $[\mathrm{K} / \mathrm{Fe}]$. The underproduction of $\mathrm{K}$ could be solved by nucleosynthesis in $p$-rich ejecta which may be led by neutrino process (Iwamoto et al. 2006).

We leave these issues in future investigation.

The determination of the abundance ratios in the EMP stars is suffered from the 3D-NLTE effects and the difficult determination of the surface gravity. An impact on the abundance ratios varies depending on individual stars. The uncertainties of the abundance ratios make it difficult to minutely constrain properties of SN models. For example, the uncertainties for $\mathrm{HE} 1300+0157$ are as large as $\sim 2.0$ dex for $[(\mathrm{C}, \mathrm{O}) / \mathrm{Fe}]$ and $\sim 1.0 \mathrm{dex}$ for $[(\mathrm{Na}, \mathrm{Mg}, \mathrm{Al}) / \mathrm{Fe}]$. Such large uncertainties lead large ambiguities of $M_{\mathrm{ej}}(\mathrm{Fe})$ by two orders of magnitude and $M_{\text {rem }}$ by a factor of $\sim 2.5$. The low $[\alpha / \mathrm{Fe}]$ for the surface gravity of a dwarf star can be reproduced even without the mixing-and-fallback process.

We find that the progenitor mass can be constrained by the abundance ratios between the elements synthesized in the same layer. In particular, the accurate determination of the abundance ratios among $\mathrm{Na}, \mathrm{Mg}$, and $\mathrm{Al}$ could contribute to the determination of the progenitor mass. We also indicate that the abundance of $F$ is useful for the constraint on $E$ if $\mathrm{N}$ is enhanced in the progenitor and the N-rich layer experiences high temperature $\left(T \gtrsim 7 \times 10^{8} \mathrm{~K}\right)$ during the shock propagation. However, we caution that it is still difficult to uniquely determine the progenitor mass and explosion energy with arbitrary choice of the mixing-and-fallback parameters because supernova nucleosynthesis also depends on the explosion 


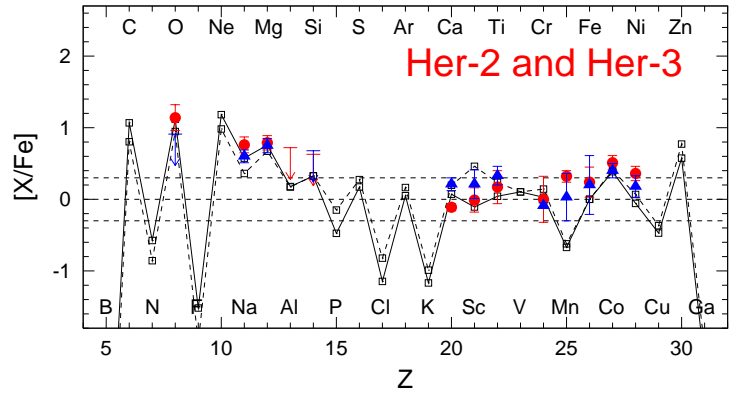

Fig. 12.- Same as Fig. 2 but for the stars in Hercules dwarf galaxy. The properties and legends of Pop III SN models are summarized in Table 3

geometry. 2D or 3D explosion simulations tie the explosion geometry, the explosion energy, and the mainsequence mass, and help deriving physical insight on the central engine. Furthermore, the abundance ratios in the progenitor strongly depends on mixing processes in the SN progenitor, which are required to be dealt with $3 \mathrm{D}$ computation (e.g., Meakin \& Arnett 2007). It is important to adopt SN models with attention to these caveats.

The number fraction of stars with low $[(\mathrm{C}+\mathrm{N}) / \mathrm{Fe}]$ in dwarf galaxies is larger than that in Galactic halo and thus $M_{\mathrm{ej}}(\mathrm{Fe})$ for the EMP stars in dwarf galaxies is distributed to higher values than those in halo stars. We note that the smaller fraction of the EMP stars with high $[(\mathrm{C}+\mathrm{N}) / \mathrm{Fe}]$ in dwarf galaxies is not explained by the observational bias. However, there are caveats that the number of EMP stars with $[\mathrm{Fe} / \mathrm{H}] \lesssim-3.5$ in dwarf galaxies are still small and that the abundance of $\mathrm{N}$ is not well constrained except for two stars. Although this might stem from statistical scatter, if real, the Pop III $\mathrm{SNe}$ in dwarf galaxies yield low $[\alpha / \mathrm{Fe}]$ which is consistent with the MP stars in dwarf galaxies at $[\mathrm{Fe} / \mathrm{H}] \gtrsim-3$. This would be verified by future studies with increasing the number of EMP stars in dwarf galaxies.

The chemical enrichment in dwarf galaxies is suggested to be inhomogeneous even at higher metallicity $[\mathrm{Fe} / \mathrm{H}] \gtrsim-3$ (e.g., Koch et al. 2008; Venn et al. 2012) because the abundance ratios of MP stars in dwarf galaxies show large dispersion. The abundance patterns of stars formed in the inhomogeneous environment can reflect predominantly nucleosynthesis in a single SN even if the metallicity is high. Indeed, the unusual abundance patterns of Her-2 and Her-3 stars with $[\mathrm{Fe} / \mathrm{H}]=-2$ are reproduced by Pop III SN models with high explosion energies (Fig. 12). If the inhomogeneity of ISM is held at high metallicity, a larger number of stars can be applied to constrain the properties of Pop III SNe in the dwarf galaxies. It is important to find out the transition period from inhomogeneous to homogeneous metal enrichment in dwarf galaxies.

\section{CONCLUDING REMARKS}

We construct the Pop III SN models reproducing the abundance patterns of the 48 EMP stars with $[\mathrm{Fe} / \mathrm{H}] \lesssim$ -3.5 in the Galactic halo and dwarf galaxies (abundance profiling). We adopt the spherical explosion models in which the aspherical effects are taken into account as mixing-and-fallback. According to the SN models, we reveal the correlations between the abundance ratios and the properties of Pop III SNe and derive the fitting formulae of the relations: $[(\mathrm{C}+\mathrm{N}) / \mathrm{Fe}]$ vs. $M_{\mathrm{ej}}(\mathrm{Fe})$, and $[(\mathrm{C}+\mathrm{N}) / \mathrm{Mg}]$ vs. $M_{\text {rem }}$. This is enabled by the construction of Pop III SN models for numerous EMP stars which is impossible to be executed with $2 \mathrm{D}$ or $3 \mathrm{D}$ simulations because of computational costs. With the use of these relations, the distribution of the abundance ratios of the EMP stars is projected to the distribution of $M_{\mathrm{ej}}(\mathrm{Fe})$ and $M_{\text {rem }}$ of Pop III SNe. These clarify that the distribution of $M_{\mathrm{ej}}(\mathrm{Fe})$ extends down to $M_{\mathrm{ej}}(\mathrm{Fe}) \sim 10^{-5} \mathrm{M}_{\odot}$, which might imply that the Pop III SNe tend to have smaller $M_{\mathrm{ej}}(\mathrm{Fe})$ than the present day $\mathrm{SNe}$, and that the distribution of $M_{\text {rem }}$ is as wide as $3 \mathrm{M}_{\odot}$ which is consistent with the mass distribution of present day stellar-mass black holes.

These demonstrate that the abundance profiling is a powerful tool to derive the properties of Pop III SNe. The future surveys and follow-up observations can increase the number of EMP stars at $[\mathrm{Fe} / \mathrm{H}] \lesssim-3.5$ and make it possible to more rigorously constrain the properties of Pop III SNe. We emphasize the importance of the precise determination of the abundance ratios including the 3D-NLTE effects and the stellar parameters. These are essential to constrain the properties of Pop III SNe including $E$ and $M_{\mathrm{MS}}$ from the abundance pattern of individual EMP stars by abundance profiling.

The authors thank Chiaki Kobayashi, Hajime Susa, and Marco Limongi for fruitful discussion on chemical abundance, star formation in the early Universe, and synthesis of $\mathrm{F}$, respectively. Data analysis were in part carried out on the general-purpose PC farm at Center for Computational Astrophysics, National Astronomical Observatory of Japan. This research has been supported in part by World Premier International Research Center Initiative, MEXT, Japan, and by the Grant-in-Aid for Scientific Research of the JSPS (20540226, 21840055, 23224004, 23740157) and MEXT (19047004, 22012003).

\section{REFERENCES}

Andrievsky, S. M., Spite, M., Korotin, S. A., Spite, F., Bonifacio, P., Cayrel, R., François, P., \& Hill, V. 2010, A\&A, 509, A88 Andrievsky, S. M., Spite, M., Korotin, S. A., Spite, F., Bonifacio, P., Cayrel, R., Hill, V., \& François, P. 2007, A\&A, 464, 1081 . 2008, A\&A, 481, 481

Aoki, W., Barklem, P. S., Beers, T. C., Christlieb, N., Inoue, S. García Pérez, A. E., Norris, J. E., \& Carollo, D. 2009, ApJ, 698, 1803

Aoki, W., Norris, J. E., Ryan, S. G., Beers, T. C., Christlieb, N., Tsangarides, S., \& Ando, H. 2004, ApJ, 608, 971
Argast, D., Samland, M., Gerhard, O. E., \& Thielemann, F. 2000, A\&A, 356, 873

Asplund, M. 2005, ARA\&A, 43, 481

Asplund, M., Grevesse, N., Sauval, A. J., \& Scott, P. 2009, ARA\&A, 47, 481

Audouze, J., \& Silk, J. 1995, ApJ, 451, L49

Barklem, P. S. et al. 2005, A\&A, 439, 129

Beers, T. C., \& Christlieb, N. 2005, ARA\&A, 43, 531

Beers, T. C., Preston, G. W., \& Shectman, S. A. 1992, AJ, 103, 1987 
Beers, T. C., Rossi, S., Norris, J. E., Ryan, S. G., \& Shefler, T. 1999, AJ, 117, 981

Beers, T. C., Sivarani, T., Marsteller, B., Lee, Y., Rossi, S., \& Plez, B. 2007, AJ, 133, 1193

Bergemann, M. 2011, MNRAS, 413, 2184

Bergemann, M., \& Cescutti, G. 2010, A\&A, 522, A9

Bergemann, M., \& Gehren, T. 2008, A\&A, 492, 823

Bergemann, M., Pickering, J. C., \& Gehren, T. 2010, MNRAS, 401, 1334

Bessell, M. S., \& Christlieb, N. 2005, in IAU Symposium, Vol. 228, From Lithium to Uranium: Elemental Tracers of Early Cosmic Evolution, ed. V. Hill, P. François, \& F. Primas, 237-238

Bonifacio, P., Caffau, E., \& Ludwig, H.-G. 2009a,

Mem. Soc. Astron. Italiana, 80, 739

Bonifacio, P., Caffau, E., Venn, K. A., \& Lambert, D. L. 2012, A\&A, 544, A102

Bonifacio, P. et al. 2007, A\&A, 462, 851

. 2009b, A\&A, 501, 519

Bromm, V., \& Loeb, A. 2003, Nature, 425, 812

Bruenn, S. W. et al. 2013, ApJ, 767, L6

Burrows, A. 2013, Reviews of Modern Physics, 85, 245

Busso, M., Gallino, R., \& Wasserburg, G. J. 1999, ARA\&A, 37, 239

Caffau, E. et al. 2011a, Nature, 477, 67

- 2011b, A\&A, 534, A4

. 2012, A\&A, 542, A51

Campbell, S. W., Lugaro, M., \& Karakas, A. I. 2010, A\&A, 522, L6

Cayrel, R. et al. 2004, A\&A, 416, 1117

Chieffi, A., \& Limongi, M. 2012, ArXiv e-prints, 1212.2759

Christlieb, N. 2003, in Reviews in Modern Astronomy, Vol. 16 ,

Reviews in Modern Astronomy, ed. R. E. Schielicke, 191-+

Christlieb, N. et al. 2002, Nature, 419, 904

Christlieb, N., Gustafsson, B., Korn, A. J., Barklem, P. S., Beers, T. C., Bessell, M. S., Karlsson, T., \& Mizuno-Wiedner, M. 2004, ApJ, 603, 708

Clark, P. C., Glover, S. C. O., Smith, R. J., Greif, T. H., Klessen, R. S., \& Bromm, V. 2011, Science, 331, 1040

Coc, A., Vangioni-Flam, E., Descouvemont, P., Adahchour, A., \& Angulo, C. 2004, ApJ, 600, 544

Cohen, J. G., Christlieb, N., McWilliam, A., Shectman, S., Thompson, I., Melendez, J., Wisotzki, L., \& Reimers, D. 2008, ApJ, 672, 320

Cohen, J. G. et al. 2004, ApJ, 612, 1107

Cohen, J. G., McWilliam, A., Christlieb, N., Shectman, S., Thompson, I., Melendez, J., Wisotzki, L., \& Reimers, D. 2007, ApJ, 659, L161

Cohen, J. G. et al. 2006, AJ, 132, 137

Collet, R., Asplund, M., \& Trampedach, R. 2006, ApJ, 644, L121

Demorest, P. B., Pennucci, T., Ransom, S. M., Roberts, M. S. E. \& Hessels, J. W. T. 2010, Nature, 467, 1081

Depagne, E. et al. 2002, A\&A, 390, 187

Dopcke, G., Glover, S. C. O., Clark, P. C., \& Klessen, R. S. 2012 ArXiv e-prints, 1203.6842

Ekström, S., Meynet, G., Chiappini, C., Hirschi, R., \& Maeder, A. $2008, A \& A, 489,685$

Farr, W. M., Sravan, N., Cantrell, A., Kreidberg, L., Bailyn, C. D., Mandel, I., \& Kalogera, V. 2011, ApJ, 741, 103

Foley, R. J., Brown, P. J., Rest, A., Challis, P. J., Kirshner, R. P., \& Wood-Vasey, W. M. 2010, ApJ, 708, L61

Frebel, A. et al. 2005, Nature, 434, 871

Frebel, A., Collet, R., Eriksson, K., Christlieb, N., \& Aoki, W. 2008, ApJ, 684, 588

Frebel, A., Kirby, E. N., \& Simon, J. D. 2010, Nature, 464, 72

Frebel, A., Norris, J. E., Aoki, W., Honda, S., Bessell, M. S., Takada-Hidai, M., Beers, T. C., \& Christlieb, N. 2007, ApJ, 658,534

Fröhlich, C. et al. 2006a, ApJ, 637, 415

Fröhlich, C., Martínez-Pinedo, G., Liebendörfer, M., Thielemann, F., Bravo, E., Hix, W. R., Langanke, K., \& Zinner, N. T. 2006b, Physical Review Letters, 96, 142502

Fujimoto, M. Y., Ikeda, Y., \& Iben, Jr., I. 2000, ApJ, 529, L25

Fynbo, J. P. U. et al. 2006, Nature, 444, 1047

González Hernández, J. I. et al. 2008, A\&\&, 480, 233

González Hernández, J. I., Bonifacio, P., Ludwig, H.-G., Caffau,

E., Behara, N. T., \& Freytag, B. 2010, A\&A, 519, A46

Heger, A., \& Woosley, S. E. 2010, ApJ, 724, 341

Hix, W. R., \& Thielemann, F. 1996, ApJ, 460, 869 1999, ApJ, 511, 862

Honda, S., Aoki, W., Kajino, T., Ando, H., Beers, T. C. Izumiura, H., Sadakane, K., \& Takada-Hidai, M. 2004, ApJ, 607,474

Horiuchi, S., Beacom, J. F., Kochanek, C. S., Prieto, J. L., Stanek, K. Z., \& Thompson, T. A. 2011, ApJ, 738, 154

Ishigaki, M., Chiba, M., \& Aoki, W. 2010, PASJ, 62, 143
Ito, H., Aoki, W., Beers, T. C., Tominaga, N., Honda, S., \& Carollo, D. 2013, Ap.J, 698, L37

Ito, H., Aoki, W. Honda, S., \& Beers, T. C. 2009, ApJ, 698, L37

Iwamoto, N., Kajino, T., Mathews, G. J., Fujimoto, M. Y., \& Aoki, W. 2004, ApJ, 602, 377

Iwamoto, N., Umeda, H., Nomoto, K., Tominaga, N Thielemann, F., \& Hix, W. R. 2006, in American Institute of Physics Conference Series, Vol. 847, Origin of Matter and Evolution of Galaxies, ed. S. Kubono, W. Aoki, T. Kajino, T. Motobayashi, \& K. Nomoto, 409-411

Iwamoto, N., Umeda, H., Tominaga, N., Nomoto, K., \& Maeda, K. 2005, Science, 309, 451

Izutani, N., \& Umeda, H. 2010, ApJ, 720, L1

Janka, H.-T., Hanke, F., Huedepohl, L., Marek, A., Mueller, B., \& Obergaulinger, M. 2012, ArXiv e-prints, 1211.1378

Joggerst, C. C., Almgren, A., Bell, J., Heger, A., Whalen, D., \& Woosley, S. E. 2010, ApJ, 709, 11

Joggerst, C. C., Woosley, S. E., \& Heger, A. 2009, ApJ, 693, 1780

Johnson, J. A., \& Bolte, M. 2002, ApJ, 579, L87

Johnson, J. A., Herwig, F., Beers, T. C., \& Christlieb, N. 2007, ApJ, 658, 1203

Karakas, A. I. 2010, MNRAS, 403, 1413

Koch, A., McWilliam, A., Grebel, E. K., Zucker, D. B., \& Belokurov, V. 2008, ApJ, 688, L13

Komiya, Y., Suda, T., Minaguchi, H., Shigeyama, T., Aoki, W., \& Fujimoto, M. Y. 2007, ApJ, 658, 367

Kotake, K., Sumiyoshi, K., Yamada, S., Takiwaki, T., Kuroda, T., Suwa, Y., \& Nagakura, H. 2012, Progress of Theoretical and Experimental Physics, 2012, 301

Lai, D. K., Bolte, M., Johnson, J. A., Lucatello, S., Heger, A., \& Woosley, S. E. 2008, ApJ, 681, 1524

Li, W. et al. 2011, MNRAS, 412, 1441

Limongi, M., \& Chieffi, A. 2003, ApJ, 592, 404

Lind, K., Asplund, M., Barklem, P. S., \& Belyaev, A. K. 2011, A\&A, 528, A103

Lucatello, S., Tsangarides, S., Beers, T. C., Carretta, E., Gratton, R. G., \& Ryan, S. G. 2005, ApJ, 625, 825

Lugaro, M., Karakas, A. I., Stancliffe, R. J., \& Rijs, C. 2012, ApJ, 747, 2

Maeda, K. et al. 2008, Science, 319, 1220

Mashonkina, L., Korn, A. J., \& Przybilla, N. 2007, A\&A, 461, 261

Meakin, C. A., \& Arnett, D. 2007, ApJ, 667, 448

Meynet, G., Ekström, S., \& Maeder, A. 2006, A\&A, 447, 623

Moriya, T., Tominaga, N., Tanaka, M., Nomoto, K., Sauer, D. N., Mazzali, P. A., Maeda, K., \& Suzuki, T. 2010, ApJ, 719, 1445

Nakasato, N., \& Shigeyama, T. 2000, ApJ, 541, L59

Nishimura, T., Aikawa, M., Suda, T., \& Fujimoto, M. Y. 2009, PASJ, 61, 909

Nissen, P. E., Primas, F., Asplund, M., \& Lambert, D. L. 2002, A\&A, 390, 235

Nomoto, K. 1982, ApJ, 253, 798

Nomoto, K., \& Hashimoto, M. 1988, Phys. Rep., 163, 13

Nomoto, K., Kobayashi, C., \& Tominaga, N. 2013, ArXiv Astrophysics e-prints

Nomoto, K., Tominaga, N., Umeda, H., Kobayashi, C., \& Maeda, K. 2006, Nuclear Physics A, 777, 424

Norris, J. E., Christlieb, N., Bessell, M. S., Asplund, M., Eriksson, K., \& Korn, A. J. 2012, ApJ, 753, 150

Norris, J. E., Christlieb, N., Korn, A. J., Eriksson, K., Bessell, M. S., Beers, T. C., Wisotzki, L., \& Reimers, D. 2007, ApJ, 670,774

Norris, J. E., Gilmore, G., Wyse, R. F. G., Yong, D., \& Frebel, A. 2010a, ApJ, 722, L104

Norris, J. E., Ryan, S. G., \& Beers, T. C. 2001, ApJ, 561, 1034

Norris, J. E., Ryan, S. G., Beers, T. C., Aoki, W., \& Ando, H. 2002, ApJ, 569, L107

Norris, J. E. et al. 2013, ApJ, 762, 28

Norris, J. E., Yong, D., Gilmore, G., \& Wyse, R. F. G. 2010b, ApJ, 711, 350

Omukai, K., Tsuribe, T., Schneider, R., \& Ferrara, A. 2005, ApJ, 626, 627

Özel, F., Psaltis, D., Narayan, R., \& McClintock, J. E. 2010, ApJ, 725,1918

Plez, B., \& Cohen, J. G. 2005, A\&A, 434, 1117

Pols, O. R., Izzard, R. G., Stancliffe, R. J., \& Glebbeek, E. 2012, A\&A, 547, A76

Pruet, J., Surman, R., \& McLaughlin, G. C. 2004, ApJ, 602, L101

Pruet, J., Woosley, S. E., Buras, R., Janka, H., \& Hoffman, R. D. 2005, ApJ, 623, 325

Richardson, D., Branch, D., Casebeer, D., Millard, J., Thomas, R. C., \& Baron, E. 2002, AJ, 123, 745

Ritter, J. S., Safranek-Shrader, C., Gnat, O., Milosavljevic, M., \& Bromm, V. 2012, ArXiv e-prints, 1203.2957

Ryan, S. G., Norris, J. E., \& Beers, T. C. 1996, ApJ, 471, 254

Schneider, R., Omukai, K., Inoue, A. K., \& Ferrara, A. 2006 MNRAS, 369, 1437

Shigeyama, T., \& Tsujimoto, T. 1998, ApJ, 507, L135 
TABLE 1

THE PROGENITOR MODELS

\begin{tabular}{ccccc}
\hline \hline Model name & $\begin{array}{c}M_{\mathrm{MS}} \\
{\left[\mathrm{M}_{\odot}\right]}\end{array}$ & Mixing enhancement & Figure & Reference \\
\hline $\mathrm{A}$ & 25 & No & Fig. 1 $1 \mathrm{l}$ & Iwamoto et al. (2005) \\
$\mathrm{B}$ & 25 & Yes & Fig. 10 & Iwamoto et al. (2005) \\
$\mathrm{C}$ & 40 & No & Fig. 1. & Tominaga et al. (2007a) \\
\hline
\end{tabular}

Smith, V. V., Cunha, K., Ivans, I. I., Lattanzio, J. C., Campbell, S., \& Hinkle, K. H. 2005, ApJ, 633, 392

Spite, M. et al. 2012, A\&A, 541, A143

. 2011, A\&A, 528, A9

2006, A\&A, 455, 291

2005, A\&A, 430, 655

Stancliffe, R. J. 2009, MNRAS, 394, 1051

Starkenburg, E. et al. 2012, ArXiv e-prints, 1211.4592

Stephens, A., \& Boesgaard, A. M. 2002, AJ, 123, 1647

Stephenson, F. R., \& Green, D. A. 2002, Historical supernovae and their remnants, by F. Richard Stephenson and David

A. Green. International series in astronomy and astrophysics, vol. 5. Oxford: Clarendon Press, 2002, ISBN 0198507666, 5

Suda, T., Aikawa, M., Machida, M. N., Fujimoto, M. Y., \& Iben, Jr., I. 2004, ApJ, 611, 476

Suda, T., \& Fujimoto, M. Y. 2010, MNRAS, 405, 177

Suda, T. et al. 2008, PASJ, 60, 1159

Suda, T., Yamada, S., Katsuta, Y., Komiya, Y., Ishizuka, C., Aoki, W., \& Fujimoto, M. Y. 2011, MNRAS, 412, 843

Susa, H. 2013, ArXiv e-prints, 1304.6794

Tafelmeyer, M. et al. 2010, A\&A, 524, A58

Takeda, Y., Kaneko, H., Matsumoto, N., Oshino, S., Ito, H., \& Shibuya, T. 2009, PASJ, 61, 563

Takeda, Y., Zhao, G., Chen, Y.-Q., Qiu, H.-M., \& Takada-Hidai, M. 2002, PASJ, 54, 275

Tanaka, M. et al. 2012, ApJ, 754, 63
- 2009, ApJ, 692, 1131

Thornton, K., Gaudlitz, M., Janka, H., \& Steinmetz, M. 1998, ApJ, 500, 95

Tominaga, N. 2009, ApJ, 690, 526

Tominaga, N., Maeda, K., Umeda, H., Nomoto, K., Tanaka, M.,

Iwamoto, N., Suzuki, T., \& Mazzali, P. A. 2007a, ApJ, 657, L77

Tominaga, N., Umeda, H., \& Nomoto, K. 2007b, ApJ, 660, 516

Tumlinson, J. 2006, ApJ, 641, 1

Umeda, H., \& Nomoto, K. 2002, ApJ, 565, 385 2003, Nature, 422, 871

- 2005, ApJ, 619, 427

Valenti, S. et al. 2009, Nature, 459, 674

Venn, K. A. et al. 2012, ApJ, 751, 102

Wiescher, M., Görres, J., Uberseder, E., Imbriani, G., \& Pignatari, M. 2010, Annual Review of Nuclear and Particle Science, 60, 381

Yanny, B. et al. 2009, AJ, 137, 4377

Yong, D. et al. 2013, ApJ, 762, 26

Yoshii, Y. 1981, A\&A, 97, 280

Zampieri, L. Pastorello, A., Turatto, M., Cappellaro, E., Benetti, S., Altavilla, G., Mazzali, P., \& Hamuy, M. 2003, MNRAS, 338, 711

Zhang, H. W., Gehren, T., Butler, K., Shi, J. R., \& Zhao, G. 2006, A\&A, 457, 645 
TABLE 2

EMP STARS WITH $[\mathrm{FE} / \mathrm{H}] \lesssim-3.5$

\begin{tabular}{|c|c|c|}
\hline Star name & {$[\mathrm{Fe} / \mathrm{H}]$} & Figure: (color) reference \\
\hline \multicolumn{3}{|r|}{ EMP stars } \\
\hline HE 0146-1548 & -3.46 & Fig. 2 h: (red) 1,2 \\
\hline CS 22189-009 & -3.48 & Fig. 2 b: (red) $3,4,5,6,7,8,9$, (blue) 1 \\
\hline SDSS J090733+024608 & -3.50 & Fig. 2 : (red) 10 \\
\hline CS 22897-008 & -3.50 & Fig. 2 d: (red) $3,4,5,6,7,8,9$, (blue) 1 \\
\hline BS $16545-089$ & -3.50 & Fig. 2 : (red) 11, (blue) 12 , (green) 13, (magenta) 1 \\
\hline CS 22963-004 & -3.54 & Fig. 2f: (red) 13 , (blue) 1 \\
\hline CS 22968-014 & -3.58 & Fig. 2 : : (red) $3,4,5,6,7,8,9$, (blue) 1 \\
\hline HE $1356-0622$ & -3.58 & Fig. 2h: (red) 14, (blue) 1 \\
\hline HE $1347-1025$ & -3.62 & Fig. 2 : (red) 14, (blue) 1 \\
\hline BS 16076-006 & -3.66 & Fig. 2 : (red) 15, (blue) 1 \\
\hline CS $22876-032 \mathrm{AB}$ & -3.66 & Fig. 31: (red) 16 (Star A), (blue) 16 (Star B) \\
\hline HE 0049-3948 & -3.68 & Fig. 3 p: (red) 1 \\
\hline HE $1320-2952$ & -3.69 & Fig. 3 : $(r e d) 1$ \\
\hline BS $16467-062$ & -3.74 & Fig. 31: (red) $3,4,5,6,7,8,9$, (blue) 14, (green) 13, (magenta) 1 \\
\hline CS 22172-002 & -3.74 & Fig. 3e: (red) $3,4,5,9$, (blue) 17 , (green) 1 \\
\hline HE 0228-4047 & -3.75 & Fig. 3 : (red) 1 (dwarf), (blue) 1 (subgiant) \\
\hline G 238-30 & -3.77 & Fig. $\overline{3}$ : (red) 18, (blue) 19 \\
\hline CS 22885-096 & -3.77 & Fig. 3 h: (red) $3,4,5,6,7,8,9$, (blue) 17 , (green) 1 \\
\hline \multicolumn{3}{|r|}{ CEMP stars } \\
\hline HE 1150-0428 & -3.47 & Fig. 4 h: (red) 20, (blue) 1 \\
\hline HE $1012-1540$ & -3.48 & Fig. $4 \mathrm{~b}$ : (red) 14, (blue) 1 \\
\hline CS 29498-043 & -3.52 & Fig. 4 : (red) 21, (blue) 1 \\
\hline HE 1506-0113 & -3.54 & Fig. 4 d: $($ red $) 1$ \\
\hline CS 29527-015 & -3.55 & Fig. 4 : (red) $6,7,8,9$, (blue) 1 \\
\hline HE $0132-2429$ & -3.70 & Fig. 崩: (red) 14, (blue) 1 \\
\hline HE $1300+0157$ & -3.76 & Fig. 4 : (red) 22 (subgiant), (blue) 22 (dwarf), (green) 23, (magenta) 14, (yellow) 1 \\
\hline $\mathrm{BD}+44^{\circ} 493$ & -3.78 & Fig. 4 h: (red) $24,($ blue $) 25$ \\
\hline HE $1201-1512$ & -3.89 & Fig. 4 : (red) 1 (dwarf), (blue) 1 (subgiant) \\
\hline HE 2139-5432 & -4.02 & Fig. 4 : (red) 1 \\
\hline $53327-2044-515$ & -4.04 & Fig. 4 k: (red) 1,2 (dwarf), (blue) 1,2 (subgiant) \\
\hline G 77-61 & -4.08 & Fig. 4]: (red) 26,27 \\
\hline \multicolumn{3}{|r|}{ NEMP stars } \\
\hline CS 22952-015 & -3.44 & Fig. 51 h: (red) $3,4,5,6,7,8,9$, (blue) 28, (green) 1 \\
\hline BS $16550-087$ & -3.54 & Fig. 5 b: (red) 13 , (blue) 1 \\
\hline CS 22949-037 & -3.89 & Fig. 5F: (red) $3,4,5,6,7,8,9,29$, (blue) 17,30, (green) 14, (magenta) 1 \\
\hline CS 30336-049 & -4.07 & Fig. $5 \mathrm{~d}$ : (red) 13, (blue) 1 \\
\hline HE $0057-5959$ & -4.08 & Fig. 5 : (red) 1,2 \\
\hline CD-38:245 & -4.11 & Fig. 亗: (red) $3,4,5,6,7,8,9$, (blue) 17 , (green) 1 \\
\hline \multicolumn{3}{|r|}{ UMP stars } \\
\hline HE $0557-4840$ & -4.77 & Fig. 6 h: $($ red $) 31,32\left(T_{\text {eff }}=4900 \mathrm{~K}\right),($ blue $) 31\left(T_{\text {eff }}=5100 \mathrm{~K}\right)($ green $) 1,2$ \\
\hline SDSS J102915+172927 & -4.92 & Fig. 6 b: (red) 33,34 \\
\hline \multicolumn{3}{|r|}{ HMP stars } \\
\hline HE 0107-5240 & -5.61 & Fig. 7h: (red) 35,36, (blue) 37, (green) 1,2 \\
\hline HE $1327-2326$ & -5.88 & Fig. $7 \mathrm{~b}:$ (red) 38,39, (blue) 38 , (green) 1,2 \\
\hline \multicolumn{3}{|r|}{ EMP stars in dwarf galaxies } \\
\hline Scl $031-11$ & -3.47 & Fig. 81: (red) 40 \\
\hline Scl $07-49$ & -3.48 & Fig. $8 \mathrm{p}:($ red) 41 \\
\hline SEGUE1-7 & -3.57 & Fig. 81: (red) 42 \\
\hline Fnx $05-42$ & -3.66 & Fig. 8d: $(r e d) 41$ \\
\hline Boo-1137 & -3.71 & Fig. 8p: (red) 43 \\
\hline S 1020549 & -3.81 & Fig. 8 : (red) 44 \\
\hline Scl $07-50$ & -3.96 & Fig. 8 g: $(r e d) 41$ \\
\hline \multicolumn{3}{|r|}{ MP stars in Hercules dwarf spheroidal galaxy } \\
\hline Her-2 & -2.02 & Fig. 12 (red) 45 \\
\hline Her-3 & -2.04 & Fig. 12 (blue) 45 \\
\hline
\end{tabular}

References. - (1) Yong et al. (2013), (2) Norris et al. (2013), (3) Cavrel et al. (2004), (4) Spite et al. (2005), (5) Spite et al. (2006), (6) Andrievsky et al. (2007), (7) Andrievsky et al. (2008), (8) Andrievsky et al. (2010), (9) Spite et al. (2012), (10) Caffau et al. (2011b), (11) Cohen et al. (2004), (12) Aoki et al. (2009), (13) Lai et al. (2008), (14) Cohen et al. (2008), (15) Bonifacio et al. (2009b), (16) González Hernández et al. (2008), (17) Norris et al. (2001), (18) Ishigaki et al. (2010), (19) Stephens \& Boesgaard (2002), (20) Cohen et al. (2006), (21) Aoki et al. (2004), (22) Frebel et al. (2007), (23) Barklem et al. (2005), (24) Ito et al. (2009), (25) Ito et al. (2013), (26) Plez \& Cohen (2005), (27) Beers et al. (2007), (28) Honda et al (2004), (29) Spite et al. (2011) (30) Norris et al. (2002), (31) Norris et al. (2007), (32) Norris et al. (2012), (33) Caffau et al. (2011a), (34) Caffau et al. (2012), (35) Christlieb et al. (2004), (36) Bessell \& Christlieb (2005), (37) Collet et al. (2006), (38) Frebel et al. (2008), (39) Bonifacio et al. (2012), (40) Starkenburg et al. (2012), (41) Tafelmever et al. (2010), (42) Norris et al. (2010a), (43) Norris et al. (2010b), (44) Frebel et al. (2010), (45) Koch et al. (2008)

Note. - The columns show the name of star, the metallicity $[\mathrm{Fe} / \mathrm{H}]$, and the figure number with references and color legends. 
TABLE 3

POP III SN MODELS WITH $25 \mathrm{M}_{\odot}$ PROGENITOR

\begin{tabular}{|c|c|c|c|c|c|c|c|c|c|c|c|}
\hline Star name & $E_{51}$ & $M_{\mathrm{ej}}(\mathrm{Fe}) / \mathrm{M}_{\odot}$ & $M_{\mathrm{rem}} / \mathrm{M}_{\odot}$ & $M_{\text {cut }} / \mathrm{M}_{\odot}$ & $M_{\operatorname{mix}} / \mathrm{M}_{\odot}$ & $\overline{f_{\mathrm{ej}}}$ & $\overline{f_{\rho}}$ & $Y_{\mathrm{e}}$ modification & Progenitor & Figure (line type) & Distribution \\
\hline \multicolumn{12}{|c|}{ EMP stars } \\
\hline HE $0146-1548$ & 5 & $2.38 \times 10^{-2}$ & 3.45 & 1.78 & 3.75 & 0.15 & $1 / 8$ & Mn \& Co & $\mathrm{A}$ & Fig. $[2$ a (solid) & Yes \\
\hline CS 22189-009 & 20 & $7.76 \times 10^{-2}$ & 4.20 & 1.72 & 4.51 & 0.11 & $1 / 2$ & $\mathrm{Mn} \& \mathrm{Co}$ & $\mathrm{B}$ & Fig. $2 \mathrm{~b}$ (solid) & Yes \\
\hline SDSS J090733+024608 & 5 & $1.85 \times 10^{-1}$ & 1.74 & 1.74 & - & 1.0 & $1 / 8$ & No & $\mathrm{A}$ & Fig. 2 (solid) & \\
\hline CS 22897-008 & 20 & $5.65 \times 10^{-2}$ & 4.22 & 1.72 & 4.43 & 0.08 & $1 / 2$ & $\mathrm{Mn} \& \mathrm{Co}$ & $\mathrm{B}$ & Fig. $2 \mathrm{~d}$ (solid) & Yes \\
\hline - & 5 & $8.46 \times 10^{-2}$ & 2.58 & 1.61 & 2.99 & 0.3 & $1 / 6$ & $\mathrm{Mn} \& \mathrm{Co}$ & B & Fig. 21 (dashed) & \\
\hline BS 16545-089 & 20 & $1.07 \times 10^{-1}$ & 4.02 & 1.74 & 4.59 & 0.2 & $1 / 3$ & $\mathrm{Mn} \& \mathrm{Co}$ & A & Fig. 2 (solid) & \\
\hline CS 22963-004 & 5 & $2.85 \times 10^{-2}$ & 4.75 & 1.77 & 5.28 & 0.15 & $1 / 6$ & $\mathrm{Mn} \& \mathrm{Co}$ & B & Fig. [2] (solid) & Yes \\
\hline CS 22968-014 & 20 & $8.61 \times 10^{-2}$ & 3.84 & 1.70 & 4.14 & 0.12 & $1 / 2$ & $\mathrm{Mn} \& \mathrm{Co}$ & $\mathrm{B}$ & Fig. 2 p (solid) & Yes \\
\hline HE $1356-0622$ & 5 & $1.11 \times 10^{-1}$ & 1.96 & 1.78 & 2.31 & 0.65 & $1 / 8$ & No & A & Fig. 2h (solid) & \\
\hline HE $1347-1025$ & 5 & $9.85 \times 10^{-2}$ & 4.00 & 1.72 & 6.27 & 0.5 & $1 / 8$ & $\mathrm{Co}$ & A & Fig. 2 (solid) & Yes \\
\hline BS 16076-006 & 5 & $5.10 \times 10^{-2}$ & 3.87 & 1.70 & 4.59 & 0.25 & $1 / 8$ & $\mathrm{Co}$ & B & Fig. 2 (solid) & Yes \\
\hline CS 22876-032AB & 20 & $4.24 \times 10^{-2}$ & 4.49 & 1.72 & 4.67 & 0.06 & $1 / 2$ & $\mathrm{Mn} \& \mathrm{Co}$ & A & Fig. $[3$ a (solid) & Yes \\
\hline HE 0049-3948 & 20 & $8.34 \times 10^{-2}$ & 4.28 & 1.66 & 4.74 & 0.15 & $1 / 3$ & No & $\mathrm{A}$ & Fig. 3 b (solid) & \\
\hline HE $1320-2952$ & 10 & $1.30 \times 10^{-1}$ & 2.73 & 2.00 & 3.22 & 0.4 & $1 / 4$ & $\mathrm{Co}$ & A & Fig. 3 (solid) & \\
\hline BS 16467-062 & 20 & $4.36 \times 10^{-2}$ & 4.51 & 2.25 & 4.81 & 0.12 & $1 / 3$ & $\mathrm{Mn} \& \mathrm{Co}$ & A & Fig. $3 \mathrm{~d}$ (solid) & Yes \\
\hline - & 5 & $7.44 \times 10^{-2}$ & 3.79 & 1.86 & 5.72 & 0.5 & $1 / 6$ & $\mathrm{Mn} \& \mathrm{Co}$ & A & Fig. 31 (dashed) & \\
\hline CS 22172-002 & 20 & $7.76 \times 10^{-2}$ & 3.87 & 1.72 & 4.14 & 0.11 & $1 / 2$ & $\mathrm{Mn} \& \mathrm{Co}$ & B & Fig. 31 (solid) & Yes \\
\hline HE $0228-4047$ & 20 & $1.36 \times 10^{-1}$ & 3.27 & 1.63 & 3.98 & 0.3 & $1 / 4$ & No & A & Fig. 何 (solid) & \\
\hline G 238-30 & 5 & $6.80 \times 10^{-2}$ & 2.55 & 1.78 & 3.07 & 0.4 & $1 / 8$ & No & A & Fig. 35 (solid) & \\
\hline CS 22885-096 & 20 & $4.95 \times 10^{-2}$ & 3.76 & 2.12 & 3.98 & 0.12 & $1 / 3$ & Co & B & Fig. 3n (solid) & Yes \\
\hline \multicolumn{12}{|c|}{ CEMP stars } \\
\hline HE $1150-0428$ & 5 & $3.43 \times 10^{-4}$ & 5.83 & 1.67 & 5.84 & 0.003 & $1 / 16$ & No & $\mathrm{B}$ & Fig. 4a (solid) & \\
\hline - & 5 & $3.51 \times 10^{-4}$ & 5.83 & 1.67 & 5.84 & 0.003 & $1 / 16$ & No & A & Fig. 4 (dashed) & \\
\hline HE $1012-1540$ & 20 & $1.11 \times 10^{-3}$ & 4.81 & 2.25 & 4.81 & 0.003 & $1 / 3$ & $\mathrm{Co}$ & A & Fig. $4 \mathrm{~b}$ (solid) & Yes \\
\hline CS 29498-043 & 20 & $9.08 \times 10^{-4}$ & 5.11 & 1.90 & 5.12 & 0.0015 & $1 / 2$ & No & B & Fig. $\overline{4}$ (solid) & Yes \\
\hline - & 20 & $9.10 \times 10^{-4}$ & 5.27 & 1.90 & 5.28 & 0.0015 & $1 / 2$ & No & A & Fig. 座 (dashed) & \\
\hline HE $1506-0113$ & 20 & $6.80 \times 10^{-3}$ & 5.15 & 1.86 & 5.20 & 0.015 & $1 / 4$ & $\mathrm{Mn} \& \mathrm{Co}$ & $\mathrm{A}$ & Fig. 4d (solid) & Yes \\
\hline CS 29527-015 & 20 & $6.52 \times 10^{-3}$ & 5.14 & 1.76 & 5.20 & 0.015 & $1 / 4$ & $\mathrm{Mn} \& \mathrm{Co}$ & A & Fig. 4 (solid) & Yes \\
\hline HE $0132-2429$ & 20 & $1.24 \times 10^{-2}$ & 5.39 & 1.81 & 5.50 & 0.03 & $1 / 4$ & $\mathrm{Mn} \& \mathrm{Co}$ & $\mathrm{B}$ & Fig. 岸 (solid) & Yes \\
\hline HE $1300+0157$ & 20 & $5.21 \times 10^{-2}$ & 3.25 & 1.76 & 3.46 & 0.12 & $1 / 4$ & $\mathrm{Mn} \& \mathrm{Co}$ & A & Fig. 4, (solid) & Yes \\
\hline- & 20 & $3.42 \times 10^{-1}$ & 2.16 & 2.16 & - & 1.0 & $1 / 4$ & $\mathrm{Mn} \& \mathrm{Co}$ & $\mathrm{A}$ & Fig. $4 \vec{b}$ (dashed) & \\
\hline - & 20 & $3.58 \times 10^{-3}$ & 5.57 & 2.08 & 5.61 & 0.01 & $1 / 4$ & $\mathrm{Mn} \& \mathrm{Co}$ & A & Fig. 4 (dotted) & \\
\hline $\mathrm{BD}+44^{\circ} 493$ & 5 & $6.17 \times 10^{-3}$ & 5.33 & 1.90 & 5.50 & 0.045 & $1 / 8$ & Co & $\mathrm{A}$ & Fig. 4h (solid) & Yes \\
\hline HE $1201-1512$ & 5 & $9.17 \times 10^{-3}$ & 5.65 & 1.55 & 5.80 & 0.035 & $1 / 8$ & $\mathrm{Mn} \& \mathrm{Co}$ & $\mathrm{A}$ & Fig. 4. (solid) & Yes \\
\hline - & 5 & $4.53 \times 10^{-3}$ & 5.72 & 1.78 & 5.80 & 0.02 & $1 / 4$ & $\mathrm{Mn} \& \mathrm{Co}$ & A & Fig. 4 (solid) & \\
\hline HE $2139-5432$ & 5 & $6.78 \times 10^{-4}$ & 5.03 & 1.62 & 5.04 & 0.003 & $1 / 8$ & Mn \& Co & $\mathrm{B}$ & Fig. [4] (solid) & Yes \\
\hline 53327-2044-515 & 5 & $8.51 \times 10^{-3}$ & 5.56 & 1.76 & 5.76 & 0.05 & $1 / 8$ & $\mathrm{Mn} \& \mathrm{Co}$ & A & Fig. 4 (solid) & Yes \\
\hline - & 5 & $3.26 \times 10^{-3}$ & 5.66 & 1.74 & 5.72 & 0.015 & $1 / 6$ & $\mathrm{Mn} \& \mathrm{Co}$ & $\mathrm{A}$ & Fig. 4 k (dashed) & \\
\hline G $77-61$ & 1 & $1.85 \times 10^{-4}$ & 6.17 & 1.90 & 6.18 & 0.002 & No & $\mathrm{Mn} \& \mathrm{Co}$ & $\mathrm{B}$ & Fig. 4 (solid) & \\
\hline \multicolumn{12}{|c|}{ NEMP stars } \\
\hline CS 22952-015 & 5 & $3.09 \times 10^{-2}$ & 4.46 & 1.84 & 5.12 & 0.2 & $1 / 6$ & $\mathrm{Mn}$ & $\mathrm{A}$ & Fig. 5ha (solid) & Yes \\
\hline BS $16550-087$ & 10 & $7.68 \times 10^{-2}$ & 3.14 & 1.79 & 3.46 & 0.19 & $1 / 4$ & $\mathrm{Mn}$ & A & Fig. $\overline{50}$ (solid) & Yes \\
\hline CS 22949-037 & 10 & $2.42 \times 10^{-3}$ & 4.34 & 1.66 & 4.36 & 0.005 & $1 / 4$ & $\mathrm{Mn} \& \mathrm{Co}$ & A & Fig. & Yes \\
\hline - & 10 & $4.85 \times 10^{-3}$ & 4.11 & 1.66 & 4.13 & 0.01 & $1 / 4$ & $\mathrm{Mn} \& \mathrm{Co}$ & A & Fig. 5 (dashed) & \\
\hline CS 30336-049 & 5 & $2.54 \times 10^{-2}$ & 5.01 & 1.61 & 5.35 & 0.09 & $1 / 6$ & $\mathrm{Mn} \& \mathrm{Co}$ & $\mathrm{A}$ & Fig. [5] (solid) & Yes \\
\hline - & 5 & $1.69 \times 10^{-1}$ & 2.16 & 1.61 & 2.99 & 0.6 & $1 / 6$ & $\mathrm{Mn} \& \mathrm{Co}$ & A & Fig. 5 (dashed) & \\
\hline HE 0057-5959 & 5 & $6.80 \times 10^{-3}$ & 5.38 & 1.78 & 5.53 & 0.04 & $1 / 8$ & No & $\mathrm{A}$ & Fig. 5 (solid) & \\
\hline CD-38:245 & 20 & $6.10 \times 10^{-2}$ & 4.07 & 1.64 & 4.28 & 0.08 & $1 / 2$ & $\mathrm{Mn} \& \mathrm{Co}$ & A & Fig. 扂 (solid) & Yes \\
\hline \multicolumn{12}{|c|}{ UMP stars } \\
\hline HE 0557-4840 & 5 & $6.80 \times 10^{-3}$ & 5.57 & 1.78 & 5.72 & 0.04 & $1 / 8$ & No & $\mathrm{A}$ & Fig. 6a (solid) & Yes \\
\hline
\end{tabular}


TABLE 3 - Continued

\begin{tabular}{|c|c|c|c|c|c|c|c|c|c|c|c|}
\hline Star name & $E_{51}$ & $M_{\mathrm{ej}}(\mathrm{Fe}) / \mathrm{M}_{\odot}$ & $M_{\mathrm{rem}} / \mathrm{M}_{\odot}$ & $M_{\text {cut }} / \mathrm{M}_{\odot}$ & $M_{\text {mix }} / \mathrm{M}_{\odot}$ & $f_{\mathrm{ej}}$ & $f_{\rho}$ & $Y_{\mathrm{e}}$ modification & Progenitor & Figure (line type) & Distribution \\
\hline SDSS J102915+172927 & 20 & $1.21 \times 10^{-1}$ & 3.03 & 1.58 & 3.29 & 0.15 & $1 / 2$ & $\mathrm{Mn} \& \mathrm{Co}$ & A & Fig. 6p (solid) & \\
\hline - & 5 & $9.25 \times 10^{-2}$ & 3.49 & 1.78 & 5.20 & 0.5 & $1 / 6$ & $\mathrm{Mn} \& \mathrm{Co}$ & $\mathrm{A}$ & Fig. 6b (dashed) & \\
\hline \multicolumn{12}{|c|}{ HMP stars } \\
\hline HE $0107-5240$ & 5 & $8.02 \times 10^{-5}$ & 6.24 & 1.90 & 6.24 & 0.0005 & $1 / 4$ & $\mathrm{Mn} \& \mathrm{Co}$ & $\bar{A}$ & Fig. 17 (solid) & Yes \\
\hline HE $1327-2326$ & 5 & $1.45 \times 10^{-5}$ & 5.72 & 1.74 & 5.72 & 0.00008 & $1 / 8$ & No & B & Fig. 7 b (solid) & Yes \\
\hline- & 0.72 & $1.53 \times 10^{-5}$ & 5.72 & 1.69 & 5.72 & 0.00006 & $1 / 8$ & No & $\mathrm{B}$ & Fig. 7b (dashed) & \\
\hline \multicolumn{12}{|c|}{ EMP stars in dwarf galaxies } \\
\hline Scl 031-11 & 20 & $2.82 \times 10^{-1}$ & 3.26 & 1.72 & 4.28 & 0.4 & $1 / 2$ & $\mathrm{Mn} \& \mathrm{Co}$ & $\mathrm{A}$ & Fig. 8 a (solid) & \\
\hline - & 10 & $1.34 \times 10^{-2}$ & 5.80 & 1.80 & 5.92 & 0.03 & $1 / 3$ & $\mathrm{Mn} \& \mathrm{Co}$ & A & Fig. 81 (dashed) & \\
\hline Scl 07-49 & 20 & $1.77 \times 10^{-1}$ & 2.63 & 1.74 & 3.22 & 0.4 & $1 / 4$ & No & A & Fig. $8 \mathrm{~b}$ (solid) & \\
\hline SEGUE1-7 & 20 & $7.55 \times 10^{-4}$ & 5.72 & 2.53 & 5.72 & 0.003 & $1 / 4$ & Co & A & Fig. 8 (solid) & Yes \\
\hline - & 20 & $6.99 \times 10^{-4}$ & 5.72 & 1.96 & 5.72 & 0.0018 & $1 / 4$ & Co & A & Fig. 87 (dashed) & \\
\hline Fnx $05-42$ & 20 & $1.18 \times 10^{-1}$ & 3.36 & 2.20 & 3.98 & 0.35 & $1 / 4$ & No & A & Fig. $8 \mathrm{~d}$ (solid) & Yes \\
\hline Boo-1137 & 5 & $4.87 \times 10^{-2}$ & 4.65 & 1.90 & 5.72 & 0.28 & $1 / 4$ & Co & A & Fig. 87 (solid) & Yes \\
\hline - & 20 & $8.41 \times 10^{-2}$ & 3.30 & 1.94 & 3.60 & 0.18 & $1 / 3$ & Co & A & Fig. 8p (dashed) & \\
\hline S 1020549 & 10 & $1.43 \times 10^{-1}$ & 2.39 & 1.94 & 2.69 & 0.4 & $1 / 4$ & $\mathrm{Mn} \& \mathrm{Co}$ & A & Fig. 8f: (solid) & Yes \\
\hline Scl $07-50$ & 10 & $6.96 \times 10^{-2}$ & 4.75 & 1.94 & 5.46 & 0.2 & $1 / 4$ & $\mathrm{Co}$ & A & Fig. 8, (solid) & Yes \\
\hline \multicolumn{12}{|c|}{ MP stars in Hercules dwarf spheroidal galaxy } \\
\hline Her-2 & 20 & $1.41 \times 10^{-2}$ & 4.97 & 1.72 & 5.04 & 0.02 & $1 / 2$ & $\mathrm{Mn} \& \mathrm{Co}$ & $\bar{A}$ & Fig. 12 (solid) & \\
\hline Her-3 & 20 & $2.68 \times 10^{-2}$ & 4.59 & 1.74 & 4.74 & 0.05 & $1 / 3$ & $\mathrm{Mn} \& \mathrm{Co}$ & A & Fig. 12 (dashed) & \\
\hline
\end{tabular}

Nоте. - The numbers shown are the explosion energy, the ejected Fe mass, the final central remnant mass, the mass of inner boundary of the mixing region, the mass of outer boundary of the mixing region, the ejection factor, the "low-density" factor, the $Y_{\mathrm{e}}$ modification, the progenitor model, the figure number with line legend, and whether the model is used for the distribution in $\S$ The masses are in unit of $\mathrm{M}_{\odot}$. 
TABLE 4

POP III SN MODELS WITH $40 \mathrm{M}_{\odot}$ PROGENITOR

\begin{tabular}{|c|c|c|c|c|c|c|c|c|c|}
\hline Star name & $E_{51}$ & $M_{\mathrm{ej}}(\mathrm{Fe}) / \mathrm{M}_{\odot}$ & $M_{\mathrm{rem}} / \mathrm{M}_{\odot}$ & $M_{\text {cut }} / \mathrm{M}_{\odot}$ & $M_{\operatorname{mix}} / \mathrm{M}_{\odot}$ & $f_{\mathrm{ej}}$ & $f_{\rho}$ & $Y_{\mathrm{e}}$ modification & Figure (line type) \\
\hline HE $1347-1025$ & 20 & $1.44 \times 10^{-1}$ & 5.33 & 2.24 & 5.88 & 0.15 & $1 / 5$ & Mn \& Co & Fig. 9a (solid) \\
\hline CS 29527-015 & 30 & $1.88 \times 10^{-2}$ & 9.53 & 2.24 & 9.65 & 0.015 & $1 / 4$ & $\mathrm{Mn} \& \mathrm{Co}$ & Fig. $9 \mathrm{9}$ (solid) \\
\hline $\mathrm{BD}+44^{\circ} 493$ & 15 & $8.39 \times 10^{-3}$ & 12.23 & 2.47 & 12.38 & 0.015 & $1 / 8$ & $\mathrm{Co}$ & Fig. 97 (solid) \\
\hline- & 15 & $8.66 \times 10^{-3}$ & 12.23 & 2.84 & 12.38 & 0.02 & $1 / 8$ & $\mathrm{Co}$ & Fig. 9. (dashed) \\
\hline HE $0557-4840$ & 20 & $1.14 \times 10^{-2}$ & 12.49 & 3.19 & 12.68 & 0.02 & $1 / 5$ & No & Fig. 99 (solid) \\
\hline HE $0107-5240$ & 10 & $1.06 \times 10^{-4}$ & 13.89 & 2.03 & 13.89 & 0.0003 & $1 / 8$ & $\mathrm{Mn} \& \mathrm{Co}$ & Fig. 9 (solid) \\
\hline
\end{tabular}

Noте. - The numbers shown are the explosion energy, the ejected Fe mass, the final central remnant mass, the mass of inner boundary of the mixing region, the mass of outer boundary of the mixing region, the ejection factor, the "low-density" factor, the $Y_{\mathrm{e}}$ modification, and the figure number with line legend. The masses are in unit of $\mathrm{M}_{\odot}$. 